Pure and Applied Mathematics Quarterly

Volume 5, Number 1

(Special Issue: In honor of

Jean-Pierre Serre, Part 2 of 2)

$163-212,2009$

\title{
On Uniqueness of $p$-adic Period Morphisms
}

\author{
Wiesława Nizioł
}

\begin{abstract}
We present uniqueness criteria for $p$-adic period morphisms for proper varieties and show that they imply equality of the $p$-adic period morphisms defined using the syntomic, almost étale and motivic constructions. Keywords: p-adic Hodge theory, comparison morphisms, motivic cohomology
\end{abstract}

\section{Contents}

1. Introduction 164

2. Preliminaries 166

2.1. Rings of periods 166

$\begin{array}{lll}\text { 2.2. Syntomic cohomology } & 171\end{array}$

2.3. Chern classes 176

$\begin{array}{lll}2.4 . & p \text {-adic comparison theorems } & 179\end{array}$

3. Motivic period morphisms and uniqueness criteria 184

4. Syntomic period morphisms 189

4.1. Compatibility with higher Chern classes 194

5. Faltings period morphisms 198

5.1. Compatibility with higher Chern classes 203

Received July 9, 2006.

This research was supported in part by the NSF grant DMS0402913. 
6. Comparison of $p$-adic period morphisms

\section{INTRODUCTION}

We present uniqueness criteria for $p$-adic period morphisms for proper varieties and show that they imply equality of the $p$-adic period morphisms defined using the syntomic, almost étale and motivic constructions. As an illustration, consider the simplest case: good reduction with rational coefficients. We have a smooth and proper scheme $X$ over a complete discrete valuation ring $V$ of mixed characteristic $(0, p)$ with perfect residue field $k$ and fraction field $K$. Let $X_{0}$ denote its special fiber. We write $W(k)$ for the Witt vectors of $k$. Recall the statement of the Crystalline conjecture relating the étale cohomology of the geometric generic fiber $X_{\bar{K}}$ as a representation of the Galois group of the field $K$ and the de Rham cohomology of $X_{K}$ with some extra structures.

Conjecture 1.1. There exists a natural p-adic period isomorphism

$$
\alpha_{i}: \quad H^{i}\left(X_{\bar{K}}, \mathbf{Q}_{p}\right) \otimes B_{\mathrm{cr}} \simeq H_{\mathrm{cr}}^{i}\left(X_{0} / W(k)\right) \otimes B_{\mathrm{cr}}
$$

that is $B_{\mathrm{cr}}$-linear, Galois equivariant, compatible with Frobenius, and induces an isomorphism on filtrations after passing to $B_{d R}$.

Here, $B_{\mathrm{cr}}$ is Fontaine's crystalline ring of periods. It is equipped with Galois action and Frobenius. It maps to another ring of period $B_{d R}$ that is equipped with a descending filtration. The filtration on the right hand side comes from that and the Hodge filtration on the crystalline cohomology $H_{\mathrm{cr}}^{i}\left(X_{0} / W(k)\right)$ tensored with $K$ (a group that is known to be isomorphic to the de Rham cohomology $\left.H_{d R}^{i}\left(X_{K} / K\right)\right)$. As a corollary one gets that the étale cohomology groups as Galois representations can be recovered from the crystalline cohomology

$$
\tilde{\alpha}_{i}: H^{i}\left(X_{\bar{K}}, \mathbf{Q}_{p}\right) \simeq\left(H_{\mathrm{cr}}^{i}\left(X_{0} / W(k)\right) \otimes B_{\mathrm{cr}}\right)^{\phi=1} \cap F^{0}\left(H_{d R}^{i}\left(X_{K} / K\right) \otimes B_{d R}\right) .
$$

It is natural to wonder how unique the $p$-adic period isomorphism is. In the above conjecture one often postulates that the period morphism should preserve Chern classes of vector bundles. It turns out that if one postulates instead that the period morphism should be compatible with higher Chern classes from the 
$p$-adic $K$-theory groups $K_{j}\left(X_{\bar{V}}, \mathbf{Q}_{p}\right)$ one gets that there is a unique $p$-adic period morphism having all the required properties. The higher Chern classes considered are the étale ones into $H^{i}\left(X_{\bar{K}}, \mathbf{Q}_{p}\right)$ and the syntomic ones into

$H^{i}\left(X_{\bar{V}}, S(r)_{\mathbf{Q}_{p}}\right) \simeq\left(H_{\mathrm{cr}}^{i}\left(X_{0} / W(k)\right) \otimes B_{\mathrm{cr}}\right)^{\phi=p^{r}} \cap F^{r}\left(H_{d R}^{i}\left(X_{K} / K\right) \otimes B_{d R}\right), \quad i \leq r$.

We prove

Theorem 1.2. There exists a unique natural p-adic period isomorphism

$$
\alpha_{i}: \quad H^{i}\left(X_{\bar{K}}, \mathbf{Q}_{p}\right) \otimes B_{\mathrm{cr}} \simeq H_{\mathrm{cr}}^{i}\left(X_{0} / W(k)\right) \otimes B_{\mathrm{cr}}
$$

that is $B_{\mathrm{cr}}$-linear, Galois equivariant, compatible with Frobenius, induces an isomorphism on filtrations after passing to $B_{d R}$, and is compatible with the étale and syntomic higher Chern classes from p-adic K-theory.

This theorem follows from our construction of the $p$-adic period morphism in [23]. We have shown there that the higher Chern classes induce an isomorphism between the étale cohomology groups $H^{i}\left(X_{\bar{K}}, \mathbf{Q}_{p}\right)$ and certain gamma graded pieces of the $p$-adic $K$-theory groups of the geometric generic fiber $K_{j}\left(X_{\bar{K}}, \mathbf{Q}_{p}\right)$. The last groups, in turn, are isomorphic to the $p$-adic $K$-theory groups of the integral model $K_{j}\left(X_{\bar{V}}, \mathbf{Q}_{p}\right)$. Hence to give the period map $\tilde{\alpha}_{i}$ from the étale cohomology to the syntomic cohomology is the same as to give a map from the $p$-adic $K$-theory of the integral model to the syntomic cohomology. If we impose compatibility of the $p$-adic period map with higher Chern classes this morphism has to be equal to the syntomic higher Chern classes.

Rational crystalline conjecture was proved in the above generality by three different methods. There is the syntomic method due to Fontaine-Messing [11], Kato-Messing [19], and Tsuji [31]. Then there is the almost étale method of Faltings [6]. Finally there is the motivic method of the author [23]. In the syntomic method the period map $\tilde{\alpha}_{i}$ from the syntomic cohomology to the étale cohomology is defined as the cospecialization map on the syntomic-étale site. It is proved to be an isomorphism via a detailed study of the sheaves of $p$-adic nearby cycles that turn out to be isomorphic to certain syntomic sheaves. In the almost étale method the period map $\alpha_{i}$ itself is defined by evaluating acyclic crystalline resolutions on sheaves of crystalline periods $B_{\mathrm{cr}}^{+}$on certain "étale in characteristic 0" site. That gives a map from the crystalline cohomology to the cohomology of the sheaves $B_{\mathrm{cr}}^{+}$on this site. Almost étale theory shows now 
that this cohomology is almost isomorphic to the étale cohomology tensored with the usual ring of periods $B_{\mathrm{cr}}^{+}$. The motivic method we discussed above: to get the $p$-adic period map $\tilde{\alpha}_{i}$, this time from the étale cohomology to the syntomic cohomology, we first use the étale higher Chern classes to pass from the étale cohomology to the $p$-adic $K$-theory of the geometric generic fiber, then we go to the $p$-adic $K$-theory of the integral model, and follow that with the syntomic higher Chern classes. In all these methods, the fact that the induced period map $\alpha_{i}$ is an isomorphism follows from the Poincaré duality and compatibility with the (usual) Chern classes.

Checking compatibility of the above $p$-adic period maps with higher Chern clases yields

Corollary 1.3. The p-adic period morphisms defined using the syntomic, almost étale and motivic constructions are equal.

We obtain analogous results for the integral $p$-adic period morphisms as well as the rational semistable ones.

Acknowledgments. Parts of this paper were written during my visits to University of Cambridge, University of Tokyo, and Kyoto University. I would like to thank these institutions and especially my hosts: Tony Scholl, Takeshi Saito, and Kazuya Kato for their hospitality.

Throughout the paper, let $p$ be a fixed prime, let $\bar{K}$ denote a chosen algebraic closure of a field $K$, and, for a scheme $X$, let $X_{n}=X \otimes \mathbf{Z} / p^{n}$. All the log-schemes are assumed to be fine.

\section{Preliminaries}

2.1. Rings of periods. Let $V$ be a complete discrete valuation ring with fraction field $K$ of characteristic 0 and with perfect residue field $k$ of characteristic $p$. Let $W(k)$ be the ring of Witt vectors of $k$ with fraction field $K_{0}$. Set $G_{K}=$ $\operatorname{Gal}(\bar{K} / K)$, and let $\sigma$ be the absolute Frobenius on $W(\bar{k})$. For a $V$-scheme $X$, let $X_{0}$ denote the special fiber of $X$. We will denote by $V, V^{\times}$, and $V^{0}$ the scheme $\operatorname{Spec}(V)$ with the trivial, canonical (i.e., associated to the closed point), and $(\mathbf{N} \rightarrow V, 1 \mapsto 0)$ log-structure respectively, and, for a log-scheme $X^{\times}$, we will denote by $X$ the underlying scheme. 
Let us recall the definitions of the rings $B_{\mathrm{cr}}$ and $B_{d R}$ of Fontaine [8], [9], [11]. We have

$$
B_{\mathrm{cr}, n}^{+}=H_{\mathrm{cr}}^{0}\left(\operatorname{Spec}\left(\bar{V}_{n} / W_{n}(k)\right)\right), \quad B_{\mathrm{cr}}^{+}=\operatorname{proj} \lim B_{\mathrm{cr}, n}^{+}, \quad B_{\mathrm{cr}}=B_{\mathrm{cr}}^{+}\left[t^{-1}, p^{-1}\right],
$$

where $\bar{V}$ is the integral closure of $V$ in $\bar{K}$ and $t$ is a certain element of $B_{\mathrm{cr}}^{+}$that will be defined below. The ring $B_{\mathrm{cr}}^{+}$is a topological $W(k)$-module equipped with a Frobenius $\phi$ coming from the crystalline cohomology, filtration $F^{n} B_{\mathrm{cr}}^{+}$by the completed devided powers of the canonical PD-ideal of $B_{\text {cr }}^{+}$(or their modifications - see below), and a natural $G_{K}$-action. We have $\phi(t)=p t$ and $G_{K}$ acts on $t$ via the cyclotomic character.

The canonical morphism $\theta: B_{\mathrm{cr}, n}^{+} \rightarrow \bar{V}_{n}$ is surjective. Let $J_{\mathrm{cr}, n}$ denote its kernel. Let

$$
B_{d R}^{+}=\underset{r}{\operatorname{proj}} \lim \left(\mathbf{Q} \otimes \underset{n}{\operatorname{proj}} \lim B_{\mathrm{cr}, n}^{+} / J_{\mathrm{cr}, n}^{[r]}\right), \quad B_{d R}=B_{d R}^{+}\left[t^{-1}\right] .
$$

The ring $B_{d R}^{+}$has a discrete valuation given by powers of $t$. Its quotient field is $B_{d R}$. We will denote by $F^{n} B_{d R}$ the filtration induced on $B_{d R}$ by powers of $t$.

The above generalizes to certain $V$-algebras [6, II], [31, 1]. First, let $R$ be a smooth $V$-algebra such that $R / p R \neq 0$. Consider the $p$-adic completion $\widehat{R}$. For simplicity, we will assume that $\operatorname{Spec}(R / p R)$ is connected, which implies that $\widehat{R}$ is a normal domain. In general, $\widehat{R}$ is a product of normal domains and what follows applies to each factor. Recall that $R$ is called small if there is an étale map $V\left[T_{1}^{ \pm 1}, \ldots, T_{d}^{ \pm 1}\right] \rightarrow R$. If $R$ is small, Frobenius is surjective on $\widehat{\widehat{R}} / p$.

For small $R$, the crystalline rings of periods can be simply defined by setting

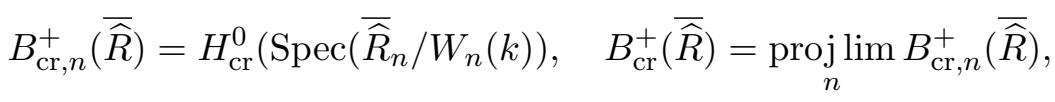

where $\widehat{\widehat{R}}$ is the normalization of $\widehat{R}$ in the maximal étale extension of $\widehat{R}[1 / p]$. In particular $B_{\mathrm{cr}}^{+}(V)=B_{\mathrm{cr}}^{+}$. For general smooth $V$-algebras, we have a more explicit construction. Let $S=\operatorname{proj} \lim \widehat{\widehat{R}} / p$, where the maps in the projective system are the $p$-th power maps. With addition and multiplication defined coordinatewise $S$ is a ring of characteristic $p$. The Frobenius of $S$ is bijective, so that the ring of Witt vectors $W(S)$ is $p$-torsion free, complete and separated for the $p$-adic topology. There is a homomorphism $\theta$ from $W(S)$ to $\overline{\widehat{R}}^{\wedge}: \theta$ maps $\left(x_{0}, x_{1}, \ldots\right) \in W(S)$, $x_{n}=\left(x_{n m}\right) \in S$, to the limit over $m$ of $\hat{x}_{0 m}^{p^{m}}+p \hat{x}_{1 m}^{p^{m-1}}+\cdots+p^{m} \hat{x}_{m m}$, where `means a lift from $\widehat{\widehat{R}} / p$ to $\widehat{\widehat{R}}$. This is a surjection if Frobenius is surjective on 
$\widehat{\widehat{R}} / p$. The kernel of $\theta$ is generated by $\xi=[(p)]+p[(-1)]$, where $(p),(-1) \in S$ are the reductions mod $p$ of sequences of $p$-power roots of $p$ and $(-1)$ respectively (if $p \neq 2$ we may and will choose $(-1)=-1)$.

The ring $B_{\mathrm{cr}}^{+}(\widehat{R})$ is then the $p$-adic completion of the devided power envelope $D_{\xi}(W(S))$ of the ideal $\xi W(S)$ in $W(S)$. Let $J_{\text {cr }}$ denote the PD ideal of $D_{\xi}(W(S))$. $B_{\mathrm{cr}}^{+}(\widehat{\widehat{R}})$ is an algebra over $B_{\mathrm{cr}}^{+}(V)$ having the following properties:

(1) the Frobenius automorphism of $S$ induces an automorphisms $\phi$ of $W(S)$ and $B_{\mathrm{cr}}^{+}(\widehat{\widehat{R}})$;

(2) $B_{\mathrm{cr}}^{+}(\widehat{\widehat{R}})$ is equipped with a decreasing separated filtrations $F^{n} B_{\mathrm{cr}}^{+}(\overline{\widehat{R}})$ and $F_{p}^{n} B_{\mathrm{cr}}^{+}(\widehat{\widehat{R}})$ such that $\phi\left(F_{p}^{n} B_{\mathrm{cr}}^{+}(\widehat{\widehat{R}})\right) \subset p^{n} B_{\mathrm{cr}}^{+}(\widehat{\widehat{R}}): F^{n} B_{\mathrm{cr}}^{+}(\widehat{\widehat{R}})$ is the closure of the $n$-th divided power of $J_{\text {cr }}$ and $F_{p}^{n} B_{\text {cr }}^{+}(\widehat{\widehat{R}})$ is the closure of the ideal consisting of those elements in the $n$-th divided power of $J_{\text {cr }}$ whose $\phi$ image is divisible by $p^{n}$; for $n \leq p-1, F_{p}^{n} B_{\mathrm{cr}}^{+}(\widehat{\widehat{R}})=F^{n} B_{\mathrm{cr}}^{+}(\widehat{\widehat{R}})$;

(3) the Galois group $\operatorname{Gal}(\widehat{\widehat{R}} / \widehat{R})$ acts on $B_{\mathrm{cr}}^{+}(\widehat{\widehat{R}})$; the action is continuous, commutes with $\phi$ and preserves the filtrations;

To define the element $t \in F^{1} B_{\mathrm{cr}}^{+}$, choose a sequence of $p$-power roots of unity in $\bar{V}: \zeta_{0}=1, \zeta_{m+1}=\zeta_{m}, \zeta_{1} \neq 1$. Let us fix such a sequence. Take $\zeta=\left(\zeta_{m}\right)$ and $\pi_{\zeta}=[\zeta]-1 \in W(S)$. Set

$$
t=\log ([\zeta])=\sum_{m \geq 1}(-1)^{m-1}(m-1) ! \pi_{\zeta}^{[m]} .
$$

For small $R$ and $r \geq 0$, we have [22, Prop. 5.1] the following fundamental exact sequence of Galois modules

$$
0 \rightarrow \mathbf{Z}_{p} t^{\{r\}} \rightarrow F_{p}^{r} B_{\mathrm{cr}}^{+}(\widehat{\widehat{R}}) \stackrel{p^{-r} \phi-1}{\longrightarrow} B_{\mathrm{cr}}^{+}(\overline{\widehat{R}}) \rightarrow 0
$$

where $t^{\{r\}}=t^{k(r)}\left(t^{p-1} / p\right)^{[q(r)]}$ for $r=(p-1) q(r)+k(r), 0 \leq k(r)<(p-1)$.

One can carry out the above explicite construction of $B_{\mathrm{cr}}^{+}(A)$ for any noetherian $V$-algebra $A$ such that $A / p A \neq 0, A$ is a normal domain, flat over $V$, containing all $p$-power roots of $-p[31,1.1]$. We also have a cohomological intepretation. Let $\widetilde{A}$ denote the image of the map $\theta: W\left(S_{A}\right) \rightarrow \widehat{A}$, where $S_{A}$ denotes the analog of $S$ for $A$. For every $n>0$, we get a PD-thickening $\operatorname{Spec}\left(\widetilde{A}_{n}\right) \hookrightarrow \operatorname{Spec}\left(B_{\mathrm{cr}, n}^{+}(A)\right)$, hence an object of the crystalline site of $\widetilde{A}_{n}$ over $W_{n}(k)$. One checks that this is 
a final object [31, A1.5]. In particular, the natural map

$$
H_{\mathrm{cr}}^{0}\left(\widetilde{A}_{n} / W_{n}(k), J_{n}^{[r]}\right) \stackrel{\sim}{\rightarrow} F^{r} B_{\mathrm{cr}, n}^{+}(A)
$$

is an isomorphism.

Define the elements $q$ and $q^{\prime}$ of $W\left(S_{A}\right)$ by $q=\sum_{a \in \mathbf{F}_{p}}\left[\zeta^{[a]}\right]$ and $q_{1}=\phi^{-1}(q)$. In the case $\operatorname{Spec}(A / p A)$ is connected and the maps $q_{1}^{n}-\phi: S_{A} \rightarrow S_{A}$ are surjective for all $n \geq 0$, we have the following analog of the fundamental exact sequence $[31,1.2 .4]$

$$
0 \rightarrow \mathbf{Z}_{p} t^{\{r\}} \rightarrow F_{p}^{r} B_{\mathrm{cr}}^{+}(A) \stackrel{p^{-r} \phi-1}{\longrightarrow} B_{\mathrm{cr}}^{+}(A) \rightarrow 0 .
$$

We will need the crystalline interpretation of the ring $B_{\text {st }}^{+}$(see [18], [31]). The definitions of all the period rings below depend on the choice of a uniformizer $\pi$ of $V$. Let us fix such $\pi$. It has minimal equation $f(\pi)=0$, where

$$
f(X)=X^{e}+a_{0} X^{e-1}+\ldots a_{1} X+a_{0}
$$

is an Eisenstein polynomial (hence all $a_{i}$ 's are divisible by $p$ and $a_{0}$ is not divisible by $\left.p^{2}\right)$. Let $R_{V, n}^{\times}$denote the PD-envelope of the ring $W_{n}(k)[X]^{\times}(\log$-structure given by $X)$ with respect to the exact closed immersion $W_{n}(k)[X]^{\times} \rightarrow V_{n}^{\times}$, $X \rightarrow \pi$. We have $V=W_{n}(k)[X] / f(X)$ and $R_{V, n}^{\times}$is obtained by adjoining divided powers $f(X)^{n} / n$ ! or $X^{e n} / n$ !. It has PD-filtration $F^{k}\left(R_{V, n}^{\times}\right)$by the ideals generated by divided powers $f(X)^{n} / n$ ! for $n \geq k$ and the log-structure is associated to $\left(\mathbf{N} \rightarrow R_{V, n}, 1 \rightarrow X\right)$. Frobenius acts by $\mapsto X^{p}$ and ( $W(k)$-linear) monodromy by $N(X)=X$.

Set

$$
\widehat{B}_{\mathrm{st}, n}^{+}=H_{\mathrm{cr}}^{0}\left(\bar{V}_{n}^{\times} / R_{V, n}^{\times}\right), \quad \widehat{B}_{\mathrm{st}}^{+}=\underset{n}{\operatorname{proj} \lim } H_{\mathrm{cr}}^{0}\left(\bar{V}_{n}^{\times} / R_{V, n}^{\times}\right) .
$$

The ring $\widehat{B}_{\mathrm{st}, n}^{+}$has a natural action of $G_{K}$, Frobenius $\phi$, and a monodromy operator $N$. It is also equipped with a PD-filtration $F^{i} \widehat{B}_{\mathrm{st}, n}^{+}=H_{\mathrm{cr}}^{0}\left(\bar{V}_{n}^{\times} / R_{V, n}^{\times}, J_{n}^{[i]}\right)$. We have a morphism $B_{\mathrm{cr}, n}^{+} \rightarrow \widehat{B}_{\mathrm{st}, n}^{+}$induced by the map $H_{\mathrm{cr}}^{0}\left(\bar{V}_{n} / W_{n}(k)\right) \rightarrow$ $H_{\mathrm{cr}}^{0}\left(\bar{V}_{n}^{\times} / R_{V, n}^{\times}\right)$. It is compatible with the Galois action, the Frobenius, and the filtration. The following lemma describes the structure of $\widehat{B}_{\mathrm{st}, n}^{+}$explicitely.

Lemma 2.1. ([18, Proposition 3.3]) Let $s$ be a $p^{n}$ 'th root of $\pi$ contained in $\bar{K}$. 
(1) The PD-morphism $B_{\mathrm{cr}, n}^{+}<Y>\stackrel{\sim}{\rightarrow} \widehat{B}_{\mathrm{st}, n}^{+}$that sends $Y$ to $v_{s}-1$ is an isomorphism. We have $\tilde{s}^{p^{n}} v_{s}=X$ and $v_{\zeta s}=\tilde{\zeta}^{-p^{n}} v_{s}$ for any $p^{n}{ }^{\prime}$ th root $\zeta$ of 1 in $\bar{V}$, where denotes a lifting from $\bar{V}_{n}$ to $B_{\mathrm{cr}, n}^{+}$.

(2) The monodromy operator $N$ is a $B_{\mathrm{cr}, n}^{+}$-linear homomorphism characterized by $N(1)=0$ and $N\left(\left(v_{s}-1\right)^{[i]}\right)=\left(v_{s}-1\right)^{[i-1]} v_{s}$.

(3) The Frobenius $\phi$ is a PD-linear homomorphism such that $\phi\left(v_{s}\right)=v_{s}^{p}$.

(4) The Galois action of $G_{K}$ is characterized by $\sigma\left(v_{s}\right)=v_{\sigma(s)}, \sigma \in G_{K}$.

The natural map $R_{V, n}^{\times} \rightarrow \widehat{B}_{\mathrm{st}, n}^{+}$is compatible with all the structures. We need to explain the definition of the element $v_{s}$ in the above lemma. There is a particularly nice (equivalent) definition of $\widehat{B}_{\mathrm{st}, n}^{+}$in [31, p.256]. It shows that we can view $\widehat{B}_{\mathrm{st}, n}^{+}$as the $\mathrm{PD}$-envelope of the closed immersion

$$
\bar{V}_{n}^{\times} \hookrightarrow B_{\mathrm{cr}, n}^{+, \times} \times_{W_{n}(k)} W_{n}(k)[X]^{\times}
$$

defined by the map $\theta: B_{\mathrm{cr}, n}^{+} \rightarrow \bar{V}_{n}$ and the projection $W_{n}(k)[X] \rightarrow \bar{V}_{n}, X \mapsto \pi$. This makes $\bar{V}_{n}^{\times} \hookrightarrow \widehat{B}_{\mathrm{st}, n}^{+}$into a PD-thickening in the crystalline site of $\bar{V}_{n}^{\times} / R_{V, n}^{\times}$. Here $B_{\mathrm{cr}, n}^{+, \times}$is a PD-thickening in the crystalline site of $\bar{V}_{n}^{\times} / R_{V, n}^{\times}$given by $B_{\mathrm{cr}, n}^{+}$ and the map $\theta$ equipped with the following $\log$-structure. Fix a sequence of $p$ power roots of $\pi$ in $\bar{V}: s_{0}=\pi, s_{n+1}^{p}=s_{n}$. Denote by $[\pi]$ the associated element of $B_{\mathrm{cr}}^{+}$. The log-structure of $B_{\mathrm{cr}, n}^{+, \times}$is given by $\left(\mathbf{N} \rightarrow B_{\mathrm{cr}}^{+}, 1 \mapsto[\pi]\right)$. We easily see that there is a unique element $v_{[\pi]} \in 1+F^{1} \widehat{B}_{\mathrm{st}, n}^{+}$such that $v_{[\pi]}[\pi]=X$. That gives our $v_{s}$ in the above lemma.

The element $[\pi] \pi^{-1}$ is contained in $1+F^{1} B_{d R}$ and hence $\log \left([\pi] \pi^{-1}\right)$ converges in $B_{d R}^{+}$to an element $u_{[\pi]}$. Let $B_{\mathrm{st}}^{+}$denote the subring of $B_{d R}$ generated by $B_{\mathrm{cr}}^{+}[1 / p]$ and $u_{[\pi]}$. Fontaine shows that $u_{[\pi]}$ is transcendental over $B_{\mathrm{cr}}^{+}[1 / p]$. Hence $B_{\mathrm{st}}^{+}$is a polynomial algebra in one variable over $B_{\mathrm{cr}}^{+}[1 / p]$. The action of $G_{K}$ on $B_{d R}^{+}$ restricts well to $B_{\mathrm{st}}^{+}$: we have $\sigma\left(u_{[\pi]}\right)=u_{[\pi]}+\log (\beta(\sigma)), \sigma \in G_{K}$, where $\beta(\sigma)$ is defined by $\sigma([\pi])=\beta(\sigma)[\pi]$. The Frobenius $\phi$ extends to $B_{\text {st }}^{+}$by $\phi\left(u_{[\pi]}\right)=p u_{[\pi]}$ and one defines the monodromy operator $N: B_{\mathrm{st}}^{+} \rightarrow B_{\mathrm{st}}^{+}$as the unique $B_{\mathrm{cr}}^{+}[1 / p]$ derivation such that $N u_{[\pi]}=-1$. We have $N \phi=p \phi N$. Let $B_{\mathrm{st}}=B_{\mathrm{cr}}\left[u_{[\pi]}\right]$. Different choices of the uniformizer $\pi$ yield isomorphic rings $B_{\mathrm{st}}^{+}$, so we can and we will identify them via these isomorphisms. The dependence on $\pi$ will then be encoded in the morphism $\iota_{\pi}: B_{\mathrm{st}}^{+} \hookrightarrow B_{d R}^{+}$. 
Kato $[18,3.7]$ shows that the $\operatorname{ring} B_{\mathrm{st}}^{+}$is canonically (and compatibly with all the structures) isomorphic to the elements of $\widehat{B}_{\mathrm{st}}^{+}[1 / p]$ annihilated by a power of the monodromy operator $N$.

Lemma 2.2. There is a canonical $B_{\mathrm{cr}}^{+}[1 / p]$-isomorphism

$$
B_{\mathrm{st}}^{+} \stackrel{\sim}{\rightarrow} \widehat{B}_{\mathrm{st}}^{+}[1 / p]^{N-n i l p}, \quad u_{s} \mapsto 1 \otimes\left\{\log \left(v_{s_{n}}^{-1}\right)\right\}_{n \geq 1},
$$

compatible with the action of $G_{K}, \phi$ and $N$.

We have projections

$$
p_{1}: \widehat{B}_{\mathrm{st}, n}^{+} \rightarrow B_{\mathrm{cr}, n}^{+}, \quad v_{s_{n}} \mapsto 1 ; \quad p_{0}: B_{\mathrm{st}}^{+} \rightarrow B_{\mathrm{cr}}^{+}[1 / p], \quad u_{s} \mapsto 0 .
$$

Since $\widehat{B}_{\mathrm{st}, n}^{+}$is the final object in the crystalline site of $\bar{V}_{n}^{\times}$over $R_{V, n}^{\times}, p_{1}$ is the unique map of $\widehat{B}_{\mathrm{st}, n}^{+}$to $B_{\mathrm{cr}, n}^{+}$as PD-thickenings of $\bar{V}_{n}^{\times}$over $R_{V, n}^{\times}$. In particular it is compatible with filtrations. The projections are compatible with the map $B_{\mathrm{st}}^{+} \rightarrow \widehat{B}_{\mathrm{st}}^{+}[1 / p]$ and induce the identity on $B_{\mathrm{cr}, n}^{+}$and $B_{\mathrm{cr}}^{+}[1 / p]$. Notice though that they are clearly not (!) Galois equivariant. However their restrictions to $\left(\widehat{B}_{\mathrm{st}, n}^{+}\right)^{N=0}=B_{\mathrm{cr}, n}^{+}$and $\left(B_{\mathrm{st}}^{+}\right)^{N=0}=B_{\mathrm{cr}}^{+}[1 / p]$ are. We will see a more general version of this phenomena in log-comparison theorems below.

2.2. Syntomic cohomology. Let $X$ be a flat finite type scheme over $W(k)$. Recall the differential definition [16] of syntomic cohomology of Fontaine-Messing [11]. Assume first that we have an immersion $i: X \hookrightarrow Z$ over $W(k)$ such that $Z$ is a smooth $W(k)$-scheme endowed with a compatible system of liftings of the Frobenius $\left\{F_{n}: Z_{n} \rightarrow Z_{n}\right\}$. Let $D_{n}=D_{X_{n}}\left(Z_{n}\right)$ be the PD-envelope of $X_{n}$ in $Z_{n}$ (compatible with the canonical PD-structure on $\left.p W_{n}(k)\right)$ and $J_{D_{n}}$ the ideal of $X_{n}$ in $D_{n}$. Set $J_{D_{n}}^{<r>}:=\left\{a \in J_{D_{n+s}}^{[r]} \mid \phi(a) \in p^{r} \mathcal{O}_{D_{n+s}}\right\} / p^{n}$ for some $s \geq r$. For $0 \leq r \leq p-1, J_{D_{n}}^{<r>}=J_{D_{n}}^{[r]}$. This definition is independent of $s$. Consider the following complexes

$$
\begin{aligned}
& S_{n}(r)_{X}:=\operatorname{Cone}\left(J_{D_{n}}^{<r-\cdot>} \otimes \Omega_{Z_{n} / W_{n}(k)} \stackrel{1-\phi_{r}}{\longrightarrow} \mathcal{O}_{D_{n}} \otimes \Omega_{Z_{n} / W_{n}(k)}\right)[-1], \\
& S_{n}^{\prime}(r)_{X}:=\operatorname{Cone}\left(J_{D_{n}}^{[r-\cdot]} \otimes \Omega_{Z_{n} / W_{n}(k)} \stackrel{p^{r}-\phi}{\longrightarrow} \mathcal{O}_{D_{n}} \otimes \Omega_{Z_{n} / W_{n}(k)}\right)[-1],
\end{aligned}
$$

where $\phi_{r}$ is " $\phi / p^{r} "$, (see $[31,2.1]$ for details). The complexes $S_{n}(r)_{X}, S_{n}^{\prime}(r)_{X}$ are, up to canonical quasi-isomorphisms, independent of the choice of $i$ and $\left\{F_{n}\right\}$. There is a natural map $S_{n}^{\prime}(r)_{X} \rightarrow S_{n}(r)_{X}$, whose kernel and cokernel is annihilated by $p^{r}$. The reader will find more canonical definition of these complexes in the next chapter. 
In general, immersions as above exist étale locally, and we define $S_{n}(r)_{X} \in$

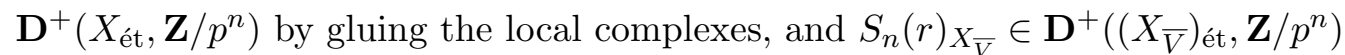
as the inductive limit of $S_{n}(r)_{X_{V^{\prime}}}$, where $V^{\prime}$ varies over the integral closures of $V$ in all finite extensions of $K$ in $\bar{K}$. All these complexes are equipped with a well-behaved product. Set

$$
H^{i}\left(X, S_{n}(r)\right):=H_{\text {ét }}^{i}\left(X, S_{n}(r)_{X}\right), \quad H^{i}\left(X_{\bar{V}}, S_{n}(r)\right):=H_{\text {ét }}^{i}\left(X_{\bar{V}}, S_{n}(r)_{X_{\bar{V}}}\right) .
$$

Similarly, we define $S_{n}^{\prime}(r)_{X}$ and $S_{n}^{\prime}(r)_{X_{\bar{V}}}$, and the groups $H^{i}\left(X, S_{n}^{\prime}(r)\right), H^{i}\left(X_{\bar{V}}, S_{n}^{\prime}(r)\right)$. For $r \geq 0$, we get the long exact sequences

$$
\begin{aligned}
\ldots \rightarrow H^{i}\left(X_{\bar{V}}, S_{n}(r)\right) \rightarrow H_{\mathrm{cr}}^{i}\left(X_{\bar{V}, n} / W_{n}(k), J_{X_{\bar{V}, n} / W_{n}(k)}^{<r>}\right) \stackrel{1-\phi_{r}}{\longrightarrow} \\
H_{\mathrm{cr}}^{i}\left(X_{\bar{V}, n} / W_{n}(k), \mathcal{O}_{X_{\bar{V}, n} / W_{n}(k)}\right) \rightarrow \ldots \\
\ldots \rightarrow H^{i}\left(X_{\bar{V}}, S_{n}^{\prime}(r)\right) \rightarrow H_{\mathrm{cr}}^{i}\left(X_{\bar{V}, n} / W_{n}(k), J_{X_{\bar{V}, n} / W_{n}(k)}^{[r]} \stackrel{p^{r}-\phi}{\longrightarrow}\right. \\
H_{\mathrm{cr}}^{i}\left(X_{\bar{V}, n} / W_{n}(k), \mathcal{O}_{X_{\bar{V}, n} / W_{n}(k)}\right) \rightarrow \ldots
\end{aligned}
$$

Let now $X^{\times}$be a flat and separated, finite type log-scheme over $W(k)$. Recall [17] that there exists log-crystalline cohomology, which is defined by mimicking the definition of the classical crystalline cohomology. Using it, the above construction of syntomic complexes goes through almost verbatim (see [31, 2.1] for details) to yield the logarithmic analog $S_{n}(r)_{X \times}$ on $X_{\text {ét }}$ and the corresponding log-syntomic cohomology groups

$$
H^{i}\left(X^{\times}, S_{n}(r)\right):=H_{\text {ét }}^{i}\left(X, S_{n}(r)_{X^{\times}}\right), \quad H^{i}\left(X_{\bar{V}}^{\times}, S_{n}(r)\right):=H_{\text {ét }}^{i}\left(X_{\bar{V}}, S_{n}(r)_{X_{\bar{V}}^{\times}}\right) .
$$

Similarly for complexes $S_{n}^{\prime}(r)_{X^{\times}}$. There are natural maps

$$
\varepsilon: H^{i}\left(X, S_{n}(r)\right) \rightarrow H^{i}\left(X^{\times}, S_{n}(r)\right), \quad \varepsilon: H^{i}\left(X, S_{n}(r)\right) \rightarrow H^{i}\left(X^{\times}, S_{n}(r)\right) .
$$

Again, for $r \geq 0$, we get the long exact sequences

$$
\begin{gathered}
\ldots \rightarrow H^{i}\left(X_{\bar{V}}^{\times}, S_{n}(r)\right) \rightarrow H_{\mathrm{cr}}^{i}\left(X_{\bar{V}, n}^{\times} / W_{n}(k), J_{X_{\bar{V}, n} / W_{n}(k)}^{<r>}\right) \stackrel{1-\phi_{r}}{\longrightarrow} \\
H_{\mathrm{cr}}^{i}\left(X_{\bar{V}, n}^{\times} / W_{n}(k), \mathcal{O}_{X_{\bar{V}, n}^{\times} / W_{n}(k)}\right) \rightarrow \ldots, \\
\ldots \rightarrow H^{i}\left(X_{\bar{V}}^{\times}, S_{n}^{\prime}(r)\right) \rightarrow H_{\mathrm{cr}}^{i}\left(X_{\bar{V}, n}^{\times} / W_{n}(k), J_{X_{\bar{V}, n} / W_{n}(k)}^{[r]}\right) \stackrel{p^{r}-\phi}{\longrightarrow} \\
H_{\mathrm{cr}}^{i}\left(X_{\bar{V}, n}^{\times} / W_{n}(k), \mathcal{O}_{X_{\bar{V}, n} / W_{n}(k)}\right) \rightarrow \ldots
\end{gathered}
$$


Lemma 2.3. For $X$ smooth and proper over $W(k)$ and $p-2 \geq r \geq i$, the natural map

$$
H^{i}\left(X_{\bar{V}}, S_{n}(r)\right) \stackrel{\sim}{\rightarrow} F^{r} H_{\mathrm{cr}}^{i}\left(X_{\bar{V}, n} / W_{n}(k), \mathcal{O}_{X_{\bar{V}, n} / W_{n}(k)}\right)^{\phi_{r}=1}
$$

is an isomorphism.

Proof. By [11, III.1.3] and [11, III.1,II.2], respectively, we have the following isomorphisms

$$
\begin{aligned}
H_{\mathrm{cr}}^{i}\left(X_{\bar{V}, n} / W_{n}(k)\right) & \simeq H_{\mathrm{cr}}^{i}\left(X / W(k), \mathcal{O}_{X_{n} / W_{n}(k)}\right) \otimes B_{\mathrm{cr}, n}^{+}, \\
H_{\mathrm{cr}}^{i}\left(X_{\bar{V}, n} / W_{n}(k), J_{X_{\bar{V}, n}^{[r]} / W_{n}(k)}\right) & \simeq F^{r}\left(H_{\mathrm{cr}}^{i}\left(X_{n} / W_{n}(k)\right) \otimes B_{\mathrm{cr}, n}^{+}\right)
\end{aligned}
$$

By [11, II.2.7], the filtered Frobenius module $H_{\mathrm{cr}}^{i}\left(X_{n} / W_{n}(k)\right)$ belongs to the Fontaine-Laffaille category $\mathcal{F} \mathcal{M}(r)$ [10]. This implies that the map

$$
F^{r}\left(H_{\mathrm{cr}}^{i}\left(X_{n} / W_{n}(k)\right) \otimes B_{\mathrm{cr}, n}^{+}\right) \stackrel{1-\phi_{r}}{\longrightarrow} H_{\mathrm{cr}}^{i}\left(X_{n} / W_{n}(k)\right) \otimes B_{\mathrm{cr}, n}^{+}
$$

is surjective and our isomorphism follows from the exact sequence (2.3).

Rationally, we have the following computation.

Lemma 2.4. Let $r \geq i$. For $X$ smooth and proper over $V$, we have a canonical Galois equivariant isomorphism

$\mathbf{Q} \otimes$ proj $\lim H^{i}\left(X_{\bar{V}}, S_{n}^{\prime}(r)\right) \stackrel{\sim}{\rightarrow}\left(H_{\mathrm{cr}}^{i}\left(X_{0} / W(k)\right) \otimes B_{\mathrm{cr}}\right)^{\phi=p^{r}} \cap F^{r}\left(H_{d R}^{i}\left(X_{K} / K\right) \otimes_{K} B_{d R}\right)$.

Proof. Here we consider $H_{\mathrm{cr}}^{i}\left(X_{0} / W(k)\right) \otimes B_{\mathrm{cr}}^{+}[1 / p]$ a subspace of $H_{d R}^{i}\left(X_{K} / K\right) \otimes_{K}$ $B_{d R}^{+}$via the isomorphism $H_{\mathrm{cr}}^{i}\left(X_{0} / W(k)\right) \otimes_{W(k)} K \simeq H_{d R}^{i}\left(X_{K} / K\right)$ and the injection $B_{\text {cr }} \otimes_{W(k)} K \hookrightarrow B_{d R}$. Recall that Kato and Messing have constructed the following isomorphisms [19, 1.2,1.3]

$$
\begin{aligned}
& H_{\mathrm{cr}}^{i}\left(X_{0} / W(k)\right) \otimes B_{\mathrm{cr}}^{+}[1 / p] \stackrel{\sim}{\rightarrow} \mathbf{Q} \otimes H_{\mathrm{cr}}^{i}\left(X_{\bar{V}} / W(k)\right), \\
& \left(H_{d R}^{i}\left(X_{K} / K\right) \otimes B_{d R}^{+}\right) / F^{r}\left(H_{d R}^{i}\left(X_{K} / K\right) \otimes B_{d R}^{+}\right) \simeq \\
& \mathbf{Q} \otimes \underset{n}{\operatorname{proj}} \lim H_{\mathrm{cr}}^{i}\left(X_{\bar{V}, n} / W_{n}(k), \mathcal{O}_{X_{\bar{V}, n} / W_{n}(k)} / J_{X_{\bar{V}, n} / W_{n}(k)}^{[r]}\right) .
\end{aligned}
$$

It follows that the image of the map

$$
\begin{aligned}
\mathbf{Q} \otimes \underset{n}{\operatorname{proj}} \lim H_{\mathrm{cr}}^{i}\left(X_{\bar{V}, n} / W_{n}(k), \mathcal{O}_{X_{\bar{V}, n} / W_{n}(k)} / J_{X_{\bar{V}, n} / W_{n}(k)}^{[r]}\right) \rightarrow & \\
& \mathbf{Q} \otimes \underset{n}{\operatorname{proj}} \lim H_{\mathrm{cr}}^{i}\left(X_{\bar{V}, n} / W_{n}(k)\right)
\end{aligned}
$$


is equal to $\left(H_{\mathrm{cr}}^{i}\left(X_{0} / W(k)\right) \otimes B_{\mathrm{cr}}^{+}[1 / p]\right)^{\phi=p^{r}} \cap F^{r}\left(H_{d R}^{i}\left(X_{K} / K\right) \otimes_{K} B_{d R}^{+}\right)$. Hence we get a natural map

$\mathbf{Q} \otimes \underset{n}{\operatorname{proj}} \lim H^{i}\left(X_{\bar{V}}, S_{n}^{\prime}(r)\right) \rightarrow\left(H_{\mathrm{cr}}^{i}\left(X_{0} / W(k)\right) \otimes B_{\mathrm{cr}}^{+}[1 / p]\right)^{\phi=p^{r}} \cap F^{r}\left(B_{d R}^{+} \otimes_{K} H_{d R}^{i}\left(X_{K} / K\right)\right)$.

For $r \geq i$, let $\alpha_{i, r}^{T}: H^{i}\left(X_{\bar{K}}, \mathbf{Q}_{p}(r)\right) \rightarrow \mathbf{Q} \otimes \operatorname{proj} \lim _{n} H^{i}\left(X_{\bar{V}}, S_{n}^{\prime}(r)\right)$ be the $p$-adic period morphism defined by Tsuji in [31, 3.1.12, 3.3.4]. It is an isomorphism. We know that the composition of the above two maps

$$
H^{i}\left(X_{K}, \mathbf{Q}_{p}(r)\right) \rightarrow\left(H_{\mathrm{cr}}^{i}\left(X_{0} / W(k)\right) \otimes B_{\mathrm{cr}}\right)^{\phi=p^{r}} \cap F^{r}\left(B_{d R} \otimes_{K} H_{d R}^{i}\left(X_{K} / K\right)\right)
$$

is an isomorphism as well [31, 4.8.1, 4.10.2]. Hence our lemma.

Before stating the analog of the above lemma for log-schemes we need to recall properties of certain cohomology groups and morphisms that appear in $p$-adic comparison theorems for log-schemes. Assume that $X^{\times}$is a fine and saturated $\log$-scheme, $\log$-smooth and proper over $V^{\times}$. We have the log-crystalline cohomology $H_{\mathrm{cr}}^{i}\left(X_{0}^{\times} / W_{n}(k)^{0}\right)$ that is equipped with a (nilpotent) monodromy operator $N$ and a Frobenius $\phi$. We have $N \phi=p \phi N$. If $X_{0}^{\times}$is of Cartier type, the Frobenius is bijective and we have the Hyodo-Kato $K$-isomorphism $[15,5.1]$

$$
\rho: K \otimes_{W(k)} H_{\mathrm{cr}}^{i}\left(X_{0}^{\times} / W(k)^{0}\right) \stackrel{\sim}{\rightarrow} H_{d R}^{i}\left(X_{K}^{\times} / K\right) .
$$

Similarly, we have the groups $H_{\mathrm{cr}}^{i}\left(X_{n}^{\times} / R_{V, n}^{\times}\right)$and a monodromy operator acting on them: there exists a quasi-nilpotent integrable connection ([31, Lemma 4.3.2, Lemma 4.3.6])

$$
\nabla: H_{\mathrm{cr}}^{i}\left(X_{n}^{\times} / R_{V, n}^{\times}\right) \rightarrow H_{\mathrm{cr}}^{i}\left(X_{n}^{\times} / R_{V, n}^{\times}\right) \otimes_{W_{n}(k)} \Omega_{W_{n}(k)[X]^{\times} / W_{n}(k)}^{1}
$$

and we define $\nabla(a)=N(a) \operatorname{dlog} X$. This connection is compatible with Frobenius thus $N \phi=p \phi N$. In the case $X / V$ is proper it also satisfies Griffiths transversality. To see that recall $[7,5]$ that $R \Gamma_{\mathrm{cr}}\left(X_{n}^{\times} / R_{V, n}^{\times}\right)$can be represented by a finite complex of filtered free $R_{V, n}$-modules, where "filtered free"' means isomorphic to a direct sum of copies of $R_{V, n}$ with (possibly) shifted filtrations. The classical argument applies and gives Griffiths transversality on this complex. Hence on cohomology groups as well. It follows that $N\left(F^{k} H_{\mathrm{cr}}^{i}\left(X_{n}^{\times} / R_{V, n}^{\times}\right)\right) \subset$ $F_{\mathrm{cr}}^{k-1} H^{i}\left(X_{n}^{\times} / R_{V, n}^{\times}\right)$. Moreover, all the structures on $H_{\mathrm{cr}}^{i}\left(X_{n}^{\times} / R_{V, n}^{\times}\right)$are compatible with those on $R_{V, n}^{\times}$. There is a natural projection $(X \mapsto 0)$

$$
p_{0}: H_{\mathrm{cr}}^{i}\left(X_{n}^{\times} / R_{V, n}^{\times}\right) \rightarrow H_{\mathrm{cr}}^{i}\left(X_{0}^{\times} / W_{n}(k)^{0}\right)
$$


that commutes with the monodromy. If $X_{0}^{\times}$is of Cartier type, the map $\mathbf{Q} \otimes p_{0}$ has a canonical section [15, Lemma 5.2]

$$
s: H_{\mathrm{cr}}^{i}\left(X_{0}^{\times} / W_{n}(k)^{0}\right)[1 / p] \rightarrow H_{\mathrm{cr}}^{i}\left(X^{\times} / R_{V}^{\times}\right)[1 / p]
$$

compatible with Frobenius and the monodromy operators. It induces an $R_{V^{-}}$ linear isomorphism

$$
R_{V} \otimes_{W(k))} H_{\mathrm{cr}}^{i}\left(X_{0}^{\times} / W_{n}(k)^{0}\right)[1 / p] \stackrel{\sim}{\rightarrow} H_{\mathrm{cr}}^{i}\left(X^{\times} / R_{V}^{\times}\right)[1 / p]
$$

Kato shows $[18,4]$ that there is a long exact sequence

$$
\ldots \rightarrow H_{\mathrm{cr}}^{i}\left(X^{\times} / W_{n}(k)\right) \rightarrow H_{\mathrm{cr}}^{i}\left(X_{n}^{\times} / R_{V, n}^{\times}\right) \stackrel{N}{\rightarrow} H_{\mathrm{cr}}^{i}\left(X_{n}^{\times} / R_{V, n}^{\times}\right) \rightarrow \ldots
$$

It follows that the canonical Galois equivariant map

$$
H_{\mathrm{cr}}^{i}\left(X_{\bar{V}, n}^{\times} / W_{n}(k)\right) \rightarrow\left(H_{\mathrm{cr}}^{i}\left(X_{\bar{V}, n}^{\times} / R_{V, n}^{\times}\right)^{N=0}\right.
$$

induces an isomorphism

$$
H_{\mathrm{cr}}^{i}\left(X_{\bar{V}}^{\times} / W(k)\right)[1 / p] \stackrel{\sim}{\rightarrow}\left(H_{\mathrm{cr}}^{i}\left(X_{\bar{V}}^{\times} / R_{V}^{\times}\right)[1 / p]\right)^{N=0}
$$

Recall that $\widehat{B}_{\mathrm{st}, n}^{+} \simeq H_{\mathrm{cr}}^{0}\left(\bar{V}_{n}^{\times} / R_{V, n}^{\times}\right)$. We also have $H_{\mathrm{cr}}^{i}\left(\bar{V}_{n}^{\times} / R_{V, n}^{\times}\right)$for $i>0[18$, Prop. 3.1]. It follows [18, Lemma 4.2] that cup product induces an isomorphism

$$
\widehat{B}_{\mathrm{st}, n}^{+} \otimes_{R_{V, n}} H_{\mathrm{cr}}^{i}\left(X_{n}^{\times} / R_{V, n}^{\times}\right) \stackrel{\sim}{\rightarrow} H_{\mathrm{cr}}^{i}\left(X_{\bar{V}, n}^{\times} / R_{V, n}^{\times}\right)
$$

compatible with all the structures. For $X_{0}^{\times}$of Cartier type, the above yields an isomorphism

$$
\widehat{B}_{\mathrm{st}}^{+}[1 / p] \otimes H_{\mathrm{cr}}^{i}\left(X_{0}^{\times} / W(k)^{0}\right) \stackrel{\sim}{\rightarrow} H_{\mathrm{cr}}^{i}\left(X_{\bar{V}}^{\times} / R_{V}^{\times}\right)[1 / p]
$$

and, by taking $N$-nilpotent elements, an isomorphism

$$
B_{\mathrm{st}}^{+} \otimes H_{\mathrm{cr}}^{i}\left(X_{0}^{\times} / W(k)^{0}\right) \stackrel{\sim}{\rightarrow}\left(H_{\mathrm{cr}}^{i}\left(X_{\bar{V}}^{\times} / R_{V}^{\times}\right)[1 / p]\right)^{N-n i l p}
$$

Lemma 2.5. Let $r \geq i$. For $X^{\times}$a fine and saturated, log-smooth, vertical, and proper scheme over $V^{\times}$with Cartier type reduction, we have a canonical Galois equivariant isomorphism

$\mathbf{Q} \otimes \underset{n}{\operatorname{proj}} \lim H^{i}\left(X_{\bar{V}}^{\times}, S_{n}^{\prime}(r)\right) \stackrel{\sim}{\rightarrow}\left(H_{\mathrm{cr}}^{i}\left(X_{0}^{\times} / W(k)^{0}\right) \otimes B_{\mathrm{st}}\right)^{N=0, \phi=p^{r}} \cap F^{r}\left(H_{d R}^{i}\left(X_{K} / K\right) \otimes_{K} B_{d R}\right)$. 
Proof. Here we consider $H_{\mathrm{cr}}^{i}\left(X_{0}^{\times} / W(k)^{0}\right) \otimes B_{\mathrm{st}}^{+}$a subspace of $H_{d R}^{i}\left(X_{K} / K\right) \otimes_{K} B_{d R}^{+}$ via the injection $\iota_{\pi}: B_{\mathrm{st}}^{+} \hookrightarrow B_{d R}^{+}$and the Hyodo-Kato isomorphism $\rho$. For $X^{\times}$as above, we have a canonical morphisms

$$
\mathbf{Q} \otimes H_{\mathrm{cr}}^{i}\left(X_{\bar{V}}^{\times} / W(k)\right) \rightarrow\left(B_{\mathrm{st}}^{+} \otimes_{W(k)} H_{\mathrm{cr}}^{i}\left(X_{0}^{\times} / W(k)^{0}\right)\right)^{N=0},
$$

that is compatible with Galois action and Frobenius. Composing with the HyodoKato isomorphism $\rho$ we get a map

$$
\mathbf{Q} \otimes H_{\mathrm{cr}}^{i}\left(X_{\bar{V}}^{\times} / W(k)\right) \rightarrow B_{\mathrm{st}}^{+} \otimes_{K_{0}} H_{d R}^{i}\left(X_{K}^{\times} / K\right),
$$

that is compatible with Galois action, Frobenius and the monodromy operator.

Recall that we have the following crystalline interpretation of $B_{d R}^{+} \otimes_{K} H_{d R}^{i}\left(X_{K} / K\right)$ from [18] (see also [31, 4.7.4]):

$\left(B_{d R}^{+} \otimes_{K} H_{d R}^{i}\left(X_{K} / K\right)\right) / F^{r}\left(B_{d R}^{+} \otimes_{K} H_{d R}^{i}\left(X_{K} / K\right)\right) \stackrel{\sim}{\rightarrow} \mathbf{Q} \otimes H_{\mathrm{cr}}^{i}\left(X_{\bar{V}}^{\times} / V^{\times}, \mathcal{O} / J^{[r]}\right)$.

It follows that there is a natural map

$\mathbf{Q} \otimes \underset{n}{\operatorname{proj}} \lim H^{i}\left(X_{\bar{V}}^{\times}, S_{n}^{\prime}(r)\right) \rightarrow\left(H_{\mathrm{cr}}^{i}\left(X_{0}^{\times} / W(k)^{0}\right) \otimes B_{\mathrm{st}}^{+}\right)^{N=0, \phi=p^{r}} \cap F^{r}\left(B_{d R}^{+} \otimes_{K} H_{d R}^{i}\left(X_{K} / K\right)\right)$.

For $r \geq i$, let $\alpha_{i, r}^{T}: H^{i}\left(X_{\bar{K}}, \mathbf{Q}_{p}(r)\right) \rightarrow \mathbf{Q} \otimes \operatorname{proj} \lim _{n} H^{i}\left(X_{\bar{V}}^{\times}, S_{n}^{\prime}(r)\right)$ be the $p$-adic period morphism defined in [31, 3.1.12, 3.3.4] and in Lemma 4.5 below. It is an isomorphism. We know that the composition of the above two maps

$H^{i}\left(X_{\bar{K}}, \mathbf{Q}_{p}(r)\right) \rightarrow\left(H_{\mathrm{cr}}^{i}\left(X_{0}^{\times} / W(k)^{0}\right) \otimes B_{\mathrm{st}}\right)^{N=0, \phi=p^{r}} \cap F^{r}\left(B_{d R} \otimes_{K} H_{d R}^{i}\left(X_{K} / K\right)\right)$ is an isomorphism as well ([31, 4.8.1, 4.10.2] and Corollary 4.6 below). Hence our lemma.

2.3. Chern classes. For a scheme $X$, let $K_{*}(X), K_{*}^{\prime}(X)$ denote Quillen's higher $K$-theory and $K^{\prime}$-theory groups of $X$, respectively. The corresponding groups with coefficients $\mathbf{Z} / n$, will be denoted by $K_{i}(X, \mathbf{Z} / n)$ and $K_{i}^{\prime}(X, \mathbf{Z} / n)$. For $n$ such that $v_{2}(n)=0$ or $v_{2}(n)>3$, and $v_{2}(n)=0$ or $v_{3}(n)>1$ they have a well-behaved product. For a noetherian regular connected scheme $X$, we have the following $\gamma$-filtrations compatible with products:

$$
\begin{aligned}
& F_{\gamma}^{k} K_{0}(X)= \begin{cases}K_{0}(X) & \text { if } k \leq 0, \\
\left\langle\gamma_{i_{1}}\left(x_{1}\right) \cdots \gamma_{i_{n}}\left(x_{n}\right) \mid \varepsilon\left(x_{1}\right)=\ldots=\varepsilon\left(x_{n}\right)=0, i_{1}+\cdots+i_{n} \geq k\right\rangle & \text { if } k>0,\end{cases} \\
& F_{\gamma}^{k} K_{q}(X)=\left\langle\gamma_{i_{1}}\left(x_{1}\right) \cup \cdots \cup \gamma_{i_{n}}\left(x_{n}\right) \mid x_{i} \in K_{q_{i}}(X), q_{i}>0, i_{1}+\cdots+i_{n} \geq k\right\rangle, \\
& F_{\gamma}^{k} K_{q}\left(X, \mathbf{Z} / p^{n}\right)=\left\langle\gamma_{i_{1}}\left(x_{1}\right) \cup \cdots \cup \gamma_{i_{n}}\left(x_{n}\right) \mid x_{i} \in K_{q_{i}}\left(X, \mathbf{Z} / p^{n}\right), q_{i} \geq 2, i_{1}+\cdots+i_{n} \geq k\right\rangle \text {, } \\
& \text { where } \varepsilon \text { is the augmentation on } K_{0}(X) \text { and } p^{n}>2 \text {. }
\end{aligned}
$$


For $i \geq 0$, there are functorial and compatible families of syntomic Chern classes

$$
\begin{aligned}
& c_{i, j}^{\text {syn }}: K_{j}(X) \rightarrow H^{2 i-j}\left(X, S_{n}(i)\right) \quad \text { for } j \geq 0, \\
& c_{i, j}^{\text {syn }}: K_{j}\left(X, \mathbf{Z} / p^{n}\right) \rightarrow H^{2 i-j}\left(X, S_{n}(i)\right) \quad \text { for } j \geq 2,
\end{aligned}
$$

that are also compatible with the crystalline Chern classes in $H_{\mathrm{cr}}^{2 i-j}\left(X_{n} / W_{n}(k)\right.$, $\left.\mathcal{O}_{X_{n} / W_{n}(k)}\right)$ via the canonical map $H^{2 i-j}\left(X_{n}, S_{n}(i)\right) \rightarrow H_{\mathrm{cr}}^{2 i-j}\left(X_{n} / W_{n}(k), J_{X_{n} / W_{n}(k)}^{<i>}\right)$. Similarly, we have syntomic Chern classes in $H^{2 i-j}\left(X, S_{n}^{\prime}(i)\right)$. Recall the construction of the classes $c_{i, j}^{\text {syn }}$. First, one constructs universal classes $C_{i, l}^{\text {syn }} \in$ $H^{2 i}\left(B . G L_{l} / W(k), S_{n}(i)\right), C_{i, l}^{\text {syn }} \in H^{2 i}\left(B . G L_{l} / W(k), S_{n}^{\prime}(i)\right)$. Recall [14] that

$$
H_{\mathrm{cr}}^{*}\left(B . G L_{l} / W_{n}(k)\right) \simeq H_{d R}^{*}\left(B . G L_{l} / W_{n}(k)\right) \simeq W_{n}(k)\left[x_{1}, \ldots, x_{l}\right],
$$

where the classes $x_{i} \in H_{d R}^{2 i}\left(B . G L_{l} / W_{n}(k)\right)$ are the de Rham Chern classes of the universal locally free sheaf on $B . G L_{l} / W_{n}(k)$ (defined via a projective space theorem). Similarly, for the Hodge cohomology we have

$$
H_{H d g}^{*}\left(B . G L_{l} / W_{n}(k)\right) \simeq W_{n}(k)\left[x_{1}, \ldots, x_{l}\right] .
$$

It follows that the Hodge-de Rham spectral sequence degenerates. Hence

$$
\begin{aligned}
H_{\mathrm{cr}}^{i}\left(B . G L_{l} / W_{n}(k), J_{B . G L_{l} / W_{n}(k)}^{[j]}\right) & \simeq H_{d R}^{i}\left(B . G L_{l} / W_{n}(k), \Omega_{B \cdot G L_{l} / W_{n}(k)}^{\geq j}\right) \\
& =F^{j} H_{d R}^{i}\left(B . G L_{l} / W_{n}(k)\right) .
\end{aligned}
$$

Moreover, since we can lift Frobenius to each $G L_{j} / W_{n}(k)$, we compute that

$$
H_{\mathrm{cr}}^{i}\left(B . G L_{l} / W_{n}(k), J_{B . G L_{l} / W_{n}(k)}^{<j>} \stackrel{\sim}{\rightarrow} H_{\mathrm{cr}}^{i}\left(B . G L_{l} / W_{n}(k), J_{B . G L_{l} / W_{n}(k)}^{[j]}\right) .\right.
$$

It follows that

$$
\begin{aligned}
& H^{2 i}\left(B . G L_{l} / W(k), S_{n}(i)\right) \stackrel{\sim}{\rightarrow}\left(F^{i} H_{d R}^{2 i}\left(B . G L_{l} / W_{n}(k)\right)\right)^{\phi_{r}=1} \\
& =\left(F^{i} H_{d R}^{2 i}\left(B . G L_{l} / W_{n}(k)\right)\right)^{\phi=p^{r}} \stackrel{\sim}{\leftarrow} H^{2 i}\left(B . G L_{l} / W(k), S_{n}^{\prime}(i)\right) .
\end{aligned}
$$

For $l \geq i$, we define

$$
C_{i, l}^{\mathrm{syn}}=x_{i} \in H^{2 i}\left(B . G L_{l} / W(k), S_{n}(i)\right), \quad C_{i, l}^{\mathrm{syn}}=x_{i} \in H^{2 i}\left(B . G L_{l} / W(k), S_{n}^{\prime}(i)\right) .
$$

By construction these classes are compatible with the crystalline classes. We note that both classes $x_{1} \in H^{2}\left(B . G L_{l} / W(k), S_{n}(1)\right)=H^{2}\left(B . G L_{l} / W(k), S_{n}^{\prime}(1)\right)$ have a direct definition via Kato's $\left[16\right.$, I.3] symbol map $c_{1}^{\text {syn }}: \mathcal{O}_{X}^{*}[-1] \rightarrow i_{*} \mathcal{O}_{X_{n+1}^{*}}^{*}[-1] \rightarrow$ $i_{*} S_{n}(1)$. 
The classes $C_{i, l}^{\text {syn }} \in H^{2 i}\left(B . G L_{l} / W(k), S_{n}(i)\right)$ yield compatible universal classes (see [13, p. 221]) $C_{i, l}^{\mathrm{syn}} \in H^{2 i}\left(X, G L_{l}\left(\mathcal{O}_{X}\right), S_{n}(i)\right)$, hence a natural map of pointed simplicial sheaves on $X, C_{i}^{\mathrm{syn}}: B . G L\left(\mathcal{O}_{X}\right) \rightarrow \mathcal{K}\left(2 i, \tilde{S}_{n}(i)_{X}\right)$, where $\mathcal{K}$ is the DoldPuppe functor of $\tau_{\geq 0} \tilde{S}_{n}(i)_{X}[2 i]$ and $\tilde{S}_{n}(i)_{X}$ is an injective resolution of $S_{n}(i)_{X}$. The characteristic classes $c_{i, j}^{\text {syn }}, j \geq 2$, are now defined [13,2.22] as the composition

$$
\begin{aligned}
K_{j}\left(X, \mathbf{Z} / p^{n}\right) & \rightarrow H^{-j}\left(X, \mathbf{Z} \times B . G L\left(\mathcal{O}_{X}\right)^{+}, \mathbf{Z} / p^{n}\right) \rightarrow H^{-j}\left(X, B . G L\left(\mathcal{O}_{X}\right)^{+}, \mathbf{Z} / p^{n}\right) \\
& \stackrel{C_{i}^{\text {syn }}}{\longrightarrow} H^{-j}\left(X, \mathcal{K}\left(2 i, \tilde{S}_{n}(i)_{X}\right), \mathbf{Z} / p^{n}\right) \stackrel{f}{\rightarrow} H^{2 i-j}\left(X, S_{n}(i)\right),
\end{aligned}
$$

where $B . G L\left(\mathcal{O}_{X}\right)^{+}$is the (pointed) simplicial sheaf on $X$ associated to the +construction $[28,4.2]$. Here, for a (pointed) simplicial sheaf $\mathcal{E}$. on $X, H^{-j}(X, \mathcal{E}$, $\left.\mathbf{Z} / p^{n}\right)=\pi_{j}\left(R \Gamma(X, \mathcal{E}\right.$. $\left.), \mathbf{Z} / p^{n}\right)$ is the generalized sheaf cohomology of $\mathcal{E}$. [13, 1.7]: if we let $\mathcal{P}_{X}^{j}$ denote the constant sheaf of j-dimensional mod $p^{n}$ Moore spaces, then $H^{-j}\left(X, \mathcal{E} ., \mathbf{Z} / p^{n}\right)=\left[\mathcal{P}_{X}^{j}, \mathcal{E}\right.$.], where, for two pointed simplicial sheaves $\mathcal{F}$., $\mathcal{F}^{\prime}$. on $X,\left[\mathcal{F}\right.$., $\mathcal{F}^{\prime}$. denotes the morphisms from $\mathcal{F}$. to $\mathcal{F}^{\prime}$. in the homotopy category. The map $f$ is defined as the composition

$$
\begin{aligned}
H^{-j}\left(X, \mathcal{K}\left(2 i, \tilde{S}_{n}(i)_{X}\right), \mathbf{Z} / p^{n}\right) & \left.\left.=\pi_{j}\left(\mathcal{K}\left(2 i, \tilde{S}_{n}(i)(X)\right), \mathbf{Z} / p^{n}\right)\right) \stackrel{h_{j}}{\rightarrow} H_{j}\left(\mathcal{K}\left(2 i, \tilde{S}_{n}(i)(X)\right), \mathbf{Z} / p^{n}\right)\right) \\
& \rightarrow H_{j}\left(\tilde{S}_{n}(i)(X)[2 i]\right)=H^{2 i-j}\left(X, S_{n}(i)\right),
\end{aligned}
$$

where $h_{j}$ is the Hurewicz morphism.

This gives mod $p^{n}$ Chern classes in $H^{*}\left(X, S_{n}(*)\right)$. Those in $H^{*}\left(X, S_{n}^{\prime}(*)\right)$ and the integral ones are defined in an analogous way.

Lemma 2.6. ([25, Lemma 2.1]) Assume $X$ to be regular. Then

(1) the integral Chern class map $c_{i, j}^{\text {syn }}$, for $j>0$, is a group homomorphism, and $c_{i, j}^{\mathrm{syn}}$ restricts to zero on $F_{\gamma}^{i+1} K_{j}(X), j \geq 0$;

(2) for $j \geq 2$, the mod $p^{n}$ Chern class map $c_{i, j}^{\mathrm{syn}}$ is a group homomorphism and it restricts to zero on $F_{\gamma}^{i+1} K_{j}\left(X, \mathbf{Z} / p^{n}\right)$ unless $j=2$ and $p=2$.

The two types of syntomic Chern classes desribed above are related.

Lemma 2.7. For $j \geq 2$, we have the following commutative diagram

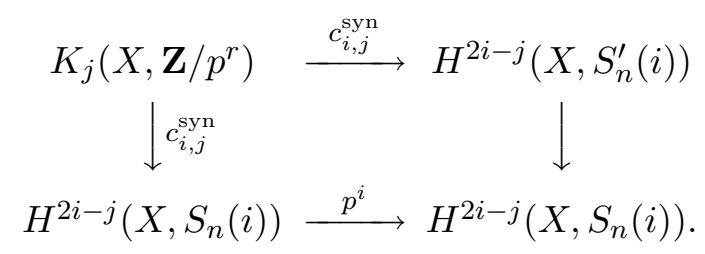


Proof. It is clear from the construction of higher Chern classes recalled above that it suffices to show that $p^{i} C_{i}^{\prime}=C_{i}^{\text {syn }}$, where we wrote $C_{i}^{\prime}$ for the universal Chern class $C_{i}^{\text {syn }} \in H^{2 i}\left(B . G L / W(k), S_{n}^{\prime}(i)\right)$. But for $l \geq i$, both $C_{i, l}^{\prime}$ and $C_{i, l}^{\text {syn }}$ correspond to the class $x_{i} \in F^{i} H_{d R}^{2 i}\left(B . G L_{l} / W_{n}(k)\right)$. The morphism $S_{n}^{\prime}(i) \rightarrow$ $S_{n}(i)$ induces multiplication by $p^{i}$ on $F^{i} H_{d R}^{2 i}\left(B . G L_{l} / W_{n}(k)\right)$. The lemma follows.

Recall that, similarly, we have the étale Chern class maps

$$
c_{i, j}^{\text {ét }}: K_{j}(X) \rightarrow H^{2 i-j}\left(X, \mathbf{Z} / p^{n}(i)\right), \quad c_{i, j}^{\text {ét }}: K_{j}\left(X, \mathbf{Z} / p^{n}\right) \rightarrow H_{\mathrm{cr}}^{2 i-j}\left(X, \mathbf{Z} / p^{n}(i)\right) .
$$

They have analogous properties to those of the syntomic Chern classes.

2.4. $p$-adic comparison theorems. We will review now the statements of $p$ adic comparison theorems.

Conjecture 2.8. (Integral Crystalline conjecture) Let $X$ be smooth and proper scheme over $W(k)$. For $p-2 \geq r \geq i$, there exists a natural Galois equivariant period isomorphism

$$
\alpha_{i, r}: H^{i}\left(X_{\bar{K}}, \mathbf{Z} / p^{n}(r)\right) \simeq F^{r}\left(H_{\mathrm{cr}}^{i}(X / W(k)) \otimes B_{\mathrm{cr}, n}^{+}\right)^{\phi_{r}=1}
$$

This integral form of the Crystalline conjecture was proved by Fontaine-Messing and Kato-Messing [11], [19], by Faltings [6], and by Niziol [23]. The precise conditions on $r$ and $i$ in these proofs vary. Since, by Lemma $2.4, H^{i}\left(X_{\bar{V}}, S_{n}(r)\right) \stackrel{\sim}{\rightarrow}$ $F^{r}\left(H_{\mathrm{cr}}^{i}\left(X / W(k), \mathcal{O}_{X / W(k)} / p^{n}\right) \otimes B_{\mathrm{cr}, n}^{+}\right)^{\phi_{r}=1}$, we will identify integral crystalline period morphisms with isomorphisms

$$
\alpha_{i, r}: H^{i}\left(X_{\bar{K}}, \mathbf{Z} / p^{n}(r)\right) \simeq H^{i}\left(X_{\bar{V}}, S_{n}(r)\right) .
$$

Conjecture 2.9. (Rational Crystalline conjecture) Let $X$ be a proper smooth $V$ scheme. There exists a natural $B_{\mathrm{cr}}$-linear Galois equivariant period isomorphism

$$
\alpha_{i}: H^{i}\left(X_{\bar{K}}, \mathbf{Q}_{p}\right) \otimes_{\mathbf{Q}_{p}} B_{\mathrm{cr}} \simeq H_{\mathrm{cr}}^{i}\left(X_{0} / W(k)\right) \otimes_{W(k)} B_{\mathrm{cr}}
$$

that is compatible with Frobenius and, after extension to $B_{d R}$, induces an isomorphism on filtrations.

For any $r \geq 0$ and a period isomorphism $\alpha_{i}$ as above, define

$$
\alpha_{i, r}: H^{i}\left(X_{\bar{K}}, \mathbf{Q}_{p}(r)\right) \otimes_{\mathbf{Q}_{p}} B_{\mathrm{cr}} \rightarrow H_{\mathrm{cr}}^{i}\left(X_{0} / W(k)\right) \otimes_{W(k)} B_{\mathrm{cr}}\{-r\}
$$


as $\alpha_{i, r}:=t^{r} \alpha_{i} \zeta^{(-r)}$. Here $\{-r\}$ refers to twisting the Frobenius and the filtration. The map $\alpha_{i, r}$ is an isomorphism. It follows that we can recover étale cohomology with the Galois action from the crystalline cohomology:

$\alpha_{i, r}: H^{i}\left(X_{\bar{K}}, \mathbf{Q}_{p}(r)\right) \simeq\left(H_{\mathrm{cr}}^{i}\left(X_{0} / W(k)\right) \otimes_{W(k)} B_{\mathrm{cr}}\right)^{\phi=p^{r}} \cap F^{r}\left(H_{d R}^{i}\left(X_{K}\right) \otimes_{K} B_{d R}\right)$.

For $r \geq i$, by Lemma 2.4, the right hand side is canonically isomorphic to $\mathbf{Q} \otimes \operatorname{proj} \lim _{n} H^{i}\left(X_{\bar{V}}, S_{n}^{\prime}(r)\right)$. Hence we can and will identify rational crystalline period morphisms for $r \geq i$ with isomorphisms

$$
\alpha_{i, r}: H^{i}\left(X_{\bar{K}}, \mathbf{Q}_{p}(r)\right) \simeq \mathbf{Q} \otimes \underset{n}{\operatorname{proj}} \lim H^{i}\left(X_{\bar{V}}, S_{n}^{\prime}(r)\right) .
$$

The Rational Crystalline conjecture was proved by Faltings [6], Niziol [23], and Tsuji [31].

Conjecture 2.10. (Semistable conjecture) Let $X^{\times}$be a proper, log-smooth, vertical, fine and saturated $V^{\times}$-scheme with Cartier type reduction. There exists a natural $B_{\mathrm{st}}$-linear Galois equivariant period isomorphism

$$
\alpha_{i}: H^{i}\left(X_{\bar{K}}, \mathbf{Q}_{p}\right) \otimes_{\mathbf{Q}_{p}} B_{\mathrm{st}} \simeq H_{\mathrm{cr}}^{i}\left(X_{0}^{\times} / W(k)^{0}\right) \otimes_{W(k)} B_{\mathrm{st}}
$$

that preserves the Frobenius and the monodromy operators, and, after extension to $B_{d R}$, induces an isomorphism of filtrations.

The Cartier type condition on the special fiber is equivalent to the special fiber being reduced. It is used to assure that the Frobenius induces an isomorphism on crystalline cohomology and that crystalline cohomology satisfies Poincaré duality.

For any $r \geq 0$ and a period isomorphism $\alpha_{i}$ as above, define

$$
\alpha_{i, r}: H^{i}\left(X_{\bar{K}}, \mathbf{Q}_{p}(r)\right) \otimes_{\mathbf{Q}_{p}} B_{\mathrm{st}} \rightarrow H_{\mathrm{cr}}^{i}\left(X_{0}^{\times} / W(k)^{0}\right) \otimes_{W(k)} B_{\mathrm{st}}\{-r\}
$$

as $\alpha_{i, r}:=t^{r} \alpha_{i} \zeta^{(-r)}$. The map $\alpha_{i, r}$ is an isomorphism. It follows that we can recover étale cohomology with the Galois action from the log-crystalline cohomology:

$H^{*}\left(X_{\bar{K}}, \mathbf{Q}_{p}(r)\right) \simeq\left(H_{\mathrm{cr}}^{*}\left(X_{0}^{\times} / W(k)^{0}\right) \otimes_{W(k)} B_{\mathrm{st}}\right)^{N=0, \phi=p^{r}} \cap F^{r}\left(B_{d R} \otimes_{K} H_{d R}^{*}\left(X_{K} / K\right)\right)$.

For $r \geq i$, by Lemma 2.5, the right hand side is canonically isomorphic to $\mathbf{Q} \otimes \operatorname{proj} \lim _{n} H^{i}\left(X_{\bar{V}}^{\times}, S_{n}^{\prime}(r)\right)$. Hence we can and will identify rational semistable period morphisms for $r \geq i$ with isomorphisms

$$
\alpha_{i, r}: H^{i}\left(X_{\bar{K}}, \mathbf{Q}_{p}(r)\right) \simeq \mathbf{Q} \otimes \underset{n}{\operatorname{proj}} \lim H^{i}\left(X_{\bar{V}}^{\times}, S_{n}^{\prime}(r)\right) .
$$


The Rational Semistable conjecture was first proved by Kato [18] and Tsuji [31] for the schemes with semistable reduction, and later by Faltings [7] and Niziol [25] in general. Here a flat scheme $X$ over $V$ with smooth generic fiber is said to have semistable reduction if it is regular and the special fiber is a divisor with normal crossings.

The above formulation of the Semistable conjecture is due to Fontaine and Jannsen. It was an observation of Faltings [7] that this conjecture can be reformulated in the following way.

Conjecture 2.11. (Semistable conjecture) Let $X^{\times}$be a proper, log-smooth, vertical, fine and saturated $V^{\times}$-scheme with Cartier type reduction. There exists a natural $B_{\mathrm{cr}}$-linear Galois equivariant period isomorphism

$$
\alpha_{i}: H^{i}\left(X_{\bar{K}}, \mathbf{Q}_{p}\right) \otimes_{\mathbf{Q}_{p}} B_{\mathrm{cr}} \simeq H_{\mathrm{cr}}^{i}\left(X^{\times} / R_{V}^{\times}\right) \otimes_{R_{V}} B_{\mathrm{cr}}
$$

that preserves the Frobenius and filtrations.

Here the group $H_{\mathrm{cr}}^{i}\left(X^{\times} / R_{V}^{\times}\right) \otimes_{R_{V}} B_{\mathrm{cr}}^{+}[1 / p]$ is defined by the map $R_{V} \rightarrow B_{\mathrm{cr}}^{+}$, $X \mapsto[\pi]$, and the Galois action of $\sigma \in G_{K}$ is defined as $\sigma \mapsto \exp (\beta(\sigma) N) \otimes \sigma$ (check below for an explanation of that). We recover the étale cohomology by taking Frobenius invariants

$$
\alpha_{i}: H^{i}\left(X_{\bar{K}}, \mathbf{Q}_{p}\right) \simeq F^{0}\left(H_{\mathrm{cr}}^{i}\left(X^{\times} / R_{V}^{\times}\right) \otimes_{R_{V}} B_{\mathrm{cr}}\right)^{\phi=1} .
$$

Proposition 2.12. If period morphisms are compatible with Chern classes of vector bundles, then the above version of the Semistable conjecture is equivalent to the one of Fontaine-Jannsen.

Proof. To see that, recall that $H_{\mathrm{cr}}^{i}\left(X_{n}^{\times} / R_{V, n}^{\times}\right)$is an $R_{V, n}^{\times}$-module equipped with a quasi-nilpotent integrable connection, a descending filtration, and a horizontal Frobenius. The connection satisfies Griffiths transversality. It follows [31, Prop. 1.6.15] that any two pullbacks of $H_{\mathrm{cr}}^{i}\left(X_{n}^{\times} / R_{V, n}^{\times}\right)$to $\widehat{B}_{\mathrm{st}, n}^{+}$are canonically isomorphic. In particular, we have a $\widehat{B}_{\mathrm{st}, n}^{+}$-linear horizontal filtered compatible with Frobenius isomorphism

$$
\begin{aligned}
& \omega: \quad H_{\mathrm{cr}}^{i}\left(X_{n}^{\times} / R_{V, n}^{\times}\right) \otimes_{R_{V, n}^{\times}, \pi} \widehat{B}_{\mathrm{st}, n}^{+} \stackrel{\sim}{\longrightarrow} H_{\mathrm{cr}}^{i}\left(X_{n}^{\times} / R_{V, n}^{\times}\right) \otimes_{R_{V, n}^{\times}} \widehat{B}_{\mathrm{st}, n}^{+}, \\
& x \otimes 1 \mapsto \Sigma_{i \geq 0} \Pi_{0 \leq j<i}(N-j)(x)\left(v_{[\pi]}^{-1}-1\right)^{[i]}=\exp \left(N \otimes u_{[\pi]}\right),
\end{aligned}
$$


where the first pullback is via $X \mapsto[\pi]$ and the second via the canonical map $R_{V, n}^{\times} \rightarrow \widehat{B}_{\mathrm{st}, n}^{+}$. Similarly, for $\sigma \in G_{K}$, we can compare the pullbacks to $\widehat{B}_{\mathrm{st}, n}^{+}$ via $X \mapsto[\pi]$ and via $X \mapsto \sigma([\pi])=\beta(\sigma)[\pi]$ and get the Galois action on $H_{\mathrm{cr}}^{i}\left(X_{n}^{\times} / R_{V, n}^{\times}\right) \otimes_{R_{V, n}^{\times}, \pi} \widehat{B}_{\mathrm{st}, n}^{+}$as $\exp (\log (\beta(\sigma)) N) \otimes \sigma$. The isomorphism $\omega$ is then Galois equivariant.

Taking now the subspaces of both sides in (2.5) where the monodromy acts trivially and using the isomorphism

$$
H_{\mathrm{cr}}^{i}\left(X^{\times} / R_{V, n}^{\times}\right) \otimes_{R_{V, n}} B_{\mathrm{cr}, n}^{+} \simeq\left(H_{\mathrm{cr}}^{i}\left(X_{n}^{\times} / R_{V, n}^{\times}\right) \otimes_{R_{V, n}^{\times}, \pi} \widehat{B}_{\mathrm{st}, n}^{+}\right)^{N=0}
$$

(notice that the monodromy is trivial on the left hand side because $\operatorname{dlog}([\pi])$ is zero in $\left.\Omega_{B_{\mathrm{cr}, n}^{+} \otimes W_{n}(k)[X]^{\times} / B_{\mathrm{cr}, n}^{+, \times}}^{1}\right)$ we get a filtered, Galois equivariant, compatible with Frobenius isomorphism

$$
\omega: H_{\mathrm{cr}}^{i}\left(X^{\times} / R_{V, n}^{\times}\right) \otimes_{R_{V, n}} B_{\mathrm{cr}, n}^{+} \stackrel{\sim}{\rightarrow}\left(H_{\mathrm{cr}}^{i}\left(X_{n}^{\times} / R_{V, n}^{\times}\right) \otimes_{R_{V, n}^{\times}} \widehat{B}_{\mathrm{st}, n}^{+}\right)^{N=0} .
$$

We can use the last isomorphism to pass between Faltings and FontaineJannsen version of the Semistable conjecture. Consider the following commutative diagram.

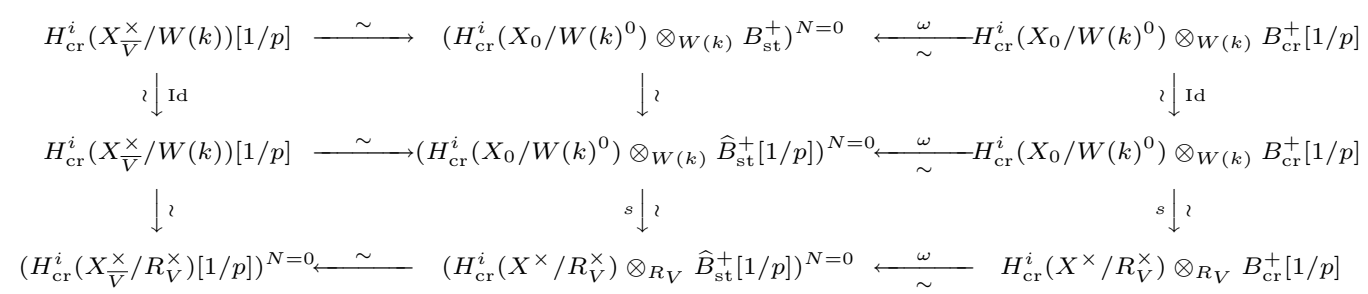

Here, as above, we equip $H_{\mathrm{cr}}^{i}\left(X_{0} / W(k)^{0}\right) \otimes B_{\mathrm{cr}}^{+}[1 / p]$ with the Galois action given by $\exp (\log (\beta(\sigma)) N) \otimes \sigma$ for $\sigma \in G_{K}$. The $B_{\text {cr }}^{+}[1 / p]$-linear morphism

$$
\omega: H_{\mathrm{cr}}^{i}\left(X_{0} / W(k)^{0}\right) \otimes B_{\mathrm{cr}}^{+}[1 / p] \rightarrow\left(H_{\mathrm{cr}}^{i}\left(X_{0} / W(k)^{0}\right) \otimes B_{\mathrm{st}}^{+}\right)^{N=0}
$$

is given by $\exp \left(N \otimes u_{[\pi]}\right)$. It commutes with Galois action and Frobenius.

Now, having the Faltings-type period isomorphism

$$
\alpha_{i}: H^{i}\left(X_{\bar{K}}, \mathbf{Q}_{p}\right) \otimes_{\mathbf{Q}_{p}} B_{\mathrm{cr}} \simeq H_{\mathrm{cr}}^{i}\left(X^{\times} / R_{V}^{\times}\right) \otimes_{R_{V}} B_{\mathrm{cr}}
$$

we can compose it with the section $s$ and the map $\omega$ and get an isomorphism

$$
\alpha_{i}: \quad H^{i}\left(X_{\bar{K}}, \mathbf{Q}_{p}\right) \otimes_{\mathbf{Q}_{p}} B_{\mathrm{cr}} \simeq\left(H_{\mathrm{cr}}^{i}\left(X_{0} / W(k)^{0}\right) \otimes_{W(k)} B_{\mathrm{st}}\right)^{N=0} .
$$

Tensoring with $B_{\mathrm{st}}$ then yields an isomorphism

$$
\tilde{\alpha}_{i}: H^{i}\left(X_{\bar{K}}, \mathbf{Q}_{p}\right) \otimes_{\mathbf{Q}_{p}} B_{\mathrm{st}} \simeq H_{\mathrm{cr}}^{i}\left(X_{0} / W(k)^{0}\right) \otimes_{W(k)} B_{\mathrm{st}}
$$


It is compatible with Galois action, Frobenius (since the maps $s$ and $\omega$ are), and monodromy. We claim that, extended to $B_{d R}$, it induces an isomorphism on filtrations. Indeed, Faltings-type period isomorphism $\alpha_{i}$ is strict for filtrations. We first check that this implies that $\tilde{\alpha}_{i}$ is compatible with filtrations. For that recall that the map

$$
H_{\mathrm{cr}}^{i}\left(X_{0} / W(k)^{0}\right) \otimes_{W(k)} B_{\mathrm{st}} \rightarrow H_{d R}^{i}\left(X_{K} / K\right) \otimes B_{d R}
$$

is induced by the composition

$$
\begin{aligned}
& H_{\mathrm{cr}}^{i}\left(X_{0} / W(k)^{0}\right) \otimes_{W(k)} B_{\mathrm{st}}^{+} \rightarrow H_{\mathrm{cr}}^{i}\left(X_{0} / W(k)^{0}\right) \otimes_{W(k)} \widehat{B}_{\mathrm{st}}^{+} \\
& \stackrel{\omega}{\rightarrow} H_{\mathrm{cr}}^{i}\left(X^{\times} / R_{V}^{\times}\right) \otimes_{R_{V}} \widehat{B}_{\mathrm{st}}^{+}[1 / p] \stackrel{\sim}{\rightarrow} H^{i}\left(X_{\bar{V}}^{\times} / R_{V}^{\times}\right)[1 / p] \\
& \rightarrow \underset{s}{\operatorname{proj}} \lim H_{\mathrm{cr}}^{i}\left(X^{\times} / V_{V}^{\times}, \mathcal{O} / J^{[s]}\right)[1 / p] \leftarrow H_{d R}^{i}\left(X_{K} / K\right) \otimes B_{d R}^{+}
\end{aligned}
$$

Chasing the above diagram we conclude that the map

$$
\tilde{\alpha}_{i}: H^{i}\left(X_{\bar{K}}, \mathbf{Q}_{p}\right) \otimes_{\mathbf{Q}_{p}} B_{d R} \simeq H_{d R}^{i}\left(X_{K} / K\right) \otimes_{W(k)} B_{d R}
$$

is the composition of Faltings-type period map $\alpha_{i}$ and the map

$$
\begin{gathered}
H_{\mathrm{cr}}^{i}\left(X^{\times} / R_{V}^{\times}\right) \otimes_{R_{V}} B_{\mathrm{cr}}^{+}[1 / p] \stackrel{\omega}{\rightarrow} H_{\mathrm{cr}}^{i}\left(X^{\times} / R_{V}^{\times}\right) \otimes_{R_{V}} \widehat{B}_{\mathrm{st}}^{+}[1 / p] \rightarrow H^{i}\left(X_{\bar{V}}^{\times} / R_{V}^{\times}\right)[1 / p] \\
\rightarrow \operatorname{proj} \lim _{s} H_{\mathrm{cr}}^{i}\left(X^{\times} / V_{V}^{\times}, \mathcal{O} / J^{[s]}\right)[1 / p] \stackrel{\sim}{\leftarrow} H_{d R}^{i}\left(X_{K} / K\right) \otimes B_{d R}^{+}
\end{gathered}
$$

Since all the maps in the above are compatible with filtrations our claim follows.

To check that the map $\tilde{\alpha}_{i}$ is strict for filtrations it suffices now to check injectivity on the associated gradings. By Poincaré duality in étale cohomology and Serre duality this can be reduced (see the proof of Theorem 4.10.2 in [31]) to checking that the map

$$
\tilde{\alpha}_{2 d}: \quad \operatorname{gr}^{0}\left(H^{2 d}\left(X_{\bar{K}}, \mathbf{Q}_{p}\right) \otimes_{\mathbf{Q}_{p}} B_{d R}\right) \rightarrow \operatorname{gr}^{0}\left(H_{d R}^{2 d}\left(X_{K} / K\right) \otimes_{W(k)} B_{d R}\right),
$$

where $d$ denotes the dimension of $X_{K}$, is an isomorphism. By a standard argument (see [11, III 6.3], [31, Lemma 4.10.3]) blowing-up reduces this to checking compatibility of $\tilde{\alpha}_{i}$ with Chern classes of line bundles. By assumption, Faltings-type period map is compatible with the crystalline classes $c_{1}^{\text {cr }}(\mathcal{L}) \in$ $F^{1} H_{\mathrm{cr}}^{2}\left(X^{\times} / R_{V}^{\times}\right)$and the étale classes $c_{1}^{\text {ét }}(\mathcal{L}) \in H^{2 d}\left(X_{\bar{K}}, \mathbf{Q}_{p}\right) \otimes F^{1} B_{\mathrm{cr}}$ for line bundles $\mathcal{L}$ on $X$. Since $c_{1}^{\mathrm{cr}}(\mathcal{L})$ comes from $H_{\mathrm{cr}}^{2}(X / W(k))$ it is invariant under the monodromy hence invariant under the map $\omega$. Since $c_{1}^{\mathrm{cr}}(\mathcal{L})$ maps to $c_{1}^{d R}\left(\mathcal{L}_{K}\right) \in H_{\mathrm{cr}}^{2}\left(X^{\times} / V^{\times}\right)[1 / p] \simeq H_{d R}^{2}\left(X_{K} / K\right)$, we are done. 
We have shown that Faltings version of the Semistable conjecture implies the one of Fontaine-Jannsen (assuming compatibility of both period maps with Chern classes of vector bundles). The above argument can be basically inverted giving the claimed equivalence of both versions of the Semistable conjecture. It is interesting to note that in passing between the two versions we ended up working with the ring $\widehat{B}_{\mathrm{st}}^{+}$and Galois representations that are $\widehat{B}_{\mathrm{st}}^{+}[1 / p, 1 / t]$-admissible. See [2] for a discussion of that subject. In particular, the fact [2, Theorem 7.1] that the category of $\widehat{B}_{\mathrm{st}}^{+}[1 / p, 1 / t]$-admissible representations is equivalent to the category of semistable representation, i.e., $B_{\mathrm{st}}$-admissible representations.

\section{MOtIVIC PERIOD MORPHISMS AND UNIQUENESS CRITERIA}

In this section, we will first review our $p$-adic comparison morphisms [23], [25]. Then we will state uniqueness criteria for $p$-adic period morphisms that follow from our constructions. Assume first that $X$ is a smooth scheme over $W(k)$. The $p$-adic comparison map in this situation is defined by the following diagram

$$
\begin{array}{cc}
F_{\gamma}^{r} / F_{\gamma}^{r+1} K_{2 r-i}\left(X_{\bar{V}}, \mathbf{Z} / p^{n}\right) \stackrel{j^{*}}{\longrightarrow} F_{\gamma}^{r} / F_{\gamma}^{r+1} K_{2 r-i}\left(X_{\bar{K}}, \mathbf{Z} / p^{n}\right) \\
\downarrow c_{r, 2 r-i}^{\mathrm{syn}} & \downarrow c_{r, 2 r-i}^{\text {et }} \\
H^{i}\left(X_{\bar{V}}, S_{n}(r)\right) & H^{i}\left(X_{\bar{K}}, \mathbf{Z} / p^{n}(r)\right),
\end{array}
$$

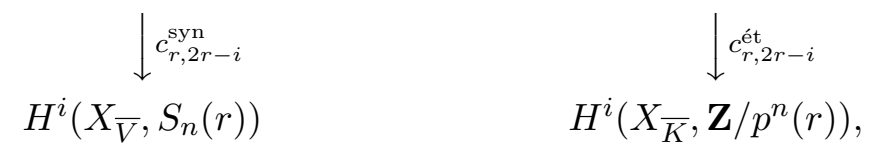

where $j^{*}$ is the restriction map. We have proved in [23, Lemma 3.1] that the map

$$
K_{m}\left(X_{\bar{V}}, \mathbf{Z} / p^{n}\right) \stackrel{j^{*}}{\longrightarrow} K_{m}\left(X_{\bar{K}}, \mathbf{Z} / p^{n}\right)
$$

is an isomorphism for all $m$. This is so because the kernel and cokernel of the localization map $j^{*}$ is controlled by the $K$-groups of the special fibers and, since we work with $K$-theory $\bmod p^{n}$, those are killed by some ramified extensions of $W(k)$.

Let $d$ be the dimension of $X_{K}$. Assume that $p-2 \geq \max \{r, d+2 r-i\}$ and $2 r-i \geq \max \{2 d+1,2\}$. Under these assumptions the étale Chern classes $c_{r, 2 r-i}^{\text {ét }}$ are isomorphisms. More precisely, we have the following proposition [25, Proposition 3.2].

Proposition 3.1. There exists an integer $T(d, r, j)$ depending only on $d, r, j$ such that, the kernel and cokernel of the Chern classes

$$
c_{i j}^{\text {ét }}: F_{\gamma}^{r} / F_{\gamma}^{r+1} K_{j}\left(X_{\bar{K}}, \mathbf{Z} / p^{n}\right) \rightarrow H^{2 r-j}\left(X_{\bar{K}}, \mathbf{Z} / p^{n}(r)\right)
$$


are annihilated by $T(d, r, j)$. An odd prime $p$ divides $T(d, r, j)$ if and only if $p \leq d+j+1$.

This proposition is a $K$-theory version of the following theorem of Suslin [29].

Theorem 3.2. For $r \geq d$, there is an isomorphism

$$
H_{\text {Zar }}^{j}\left(X_{\bar{K}}, \mathbf{Z} / p^{n}(r)\right) \stackrel{\sim}{\rightarrow} H^{j}\left(X_{\bar{K}}, \mathbf{Z} / p^{n}(r)\right),
$$

where $H_{\mathrm{Zar}}^{j}\left(X_{\bar{K}}, \mathbf{Z} / p^{n}(r)\right)$ are the Bloch higher Chow groups [1] appropriately reindexed.

We know that the Bloch higher Chow groups $H_{\mathrm{Zar}}^{j}\left(X_{\bar{K}}, \mathbf{Z} / p^{n}(r)\right)$ and the graded pieces of $K$-theory $F_{\gamma}^{r} / F_{\gamma}^{r+1} K_{j}\left(X_{\bar{K}}, \mathbf{Z} / p^{n}\right)$ are isomorphic modulo wellcontrolled torsion. The integer $T(d, r, j)$ appearing in the above proposition is basically the one controlling that torsion.

Our $p$-adic period morphism

$$
\alpha_{i, r}^{N}: H^{i}\left(X_{\bar{K}}, \mathbf{Z} / p^{n}(r)\right) \rightarrow H^{i}\left(X_{\bar{V}}, S_{n}(r)\right)
$$

is defined as the composition $\alpha_{i, r}^{N}: c_{r, 2 r-i}^{\text {syn }}\left(j^{*}\right)^{-1}\left(c_{r, 2 r-i}^{\text {et }}\right)^{-1}$. It is clearly Galois equivariant.

Theorem 3.3. (Niziol [23, Theorem 4.1]) For $p-2 \geq \max \{r, d+2 r-i, 2 r+i-d\}$ and $\min \{2 r-i, 2 r-2 d+i\} \geq \max \{2 d+1,2\}$, the morphism

$$
\alpha_{i, r}^{N}: H^{i}\left(X_{\bar{K}}, \mathbf{Z} / p^{n}(r)\right) \stackrel{\sim}{\rightarrow} H^{i}\left(X_{\bar{V}}, S_{n}(r)\right)
$$

is an isomorphism.

The following uniqueness criterium follows immediately.

Theorem 3.4. For $p-2 \geq \max \{r, d+2 r-i\}$ and $2 r-i \geq \max \{2 d+1,2\}$, there exists a unique crystalline period morphism

$$
\alpha_{i, r}: H^{i}\left(X_{\bar{K}}, \mathbf{Z} / p^{n}(r)\right) \rightarrow H^{i}\left(X_{\bar{V}}, S_{n}(r)\right)
$$

that makes the above diagram commute.

Proof. This is implied by the fact that under the stated assumptions the restriction map $j^{*}$ and the étale Chern classes $c_{r, 2 r-i}^{\text {ét }}$ are isomorphisms. 
Remark 3.5. The constants appearing in the above theorems could be improved if instead of Suslin's theorem quoted above we could use the BeilinsonLichtenbaum conjecture

Conjecture 3.6. For $r \geq j$, there is an isomorphism

$$
H_{\mathrm{Zar}}^{j}\left(X_{\bar{K}}, \mathbf{Z} / p^{n}(r)\right) \stackrel{\sim}{\rightarrow} H^{j}\left(X_{\bar{K}}, \mathbf{Z} / p^{n}(r)\right) .
$$

The Beilinson-Lichtenbaum conjecture follows from the Bloch-Kato conjecture that is now proved by Voevodsky [33] for prime $p=2$ (the Milnor conjecture). Voevodsky has recently announced [34] a proof of the Bloch-Kato conjecture for all primes $p$. The interested reader will find more information on the subject of the Bloch-Kato conjecture and its relation to $p$-adic periods in [26].

Let now $X$ be a smooth scheme over $V$. Consider the following diagram

$$
\begin{array}{rr}
\mathbf{Q} \otimes \operatorname{proj} \lim _{n} F_{\gamma}^{r} / F_{\gamma}^{r+1} K_{2 r-i}\left(X_{\bar{V}}, \mathbf{Z} / p^{n}\right) \underset{j^{*}}{\longrightarrow} \mathbf{Q} \otimes \operatorname{proj} \lim _{n} F_{\gamma}^{r} / F_{\gamma}^{r+1} K_{2 r-i}\left(X_{\bar{K}}, \mathbf{Z} / p^{n}\right) \\
\qquad c_{r, 2 r-i}^{\text {syn }} \\
\mathbf{Q} \otimes \operatorname{proj} \lim _{n} H^{i}\left(X_{\bar{V}}, S_{n}^{\prime}(r)\right) & \quad c_{r, 2 r-i}^{\text {ct }} \\
\mathbf{Q} \otimes \operatorname{proj} \lim _{n} H^{i}\left(X_{\bar{K}}, \mathbf{Z} / p^{n}(r)\right) .
\end{array}
$$

As above the restriction map $j^{*}$ is an isomorphism. Let $d$ be the dimension of $X_{K}$. Let $2 r-i \geq \max \{2 d+1,2\}$ and $2 r-i \geq 3$ for $p=2, d=0$. It follows from Proposition 3.1 that the étale Chern classes $c_{r, 2 r-i}^{\text {et }}$ are isomorphisms rationally. Set $H^{i}\left(X_{\bar{V}}, S_{\mathbf{Q}_{p}}^{\prime}(r)\right):=\mathbf{Q} \otimes \operatorname{proj} \lim _{n} H^{i}\left(X_{\bar{V}}, S_{n}^{\prime}(r)\right)$. We define the $p$-adic period morphism

$$
\alpha_{i, r}^{N}: \quad H^{i}\left(X_{\bar{K}}, \mathbf{Q}_{p}(r)\right) \rightarrow H^{i}\left(X_{\bar{V}}, S_{\mathbf{Q}_{p}}^{\prime}(r)\right)
$$

as the composition $\alpha_{i, r}^{N}: c_{r, 2 r-i}^{\mathrm{syn}}\left(j^{*}\right)^{-1}\left(c_{r, 2 r-i}^{\text {ét }}\right)^{-1}$. It is clearly Galois equivariant.

Theorem 3.7. (Niziol [23, Theorem 5.1]) Let $2 r-i \geq \max \{2 d+1,2\}$ and $2 r-i \geq 3$ for $p=2, d=0$. The $p$-adic period morphism

$$
\alpha_{i, r}^{N}: \quad H^{i}\left(X_{\bar{K}}, \mathbf{Q}_{p}(r)\right) \otimes B_{\text {cr }} \stackrel{\sim}{\rightarrow} H^{i}\left(X_{0} / W(k)\right) \otimes B_{\text {cr }}\{-r\}
$$

is an isomorphism. It is compatible with Frobenius, Galois action and, after extension to $B_{d R}$, induces an isomorphism on filtrations.

Again a uniqueness criterium follows immediately. 
Theorem 3.8. Let $2 r-i \geq \max \{2 d+1,2\}$ and $2 r-i \geq 3$ for $p=2, d=0$. There exists a unique crystalline period morphism

$$
\alpha_{i, r}: H^{i}\left(X_{\bar{K}}, \mathbf{Q}_{p}(r)\right) \rightarrow H^{i}\left(X_{\bar{V}}, S_{\mathbf{Q}_{p}}^{\prime}(r)\right)
$$

that makes the above diagram commute.

Proof. Use the fact that the restriction map $j^{*}$ and the étale Chern classes $c_{r, 2 r-i}^{\text {ét }}$ are isomorphisms.

Let now $X^{\times}$be a saturated, $\log$-smooth, vertical $\log$-scheme over $V^{\times}$. Consider the following diagram

$$
\begin{array}{rr}
\mathbf{Q} \otimes \operatorname{proj} \lim _{n} F_{\gamma}^{r} / F_{\gamma}^{r+1} K_{2 r-i}\left(X_{\bar{V}}^{\times}, \mathbf{Z} / p^{n}\right) \stackrel{j^{*}}{\longrightarrow} \mathbf{Q} \otimes \operatorname{proj} \lim _{n} F_{\gamma}^{r} / F_{\gamma}^{r+1} K_{2 r-i}\left(X_{\bar{K}}, \mathbf{Z} / p^{n}\right) \\
\qquad c_{r, 2 r-i}^{\mathrm{syn}} & \downarrow_{r, 2 r-i}^{\text {et }} \\
\mathbf{Q} \otimes \operatorname{proj} \lim _{n} H^{i}\left(X_{\bar{V}}^{\times}, S_{n}^{\prime}(r)\right) & \mathbf{Q} \otimes \operatorname{proj} \lim _{n} H^{i}\left(X_{\bar{K}}, \mathbf{Z} / p^{n}(r)\right) .
\end{array}
$$

Here we wrote $K_{*}\left(X_{\bar{V}}^{\times}, \mathbf{Z} / p^{n}\right)$ for the direct limit

$$
K_{*}\left(X_{\bar{V}}^{\times}, \mathbf{Z} / p^{n}\right):=\operatorname{inj}_{Y^{\times} \rightarrow X_{V_{1}}^{\times}} K_{*}\left(Y, \mathbf{Z} / p^{n}\right)
$$

over log-blow-ups $Y^{\times} \rightarrow X_{V_{1}}^{\times}$of the base changes $X_{V_{1}}^{\times}$of $X^{\times}$to a finite extension $V_{1}$ of $V$. The fact that these log-blow-ups form a cofiltered system follows from [24]. We also show in that paper that (classically) regular log-blowups are cofinal. Hence $\gamma$-filtration is well-defined. The syntomic Chern classes $c_{r, 2 r-i}^{\mathrm{syn}}: K_{2 r-1}\left(X_{\bar{V}}^{\times}, \mathbf{Z} / p^{n}\right) \rightarrow H^{i}\left(X_{\bar{V}}^{\times}, S_{n}^{\prime}(r)\right)$ are defined as the following composition

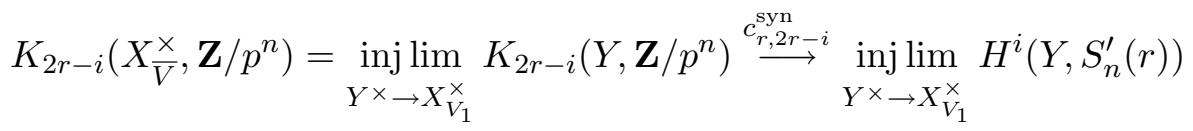

$$
\begin{aligned}
& \rightarrow \operatorname{inj}_{Y^{\times} \rightarrow X_{V_{1}}^{\times}} H^{i}\left(Y^{\times}, S_{n}^{\prime}(r)\right) \stackrel{\sim}{\sim} \operatorname{inj}_{V_{1} \rightarrow V} \lim _{1} H^{i}\left(X_{V_{1}}^{\times}, S_{n}^{\prime}(r)\right)=H^{i}\left(X_{\bar{V}}^{\times}, S_{n}^{\prime}(r)\right),
\end{aligned}
$$

where the last isomorphism follows from [25, Proposition 2.3] (log-blow-ups do not change syntomic cohomology).

Set $H^{i}\left(X_{\bar{V}}^{\times}, S_{\mathbf{Q}_{p}}^{\prime}(r)\right):=\mathbf{Q} \otimes \operatorname{proj} \lim _{n} H^{i}\left(X_{\bar{V}}^{\times}, S_{n}^{\prime}(r)\right)$. Let $d$ denote the dimension of $X_{K}$. Let $2 r-i \geq \max \{2 d+1,2\}$ and $2 r-i \geq 3$ for $p=2, d=0$. Just as above the étale Chern classes are isomorphisms. It turns out that the restriction map $j^{*}$ is an isomorphism as well (although only for high enough $2 r-i$ ). Let $K_{1}$ 
denote a finite extension of $K$ and let $V_{1}$ be its ring of integers. In [25, Lemma 3.5] we have proved the following lemma.

Lemma 3.9. Let $Y$ be a regular flat scheme over $V_{1}$ and let $j: Y_{K_{1}} \hookrightarrow Y$ be the open immersion. Then the restriction map

$$
j^{*}: K_{j}\left(Y, \mathbf{Z} / p^{n}\right) \stackrel{\sim}{\rightarrow} K_{j}\left(Y_{K_{1}}, \mathbf{Z} / p^{n}\right), \quad j>d+1,
$$

is an isomorphism and the induced map

$$
j^{*}: F_{\gamma}^{i} / F_{\gamma}^{i+1} K_{j}\left(Y, \mathbf{Z} / p^{n}\right) \rightarrow F_{\gamma}^{i} / F_{\gamma}^{i+1} K_{j}\left(Y_{K_{1}}, \mathbf{Z} / p^{n}\right), \quad j>d+1
$$

has kernel and cokernel annihilated by integers depending only on the dimension of $Y_{K_{1}}, i$, and $j$.

Via the localization sequence in $K^{\prime}$-theory, this lemma follows from the following vanishing result of Geisser-Levine [12].

Theorem 3.10. Let $Y$ be a nonsingular scheme of dimension d over a perfect field of positive characteristic. Then $K_{j}\left(Y, \mathbf{Z} / p^{n}\right)=0$ for $j>d$.

Since regular schemes are cofinal in the limit defining $K_{2 r-i}\left(X_{\bar{V}}^{\times}, \mathbf{Z} / p^{n}\right)$, the above lemma implies that the restriction map

$\mathbf{Q} \otimes \underset{n}{\operatorname{proj}} \lim F_{\gamma}^{r} / F_{\gamma}^{r+1} K_{2 r-i}\left(X_{\bar{V}}^{\times}, \mathbf{Z} / p^{n}\right) \stackrel{j^{*}}{\rightarrow} \mathbf{Q} \otimes \underset{n}{\operatorname{proj}} \lim F_{\gamma}^{r} / F_{\gamma}^{r+1} K_{2 r-i}\left(X_{\bar{K}}, \mathbf{Z} / p^{n}\right)$ is an isomorphism, as wanted.

A uniqueness criterium follows.

Theorem 3.11. Let $2 r-i \geq \max \{2 d+1,2\}$ and $2 r-i \geq 3$ for $p=2, d=0$. There exists a unique semistable period morphism

$$
\alpha_{i, r}: H^{i}\left(X_{\bar{K}}, \mathbf{Q}_{p}(r)\right) \rightarrow H^{i}\left(X_{\bar{V}}, S_{\mathbf{Q}_{p}}^{\prime}(r)\right)
$$

that makes the above diagram commute.

Proof. Use the fact that the étale Chern classes $c_{r, 2 r-i}^{\text {t }}$ are isomorphisms rationally and that the restriction map $j^{*}$ is an isomorphism.

We define the period morphism

$$
\alpha_{i, r}^{N}: H^{i}\left(X_{\bar{K}}, \mathbf{Q}_{p}(r)\right) \rightarrow H^{i}\left(X_{\bar{V}}, S_{\mathbf{Q}_{p}}^{\prime}(r)\right)
$$

as the composition $\alpha_{i, r}^{N}: c_{r, 2 r-i}^{\mathrm{syn}}\left(j^{*}\right)^{-1}\left(c_{r, 2 r-i}^{\text {ét }}\right)^{-1}$. 
Theorem 3.12. (Niziol [25, Theorem 3.9]) Let $2 r-i \geq \max \{2 d+1,2\}$ and $2 r-i \geq 3$ for $p=2, d=0$. The induced $B_{\mathrm{st}}$-linear period morphism

$$
\alpha_{i, r}^{N}: H^{i}\left(X_{\bar{K}}, \mathbf{Q}_{p}(r)\right) \otimes B_{\mathrm{st}} \stackrel{\sim}{\rightarrow} H^{i}\left(X_{0} / W(k)\right) \otimes B_{\mathrm{st}}\{-r\}
$$

is an isomorphism. It is compatible with Frobenius, monodromy, Galois action, and filtration after passing to $B_{d R}$.

\section{Syntomic Period MORPhisms}

For a scheme $X$ on the small syntomic site of $\operatorname{Spec}(W(k))$, define

$$
\mathcal{O}_{n}^{\mathrm{cr}}(X)=H_{\mathrm{cr}}^{0}\left(X_{n} / W_{n}(k), \mathcal{O}_{X_{n} / W_{n}(k)}\right), \quad \mathcal{J}_{n}^{[r]}(X)=H_{\mathrm{cr}}^{0}\left(X_{n} / W_{n}(k), \mathcal{J}_{X_{n} / W_{n}(k)}^{[r]}\right),
$$

where $\mathcal{O}_{X_{n} / W_{n}(k)}$ is the structure sheaf of the crystalline site, $\mathcal{J}_{X_{n} / W_{n}(k)}=$ $\operatorname{Ker}\left(\mathcal{O}_{X_{n} / W_{n}(k)} \rightarrow \mathcal{O}_{X_{n}}\right)$, and $\mathcal{J}_{X_{n} / W_{n}(k)}^{[r]}$ is its $r^{\prime}$ th divided power of $\mathcal{J}_{X_{n} / W_{n}(k)}$. Set $\mathcal{J}_{X_{n} / W_{n}(k)}^{[r]}=\mathcal{O}_{X_{n} / W_{n}(k)}$ if $r \leq 0$. We know [11, II.1.3] that the presheaves $\mathcal{J}_{n}^{[r]}$ are sheaves, flat over $\mathbf{Z} / p^{n}$, and that $\mathcal{J}_{n+1}^{[r]} \otimes \mathbf{Z} / p^{n} \simeq \mathcal{J}_{n}^{[r]}$. There is a canonical, compatible with Frobenius, and functorial isomorphism

$$
H_{\mathrm{syn}}^{*}\left(X, \mathcal{J}_{n}^{[r]}\right) \simeq H_{\mathrm{cr}}^{*}\left(X_{n} / W_{n}(k), \mathcal{J}_{X_{n} / W_{n}(k)}^{[r]}\right) .
$$

It is easy to see that $\phi\left(\mathcal{J}_{n}^{[r]}\right) \subset p^{r} \mathcal{O}_{n}^{\text {cr }}$ for $0 \leq r \leq p-1$. This fails in general and we modify $\mathcal{J}_{n}^{[r]}$ :

$$
\mathcal{J}_{n}^{<r>}:=\left\{x \in \mathcal{J}_{n+s}^{[r]} \mid \phi(x) \in p^{r} \mathcal{O}_{n+s}^{\text {cr }}\right\} / p^{n},
$$

for some $s \geq r$. This definition is independent of $s$. We check that $\mathcal{J}_{n}^{<r>}$ is flat over $\mathbf{Z} / p^{n}$ and $\mathcal{J}_{n+1}^{<r>} \otimes \mathbf{Z} / p^{n} \simeq \mathcal{J}_{n}^{<r>}$. This allows us to define the divided Frobenius $\phi_{r}=" \phi / p^{r} ": \mathcal{J}_{n}^{<r>} \rightarrow \mathcal{O}_{n}^{\text {cr }}$. Set $\mathcal{S}_{n}(r):=\operatorname{Ker}\left(\mathcal{J}_{n}^{<r>} \stackrel{\phi_{r}-1}{\longrightarrow} \mathcal{O}_{n}^{\text {cr }}\right)$. In the same way we can define syntomic sheaves $\mathcal{S}_{n}(r)$ on $X_{m \text {,syn }}$ for $m \geq n$. Abusing notation, we have $\mathcal{S}_{n}(r)=i_{*} \mathcal{S}_{n}(r)$ for the natural map $i: X_{m \text {,syn }} \rightarrow X_{\text {syn }}$. Since $i_{*}$ is exact, $H_{\mathrm{syn}}^{*}\left(X_{m}, \mathcal{S}_{n}(r)\right)=H_{\mathrm{syn}}^{*}\left(X, \mathcal{S}_{n}(r)\right)$. Because of that we will write $\mathcal{S}_{n}(r)$ for the syntomic sheaves on $X_{m \text {,syn }}$ as well as on $X_{\text {syn }}$. We will also need the " "undivided"' version of syntomic sheaves: $\mathcal{S}_{n}^{\prime}(r):=\operatorname{Ker}\left(\mathcal{J}_{n}^{[r]} \stackrel{\phi-p^{r}}{\longrightarrow} \mathcal{O}_{n}^{\text {cr }}\right)$. There is a natural map $\mathcal{S}_{n}^{\prime}(r) \rightarrow \mathcal{S}_{n}(r)$ whose kernel and cokernel are killed by $p^{r}$. If it does not cause confusion, we will also write $\mathcal{S}_{n}(r), \mathcal{S}_{n}^{\prime}(r)$ for $R \varepsilon_{*} \mathcal{S}_{n}(r)$,

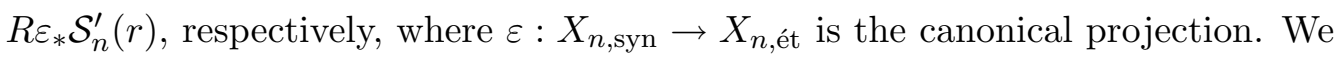
have natural quasi-isomorphisms $R \pi_{*} \mathcal{S}_{n}(r) \simeq S_{n}(r), R \pi_{*} \mathcal{S}_{n}^{\prime}(r) \simeq S_{n}^{\prime}(r)$, where $\pi: X_{\text {ét }} \rightarrow X_{\text {Zar }}$ is the canonical projection. 
Proposition 4.1. ([11, III.1.1]) The following sequence is exact for $r \geq 0$

$$
0 \longrightarrow \mathcal{S}_{n}(r) \longrightarrow \mathcal{J}_{n}^{<r>} \stackrel{\phi_{r}-1}{\longrightarrow} \mathcal{O}_{n}^{\text {cr }} \longrightarrow 0 .
$$

Let $X$ be a smooth scheme over $V$. For $0 \leq r \leq p-2$, there is a natural homomorphism on the étale site of $X_{n}$

$$
\alpha_{r}: R \varepsilon_{*} \mathcal{S}_{n}(r) \rightarrow \tau_{\leq r} i^{*} R j_{*} \mathbf{Z} / p^{n}(r),
$$

for the natural maps $i: X_{1} \rightarrow X, j: X_{K} \rightarrow X$. We will explain how this map is defined (see [11], [19] for details). We say that a morphism $\mathcal{Z} \rightarrow \mathcal{Y}$ of $p$-adic formal schemes over $\operatorname{Spf}(W(k))$ is syntomic if every $Z_{n} \rightarrow Y_{n}$ is syntomic. For a formal scheme $\mathcal{Z}$ the syntomic-étale site $\mathcal{Z}_{\text {sét }}$ is defined by taking as objects morphisms $\mathcal{Y} \rightarrow \mathcal{Z}$ that are syntomic, quasi-finite and have étale generic fiber in the sense of rigid geometry. For a scheme $Z$, we also have the syntomic-étale site $Z_{\text {sét. }}$. Here the objects are morphisms $U \rightarrow Z$ that are syntomic, quasi-finite, and with $U_{K}$ étale over $Z_{K}$. Let $\widehat{X}$ be the $p$-adic completion of $X$. We have the following commutative diagram of topoi

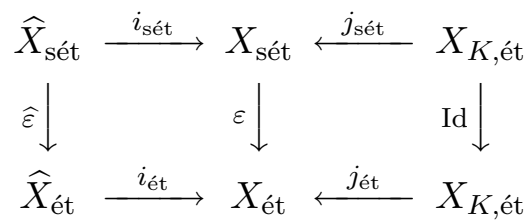

Abusively, let $\mathcal{S}_{n}(r)$ denote also the direct image of $\mathcal{S}_{n}(r)$ under the canonical morhpism $i: X_{n, \text { syn }} \rightarrow \widehat{X}_{\text {sét }}$. Since $i$ is exact [11, III.4.1], we have $R \widehat{\varepsilon}_{*} \mathcal{S}_{n}(r)=$ $R \varepsilon_{*} \mathcal{S}_{n}(r)$. By [11, III.5], there is a canonical homomorphism

$$
\alpha: \mathcal{S}_{n}(r) \rightarrow i_{\text {sét }}^{*} j_{\text {sét } *} \mathbf{Z} / p^{n}(r) .
$$

Let us recall its definition. Let $\operatorname{Spf}(\mathcal{B})$ be syntomic-étale, quasi-finite over $\widehat{X}$. Then $[11,4.3] \mathcal{B}$ is a completion of an algebra $B$ that is syntomic-étale, quasi-finite over $X$. The inverse image functor $i_{\text {sét }}^{*} \mathcal{F}$ is defined by sheafifying the presheaf $\mathcal{B} \mapsto \mathcal{F}\left(B^{h}\right)$, where $B^{h}$ is the henselization of $B$ with respect to $p$. It follows that to define the map $\alpha$ it suffices to construct functorial maps

$$
\alpha_{\mathcal{B}}: S_{n}(r)(\mathcal{B}) \rightarrow \mathbf{Z} / p^{n}(r)\left(B^{h}[1 / p]\right) .
$$

We may assume that $B$ is a domain (cf., $[11,5.1])$ and that $\operatorname{Spec}(B / p B)$ is connected. Then the fundamental exact sequence (2.2) yields an exact sequence 
of continuous $\operatorname{Gal}\left(\overline{B^{h}} / B^{h}\right)$-modules

$$
0 \rightarrow \mathbf{Z} / p^{n}(r)\left(\overline{B^{h}}\right) \rightarrow F^{r} B_{\mathrm{cr}, n}^{+}\left(\overline{B^{h}}\right) \stackrel{p^{-r} \phi-1}{\longrightarrow} B_{\mathrm{cr}, n}^{+}\left(\overline{B^{h}}\right) \rightarrow 0 .
$$

Let $\widetilde{B^{h}}$ denote the image of the map $\theta: B_{\mathrm{cr}}^{+}\left(\overline{B^{h}}\right) \rightarrow \widehat{\overline{B^{h}}}$. We have a natural isomorphism

$$
H_{\mathrm{cr}}^{0}\left(\widetilde{B}_{n}^{h} / W_{n}(k), J_{n}^{[r]}\right) \stackrel{\sim}{\rightarrow} F^{r} B_{\mathrm{cr}, n}^{+}\left(\overline{B^{h}}\right) .
$$

There is also a morphism $B \rightarrow \widetilde{B^{h}}$ [31, Lemma 1.4.1], hence a natural map

$$
H_{\mathrm{cr}}^{0}\left(B_{n} / W_{n}(k), J_{n}^{[r]}\right) \rightarrow H_{\mathrm{cr}}^{0}\left(\widetilde{B_{n}^{h}} / W_{n}(k), J_{n}^{[r]}\right) .
$$

Composing these two morphisms we get a map $H_{\mathrm{cr}}^{0}\left(B_{n} / W_{n}(k), J_{n}^{[r]}\right) \rightarrow F^{r} B_{\mathrm{cr}, n}^{+}\left(\overline{B^{h}}\right)$, that is clearly compatible with Frobenius. This map yields our morphism $\alpha_{\mathcal{B}}$ via maping

$$
S_{n}(r)(B)=\operatorname{Ker}\left(H_{\mathrm{cr}}^{0}\left(B_{n} / W_{n}(k), J_{n}^{[r]}\right) \stackrel{p^{-r} \phi-1}{\longrightarrow} H_{\mathrm{cr}}^{0}\left(B_{n} / W_{n}(k)\right)\right)
$$

into $H^{0}\left(\operatorname{Gal}\left(\overline{B^{h}} / B^{h}\right), \mathbf{Z} / p^{n}(r)\left(\overline{B^{h}}\right)\right)=\mathbf{Z} / p^{n}(r)\left(B^{h}[1 / p]\right)$ using the fundamental exact sequence above.

Having defined the map $\alpha$, we can apply $R \widehat{\varepsilon}_{*}$ to the induced map $\mathcal{S}_{n}(r) \rightarrow$ $i_{\text {sét }}^{*} R j_{\text {sét } *} \mathbf{Z} / p^{n}(r)$ and get

$$
R \varepsilon_{*} \mathcal{S}_{n}(r)=R \widehat{\varepsilon}_{*} \mathcal{S}_{n}(r) \rightarrow R \widehat{\varepsilon}_{*} i_{\text {sét }}^{*} R j_{\text {sét } *} \mathbf{Z} / p^{n}(r)=i_{\text {ét }}^{*} R \varepsilon_{*} R j_{\text {sét } *} \mathbf{Z} / p^{n}(r)=i^{*} R j_{*} \mathbf{Z} / p^{n}(r) .
$$

The second equality was proved in $[19,2.5]$. Since $R^{q} \varepsilon_{*} \mathcal{S}_{n}(r)=0$ for $q>r$, the map $\alpha_{r}$ factors through $\tau_{\leq r} i^{*} R j_{*} \mathbf{Z} / p^{n}(r)$. One checks that $\alpha_{r}$ is compatible with products.

Theorem 4.2. ([16, I.4.3], [21]) Taking the limit over finite extensions of $V$ in $\bar{V}$, the period map $\alpha_{r}$ induces a quasi-isomorphism

$$
\alpha_{r}^{K}: \mathcal{S}_{n}(r)_{X_{\bar{V}, n}} \stackrel{\sim}{\rightarrow} \tau_{\leq r} \bar{i}^{*} R \bar{j}_{*} \mathbf{Z} / p^{n}(r),
$$

where $\bar{i}: X_{\bar{V}, n} \hookrightarrow X_{\bar{V}}, \bar{j}: X_{\bar{K}} \hookrightarrow X_{\bar{V}}$ are the canonical maps.

For $X$ smooth and proper over $V$ and $r \geq i$, the integral crystalline period map is defined as

$$
\alpha_{i, r}^{K}: H^{i}\left(X_{\bar{V}}, \mathcal{S}_{n}(r)\right) \stackrel{\alpha_{r}^{K}}{\sim} H^{i}\left(X_{\bar{K}}, \mathbf{Z} / p^{n}(r)\right) .
$$

It is an isomorphism 
Similarly, for any $r \geq 0$, we get a natural map

$$
\alpha_{r}: \mathcal{S}_{n}(r) \rightarrow \tau_{\leq r} i^{*} R j_{*} \mathbf{Z} / p^{n}(r)^{\prime},
$$

where $\mathbf{Z} / p^{n}(r)^{\prime}=\left(p^{a} a !\right)^{-1} \mathbf{Z} / p^{n}(r)$ for $r=(p-1) a+b, a, b \in \mathbf{Z}, 0 \leq b<p-1$ [11, III.5]. Composing with the map $\mathcal{S}_{n}^{\prime}(r) \rightarrow \mathcal{S}_{n}(r)$ we get a natural, compatible with products, morphism

$$
\alpha_{r}: \mathcal{S}_{n}^{\prime}(r) \rightarrow \tau_{\leq r} i^{*} R j_{*} \mathbf{Z} / p^{n}(r)^{\prime} .
$$

Theorem 4.3. ([31, 3.3.4]) For any $0 \leq i \leq r$, the kernel and cokernel of the period map

$$
\alpha_{r}: \mathcal{H}^{i}\left(\mathcal{S}_{n}^{\prime}(r)_{X_{\bar{V}, n}}\right) \rightarrow \bar{i}^{*} R^{i} \bar{j}_{*} \mathbf{Z} / p^{n}(r)^{\prime},
$$

is annihilated by $p^{N}$ for an integer $N$ which depends only on $p, r$, and $i$.

For $X$ smooth and proper over $V$ and $r \geq i$, the rational crystalline period map is defined as

$$
\alpha_{i, r}^{T}: H^{i}\left(X_{\bar{V}}, \mathcal{S}_{\mathbf{Q}_{p}}^{\prime}(r)\right) \stackrel{\alpha_{r}}{\longrightarrow} H^{i}\left(X_{\bar{K}}, \mathbf{Q}_{p}(r)\right) \stackrel{p^{-r}}{\longrightarrow} H^{i}\left(X_{\bar{K}}, \mathbf{Q}_{p}(r)\right),
$$

where $H^{i}\left(X_{\bar{V}}, \mathcal{S}_{\mathbf{Q}_{p}}^{\prime}(r)\right)=\mathbf{Q}_{p} \otimes \operatorname{proj} \lim _{n} H^{i}\left(X_{\bar{V}}, \mathcal{S}_{n}^{\prime}(r)\right)$. It yields an isomorphism

$$
\alpha_{i, r}^{T}: H^{i}\left(X_{\bar{V}}, \mathcal{S}_{\mathbf{Q}_{p}}^{\prime}(r)\right) \stackrel{\sim}{\rightarrow} H^{i}\left(X_{\bar{K}}, \mathbf{Q}_{p}(r)\right) .
$$

The above definitions extend to $\log$-schemes [4], [30]. We have sheaves $\mathcal{O}_{n}^{\text {cr }}$, $\mathcal{J}_{n}^{[r]}, r \geq 0$, on the small log-syntomic site of $\operatorname{Spec}(W(k))$ defined by

$\mathcal{O}_{n}^{\mathrm{cr}}\left(X^{\times}\right)=H_{\mathrm{cr}}^{0}\left(X_{n}^{\times} / W_{n}(k), \mathcal{O}_{X_{n}^{\times} / W_{n}(k)}\right), \mathcal{J}_{n}^{[r]}\left(X^{\times}\right)=H_{\mathrm{cr}}^{0}\left(X_{n}^{\times} / W_{n}(k), \mathcal{J}_{X_{n}^{\times} / W_{n}(k)}^{[r]}\right)$, and a functorial isomorphisms

$$
H_{\mathrm{syn}}^{*}\left(X^{\times}, \mathcal{J}_{n}^{[r]}\right) \simeq H_{\mathrm{cr}}^{*}\left(X_{n}^{\times} / W_{n}(k), \mathcal{J}_{X_{n}^{\times} / W_{n}(k)}^{[r]}\right) .
$$

We get the induced sheaves $\mathcal{J}_{n}^{[r]}$, the log-syntomic complexes

$$
\mathcal{S}_{n}(r):=\operatorname{Ker}\left(\mathcal{J}_{n}^{<r>} \stackrel{\phi_{r}-1}{\longrightarrow} \mathcal{O}_{n}^{\mathrm{cr}}\right), \quad \mathcal{S}_{n}^{\prime}(r):=\operatorname{Ker}\left(\mathcal{J}_{n}^{[r]} \stackrel{\phi-p^{r}}{\longrightarrow} \mathcal{O}_{n}^{\mathrm{cr}}\right),
$$

and, for $r \geq 0$, the exact sequence $[3,3.1 .4]$

$$
0 \longrightarrow \mathcal{S}_{n}(r) \longrightarrow \mathcal{J}_{n}^{<r>} \stackrel{\phi_{r}-1}{\longrightarrow} \mathcal{O}_{n}^{\text {cr }} \longrightarrow 0 .
$$


Let $X^{\times}$be a log-smooth vertical scheme over $V^{\times}$. Then the above construction of the period morphism carries over almost verbatim to yield natural morphisms on the étale site of $X^{\times}[30]$

$$
\begin{aligned}
& \alpha_{r}: \mathcal{S}_{n}(r) \rightarrow \tau_{\leq r} i^{*} R j_{*} \mathbf{Z} / p^{n}(r), \quad 0 \leq r \leq p-2 \\
& \alpha_{r}: \mathcal{S}_{n}^{\prime}(r) \rightarrow \tau_{\leq r} i^{*} R j_{*} \mathbf{Z} / p^{n}(r)^{\prime}, \quad r \geq 0 .
\end{aligned}
$$

We have the following comparison theorem proved by Tsuji [32, Theorem 5.1], $[31,3.3 .4]$. Notice that here we work on $X^{\times}$not on $X_{\bar{V}}^{\times}$as we did in the crystalline case.

Theorem 4.4. (1) Let $X^{\times}$be a fine and saturated, vertical, log-smooth scheme over $V^{\times}$. Then for $0 \leq r \leq p-2$, the period map $\alpha_{r}$ induces a quasiisomorphism

$$
\alpha_{r}: \mathcal{S}_{n}(r)_{X_{n}^{\times}} \stackrel{\sim}{\rightarrow} \tau_{\leq r} i^{*} R j_{*} \mathbf{Z} / p^{n}(r)_{X_{K}^{\times}}
$$

(2) Let $X^{\times}$be vertical semistable over $V^{\times}$or a finite base change of such, then for any $0 \leq i \leq r$, the kernel and cokernel of the period map

$$
\alpha_{r}: \mathcal{H}^{i}\left(\mathcal{S}_{n}^{\prime}(r)_{X_{n}^{\times}}\right) \rightarrow i^{*} R^{i} j_{*} \mathbf{Z} / p^{n}(r)_{X_{K}^{\times}}^{\prime},
$$

is annihilated by $p^{N}$ for an integer $N$ which depends only on $p, r$, and $i$.

For $X^{\times}$fine and saturated, vertical, $\log$-smooth and proper over $V^{\times}$and $r \geq i$, the rational semistable period map is defined as

$$
\alpha_{i, r}^{T}: H^{i}\left(X^{\times}, \mathcal{S}_{\mathbf{Q}_{p}}^{\prime}(r)\right) \stackrel{\alpha_{r}}{\longrightarrow} H^{i}\left(X_{K}^{\times}, \mathbf{Q}_{p}(r)\right) \stackrel{p^{-r}}{\longrightarrow} H^{i}\left(X_{K}^{\times}, \mathbf{Q}_{p}(r)\right),
$$

where $H^{i}\left(X^{\times}, \mathcal{S}_{\mathbf{Q}_{p}}^{\prime}(r)\right)=\mathbf{Q}_{p} \otimes \operatorname{proj} \lim _{n} H^{i}\left(X^{\times}, \mathcal{S}_{n}^{\prime}(r)\right)$. For $X^{\times}$vertical and semistable it yields an isomorphism

$$
\alpha_{i, r}^{T}: H^{i}\left(X^{\times}, \mathcal{S}_{\mathbf{Q}_{p}}^{\prime}(r)\right) \stackrel{\sim}{\rightarrow} H^{i}\left(X_{K}^{\times}, \mathbf{Q}_{p}(r)\right) .
$$

Lemma 4.5. Let $X^{\times}$over $V^{\times}$be a fine and saturated, vertical log-smooth scheme. Then, for $r \geq i$, we have an isomorphism

$$
\alpha_{i, r}^{T}: H^{i}\left(X_{\bar{V}}^{\times}, \mathcal{S}_{\mathbf{Q}_{p}}^{\prime}(r)\right) \stackrel{\sim}{\rightarrow} H^{i}\left(X_{\bar{K}}, \mathbf{Q}_{p}(r)\right) .
$$

Proof. By [27, Theorem 2.9] there exists a ramified extension $V_{1}$ of $V$ such that the base change $X_{V_{1}^{\times}}^{\times}$has a semistable model. That is, there exists a log-blow-up 
$\pi: Y^{\times} \rightarrow X_{V_{1}^{\times}}^{\times}$such that $Y^{\times}$is a semistable scheme (with no multiplicities in the special fiber). We have the following commutative diagram

$$
\begin{gathered}
H^{i}\left(Y_{\bar{V}}^{\times}, \mathcal{S}_{\mathbf{Q}_{p}}^{\prime}(r)\right) \stackrel{\alpha_{i, r}^{T}}{\sim} H^{i}\left(X_{\bar{K}}, \mathbf{Q}_{p}(r)\right) \\
\pi^{*} \uparrow \text { ? } \\
H^{i}\left(X_{\bar{V}}^{\times}, \mathcal{S}_{\mathbf{Q}_{p}}^{\prime}(r)\right) \stackrel{\alpha_{i, r}^{T}}{\longrightarrow} H^{i}\left(X_{\bar{K}}, \mathbf{Q}_{p}(r)\right) .
\end{gathered}
$$

Since log-blow-ups do not change the syntomic cohomology [25, Proposition 2.3] and the top horizontal map is an isomorphism, our lemma follows.

Corollary 4.6. Let $X^{\times}$be a proper, log-smooth, vertical, fine and saturated $V^{\times}$scheme with Cartier type reduction. The natural $B_{\mathrm{st}}$-linear Galois equivariant period morphism

$$
\alpha_{i, r}^{T}: H^{i}\left(X_{\bar{K}}, \mathbf{Q}_{p}(r)\right) \otimes_{\mathbf{Q}_{p}} B_{\mathrm{st}} \rightarrow H_{\mathrm{cr}}^{i}\left(X_{0}^{\times} / W(k)^{0}\right) \otimes_{W(k)} B_{\mathrm{st}}\{-r\}
$$

is an isomorphism. It preserves the Frobenius and the monodromy operators, and, after extension to $B_{d R}$, induces an isomorphism of filtrations.

Proof. Having the above lemma, the standard proof ([31, Theorem 4.10.2]) goes through.

\subsection{Compatibility with higher Chern classes.}

Theorem 4.7. Let $X$ be smooth and proper over $V=W(k)$ and let $0 \leq r \leq p-2$. For $2 r \geq i+2$ we have the following commutative diagram

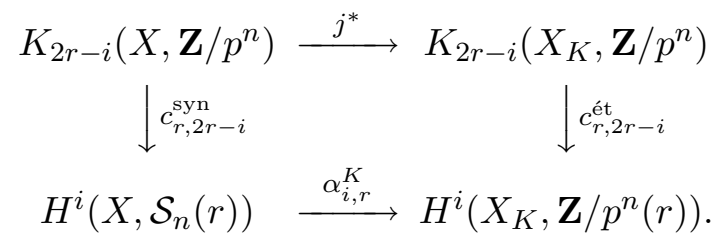

Similarly for integral $K$-theory and $2 r \geq i$.

Proof. First, we need a definition of Chern classes

$$
\begin{aligned}
& c_{i, j}: K_{j}\left(X, \mathbf{Z} / p^{n}\right) \rightarrow H^{2 i-j}\left(X, i_{*} i^{*} R j_{*} \mathbf{Z} / p^{n}(i)\right), \\
& c_{i, j}: K_{j}(X) \rightarrow H^{2 i-j}\left(X, i_{*} i^{*} R j_{*} \mathbf{Z} / p^{n}(i)\right) .
\end{aligned}
$$


Set $c_{0}: \mathbf{Z} / p^{n} \rightarrow i_{*} i^{*} R j_{*} \mathbf{Z} / p^{n}$ equal to the map induces from the localization map $\mathbf{Z} / p^{n} \rightarrow j_{*} \mathbf{Z} / p^{n}$. Set $c_{1}: \mathcal{O}_{X}^{*}[-1] \rightarrow i_{*} i^{*} R j_{*} \mathbf{Z} / p^{n}(1)$ equal to the composition

$$
\mathcal{O}_{X}^{*}[-1] \rightarrow i_{*} i^{*} R j_{*} \mathcal{O}_{X_{K}}^{*}[-1] \rightarrow i_{*} i^{*} R j_{*} \mathbf{Z} / p^{n}(1)
$$

where the last map is induced from the class $c_{1}: \mathcal{O}_{X_{K}}^{*}[-1] \rightarrow \mathbf{Z} / p^{n}(1)$ given by Kummer theory.

Proposition 4.8. Let $m>0, \xi=c_{1}(\mathcal{O}(1)) \in H^{2}\left(\mathbf{P}_{X}^{m}, i_{*} i^{*} R j_{*} \mathbf{Z} / p^{n}(1)\right)$. Let $\pi_{X}$ be the projection $\mathbf{P}_{X}^{m} \rightarrow X$, then the natural map

$$
\oplus_{i} \xi^{i} \cup \pi_{X}^{*}: \bigoplus_{i=0}^{m} i_{*} i^{*} R j_{*} \mathbf{Z} / p^{n}(r-i)_{X_{K}}[-2 i] \rightarrow R \pi_{X *} i_{*} i^{*} R j_{*} \mathbf{Z} / p^{n}(r)_{\mathbf{P}_{X_{K}}^{m}}
$$

is a quasi-isomorphism for all $r$.

Proof. Notice that by construction $\xi$ is the image of the étale class $\xi_{1}=c_{1}(\mathcal{O}(1)) \in$ $H^{2}\left(\mathbf{P}_{X_{K}}^{m}, \mathbf{Z} / p^{n}(1)\right)$ under the natural map $H^{2}\left(\mathbf{P}_{X_{K}}^{m}, \mathbf{Z} / p^{n}(1)\right) \rightarrow H^{2}\left(\mathbf{P}_{X}^{m}, i_{*} i^{*} R j_{*}\right.$ $\mathbf{Z} / p^{n}(1)$ ). Since $\pi_{X *} i_{*}=i_{*} \pi_{X_{k^{*}}}$ and $R \pi_{X_{k^{*}}} i^{*} \simeq i^{*} R \pi_{X *}$ (proper base change theorem), we easily reduce to the statement that for every $l$, the morphism

$$
\oplus_{i} \xi_{1}^{i} \cup \pi_{X}^{*}: \bigoplus_{i=0}^{m} H^{l-2 i}\left(X_{K}, \mathbf{Z} / p^{n}(r-i)\right) \rightarrow H^{l}\left(\mathbf{P}_{X_{K}}^{m}, \mathbf{Z} / p^{n}(r)\right)
$$

is an isomorphism. But this is just the projective space theorem in étale cohomology.

Like in $[13,2.2]$ we can now construct universal classes $C_{i} \in H^{2 i}(B . G L / V$, $\left.i_{*} i^{*} R j_{*} \mathbf{Z} / p^{n}(i)\right)$ that are compatible with the étale universal classes $C_{i}^{\text {ét }} \in H^{2 i}$ $\left(B . G L / K, \mathbf{Z} / p^{n}(i)\right)$. We claim that they are also compatible with the syntomic universal classes $C_{i}^{\mathrm{syn}} \in H^{2 i}\left(B . G L / V, S_{n}(i)\right)$, for $i \leq p-2$ (via the map $\alpha_{r}$ ). Indeed, the classes $C_{i}^{\mathrm{syn}} \in H^{2 i}\left(B . G L / V, S_{n}(i)\right)$, for $i \leq p-2$, are pullbacks of classes $C_{i, m}^{\mathrm{syn}} \in H^{2 i}\left(B . G L_{m} / V, S_{n}(i)\right)$, for $i \leq m \leq p-2$, and those can be defined using the projective space theorem in syntomic cohomology quoted below and Chern classes

$$
c_{0}^{\mathrm{syn}}: \mathbf{Z} / p^{n} \rightarrow \mathcal{S}_{n}(0)=\left(\mathcal{O}_{n}^{\mathrm{cr}}\right)^{\phi=1}, \quad c_{1}^{\mathrm{syn}}: \mathcal{O}_{X}^{*}[-1] \rightarrow i_{*} \mathcal{O}_{X_{n+1}}^{*}[-1] \rightarrow i_{*} \mathcal{S}_{n}(1) .
$$

Here, $c_{0}^{\text {syn }}$ is the canonical map and $c_{1}^{\text {syn }}$ is the symbol map defined in [16, I.3]. 
Proposition 4.9. ([14, I.4.3]) Let $p-1 \geq r \geq m>0$, $\xi_{\mathrm{syn}}=c_{1}(\mathcal{O}(1)) \in$ $H^{2}\left(\mathbf{P}_{X}^{m}, \mathcal{S}_{n}(1)\right)$. Let $\pi_{X}$ be the projection $\mathbf{P}_{X}^{m} \rightarrow X$, then we have a quasiisomorphism

$$
\oplus_{i} \xi_{\mathrm{syn}}^{i} \cup \pi_{X}^{*}: \bigoplus_{i=0}^{m} \mathcal{S}_{n}(r-i)_{X}[-2 i] \rightarrow R \pi_{X *} \mathcal{S}_{n}(r)_{\mathbf{P}_{X}^{m}}
$$

Since the morphism $\alpha_{r}$ is compatible with products and Chern classes $c_{0}$, $c_{1}$ (see [31, 3.2.4]), comparing the two projective space theorems yields that $\alpha_{r}\left(C_{i, m}^{\mathrm{syn}}\right)=C_{i, m}$, for $i \leq m \leq p-2$. By construction of higher Chern classes this suffices to prove our theorem.

Theorem 4.10. Let $X$ be smooth and proper over $V$. For $2 r \geq i+2$, we have the following commutative diagram

$$
\begin{array}{rrr}
K_{2 r-i}\left(X, \mathbf{Z} / p^{n}\right) & \stackrel{j^{*}}{\longrightarrow} & K_{2 r-i}\left(X_{K}, \mathbf{Z} / p^{n}\right) \\
\downarrow c_{r, 2 r-i}^{\text {syn }} & & \downarrow p^{r} c_{r, 2 r-i}^{\text {et }} \\
H^{i}\left(X, \mathcal{S}_{n}^{\prime}(r)\right) & \stackrel{\alpha_{i, r}^{T}}{\longrightarrow} H^{i}\left(X_{K}, \mathbf{Z} / p^{n}(r)^{\prime}\right) .
\end{array}
$$

Similarly for integral $K$-theory and $2 r \geq i$. Here the étale Chern classes are defined as the composition

$$
c_{r, 2 r-i}^{\text {ét }}: \quad K_{2 r-i}\left(X_{K}, \mathbf{Z} / p^{n}\right) \stackrel{c_{r, 2 r-i}^{\text {ét }}}{\longrightarrow} H^{i}\left(X_{K}, \mathbf{Z} / p^{n}(r)\right) \rightarrow H^{i}\left(X_{K}, \mathbf{Z} / p^{n}(r)^{\prime}\right),
$$

where the last map is induced by the map $1 \mapsto p^{a} a$ !, for $r=a(p-1)+b, 0 \leq b<$ $p-1$.

Proof. By Lemma 2.7, it is enough to show that the following diagram commutes

$$
\begin{array}{rcc}
K_{2 r-i}\left(X, \mathbf{Z} / p^{n}\right) & \stackrel{j^{*}}{\longrightarrow} & K_{2 r-i}\left(X_{K}, \mathbf{Z} / p^{n}\right) \\
\downarrow c_{r, 2 r-i}^{\mathrm{syn}} & & \downarrow c_{r, 2 r-i}^{\text {et }} \\
H^{i}\left(X, \mathcal{S}_{n}(r)\right) & \stackrel{\alpha_{r}}{\longrightarrow} H^{i}\left(X_{K}, \mathbf{Z} / p^{n}(r)^{\prime}\right) .
\end{array}
$$

Note that here $i$ can be arbitrarily large. We have the following special case of the projective space theorem.

Proposition 4.11. Let $m>0, X=B . G L_{m+1} / W(k)$. Take $\xi_{\text {syn }}=c_{1}^{\text {syn }}(\mathcal{O}(1)) \in$ $H^{2}\left(\mathbf{P}_{X}^{m}, \mathcal{S}_{n}(1)\right)$. Let $\pi_{X}$ be the projection $\mathbf{P}_{X}^{m} \rightarrow X$, then the natural map

$$
\oplus_{i} \xi_{\mathrm{syn}}^{i} \cup \pi_{X}^{*}: \bigoplus_{i=0}^{m} H^{l-2 i}\left(X, \mathcal{S}_{n}(r-i)\right) \rightarrow H^{l}\left(\mathbf{P}_{X}^{m}, \mathcal{S}_{n}(r)\right)
$$


is an isomorphism for all $r \geq m$.

Proof. This follows immediately from the projective space theorems in the de Rham cohomology:

$$
\begin{aligned}
& \oplus_{i} \xi_{d R}^{i} \cup \pi_{X}^{*}: \bigoplus_{i=0}^{m} F^{r-i} H_{d R}^{l-2 i}\left(X_{n} / W_{n}(k)\right) \stackrel{\sim}{\rightarrow} F^{r} H_{d R}^{l}\left(\mathbf{P}_{X_{n}}^{m} / W_{n}(k)\right), \\
& \oplus_{i} \xi_{d R}^{i} \cup \pi_{X}^{*}: \bigoplus_{i=0}^{m} H_{d R}^{l-2 i}\left(X_{n} / W_{n}(k)\right) \stackrel{\sim}{\rightarrow} H_{d R}^{l}\left(\mathbf{P}_{X_{n}}^{m} / W_{n}(k)\right) .
\end{aligned}
$$

Having that, the proof of Theorem 4.7 goes through.

The following corollary follows immediately.

Corollary 4.12. Let $X$ be smooth and proper over $V$. For $2 r \geq i+2$ we have the following commutative diagram

$$
\begin{aligned}
& \mathbf{Q}_{p} \otimes \operatorname{proj} \lim _{n} K_{2 r-i}\left(X, \mathbf{Z} / p^{n}\right) \stackrel{j^{*}}{\longrightarrow} \mathbf{Q}_{p} \otimes \operatorname{proj} \lim _{n} K_{2 r-i}\left(X, \mathbf{Z} / p^{n}\right) \\
& \downarrow c_{r, 2 r-i}^{\mathrm{syn}} \quad \downarrow c_{r, 2 r-i}^{\mathrm{et}} \\
& H^{i}\left(X, \mathcal{S}_{\mathbf{Q}_{p}}^{\prime}(r)\right) \quad \stackrel{\alpha_{i, r}^{T}}{\longrightarrow} \quad H^{i}\left(X, \mathbf{Q}_{p}(r)\right) .
\end{aligned}
$$

Similarly for integral $K$-theory and $2 r \geq i$.

Theorem 4.13. Let $X^{\times}$be fine and saturated, log-smooth, vertical and proper over $V^{\times}$. For $2 r \geq i+2$ we have the following commutative diagram

$$
\begin{array}{rcc}
K_{2 r-i}\left(X, \mathbf{Z} / p^{n}\right) & \stackrel{j^{*}}{\longrightarrow} & K_{2 r-i}\left(X_{K}, \mathbf{Z} / p^{n}\right) \\
\downarrow c_{r, 2 r-i}^{\mathrm{syn}} & & \downarrow c_{r, 2 r-i}^{\text {ét }} \\
H^{i}\left(X^{\times}, \mathcal{S}_{n}(r)\right) \stackrel{\alpha_{i, r}^{T}}{\longrightarrow} & H^{i}\left(X_{K}, \mathbf{Z} / p^{n}(r)^{\prime}\right) .
\end{array}
$$

Similarly for integral $K$-theory and $2 r \geq i$. Here the Chern class $c_{r, 2 r-i}^{\mathrm{syn}}$ is the composition of the classical syntomic Chern class $K_{2 r-i}\left(X, \mathbf{Z} / p^{n}\right) \stackrel{c_{r, 2 r-i}^{\mathrm{syn}}}{\longrightarrow} H^{i}\left(X, \mathcal{S}_{n}(r)\right)$ with the natural map $H^{i}\left(X, \mathcal{S}_{n}(r)\right) \rightarrow H^{i}\left(X^{\times}, \mathcal{S}_{n}(r)\right)$. 
Proof. Arguing as in the proof of Theorem 4.7, we get Chern classes into the étale cohomology of $i_{*} i^{*} R j_{*} \mathbf{Z} / p^{n}(i)$ compatible with the étale Chern classes on the generic fiber $X_{K}$. It suffices now to show that the map

$$
\alpha_{r}^{\prime}: \mathcal{S}_{n}(r)_{X} \rightarrow \mathcal{S}_{n}(r)_{X^{\times}} \stackrel{\alpha_{r}}{\longrightarrow} i_{*} i^{*} R j_{*} \mathbf{Z} / p^{n}(i)_{X_{K}}
$$

is compatible with Chern classes from $K_{*}\left(X, \mathbf{Z} / p^{n}\right)$. Using Proposition 4.11 (projective space theorem for the classifying space $B . G L_{m} / W(k)$ ), we reduce to showing that the classical syntomic Chern classes $c_{0}^{\mathrm{syn}}, c_{1}^{\mathrm{syn}}$ commute with Chern classes $c_{0}, c_{1}$ (notation as in the proof of Theorem 4.7) via the morphism $\alpha_{r}^{\prime}$. But this was checked by Tsuji in [31, 3.2.4].

Corollary 4.14. Let $X^{\times}$be fine and saturated, log-smooth, vertical and proper over $V^{\times}$. For $2 r \geq i+2$ we have the following commutative diagram

$$
\begin{array}{ccc}
\mathbf{Q}_{p} \otimes \operatorname{proj} \lim _{n} K_{2 r-i}\left(X \frac{\times}{V}, \mathbf{Z} / p^{n}\right) & \stackrel{j^{*}}{\longrightarrow} & \mathbf{Q}_{p} \otimes \operatorname{proj} \lim _{n} K_{2 r-i}\left(X_{\bar{K}}, \mathbf{Z} / p^{n}\right) \\
\downarrow_{r, 2 r-i}^{\text {syn }} & & \downarrow_{r, 2 r-i}^{\text {et }} \\
H^{i}\left(X_{\bar{V}}^{\times}, \mathcal{S}_{\mathbf{Q}_{p}}^{\prime}(r)\right) & \stackrel{\alpha_{i, r}^{T}}{\longrightarrow} & H^{i}\left(X_{\bar{K}}, \mathbf{Q}_{p}(r)\right) .
\end{array}
$$

Similarly for integral $K$-theory and $2 r \geq i$.

Proof. Let $Y^{\times}$be a $\log$-smooth, vertical, proper scheme over a finite extension $V_{1}$ of $V$ with fraction field $K_{1}$. Then, by the above theorem and Lemma 2.7, we get a commutative diagram

$$
\begin{array}{rrr}
K_{2 r-i}\left(Y, \mathbf{Z} / p^{n}\right) & j^{*} & K_{2 r-i}\left(Y_{K_{1}}, \mathbf{Z} / p^{n}\right) \\
\downarrow c_{r, 2 r-i}^{\mathrm{syn}} & & \\
H^{i}\left(Y^{\times}, \mathcal{S}_{n}^{\prime}(r)\right) & \stackrel{\alpha_{i, r}^{T}}{\longrightarrow} H_{r, 2 r-i}{ }^{i}\left(Y_{K_{1}}, \mathbf{Z} / p^{n}(r)^{\prime}\right) .
\end{array}
$$

Our corollary follows.

\section{FALtings PERIOD MORPHISMS}

Faltings construction of the period morphisms uses an auxiliary topos, topos of "sheaves of local systems" [6, III], [7, 3]. We will now describe it. For a scheme $X$, let $X_{\text {Fét }}$ denote the topos defined by the site of finite étale morphisms $U \rightarrow X$ with coverings given by surjective maps. For a connected $X$ and a choice of a geometric point $\bar{x} \rightarrow X, X_{\text {Fét }}$ is equivalent to the topos of sets $F_{\bar{x}}$ with 
a continuous action of the fundamental group $\pi_{1}(X, \bar{x})$. In particular, for an abelian sheaf $F$, the étale cohomology $H^{*}\left(X_{\text {Fét }}, F\right)$ is isomorphic to the group cohomology $H^{*}\left(\pi_{1}(X, \bar{x}), F_{\bar{x}}\right)$. Let $X$ be noetherian. Then $X_{\text {Fét }}$ is equivalent to the topos of étale sheaves that are inductive limits of locally constant sheaves. There is a map of topoi

$$
\pi: X_{\text {ét }} \rightarrow X_{\text {Fét }}
$$

with $\pi_{*} \mathcal{F}$ given by the restriction of $\mathcal{F}$ to finite étale schemes over $X$ and $\pi^{*}(L)=$ $L$ for an ind-locally constant sheaf $L$. Recall the following notion.

Definition 5.1. A noetherian scheme $X$ is a $K(\pi, 1)$-space if for every integer $n$ invertible on $X$ and any locally constant sheaf $L$ of $\mathbf{Z} / n$-modules, the natural map $L \rightarrow R \pi_{*} \pi^{*}(L)$ is an isomorphism.

Faltings [5, 2.1] proves the following generalization of a classical result on the existence of a base for the Zariski topology consisting of $K(\pi, 1)$-spaces.

Theorem 5.2. Let $X$ be a smooth $V$-scheme. Then any point $x \in X$ is contained in an open $U \subset X$ such that $U \times_{V} \bar{K}$ is a $K(\pi, 1)$-space.

It follows that for $X / V$ smooth, every point has a Zariski neighbourhood such that $U_{K}$ is a $K(\pi, 1)$-space.

Let $X$ be a noetherian $V$-scheme. Let $\widetilde{X}_{\text {ét }}$ be the following category. An object of $\widetilde{X}_{\text {ét }}$ is a collection $L=\left(\left(L_{U}\right),\left(r_{U_{1} U_{2}}\right)\right)$ of ind-locally constant étale sheaves $L_{U}$ on $U_{K}$, for every étale open $U$ of $X$ and, for every pair $U_{2} \rightarrow U_{1}$, a morphism $r_{U_{1} U_{2}}: L_{U_{1}} \mid\left(U_{2}\right)_{K} \rightarrow L_{U_{2}}$ such that $r_{U_{2} U_{3}} r_{U_{1} U_{2}}=r_{U_{1} U_{3}}$ and $r_{U U}=$ Id. One also requires that for every tranquated étale hypercovering $U_{1} \rightrightarrows U_{0} \rightarrow U, L_{U}$ is the maximal ind-locally constant subsheaf of $\operatorname{Ker}\left(j_{0 *} L_{U_{0}} \rightrightarrows j_{1 *} L_{U_{1}}\right)$, where $j_{i}:\left(U_{i}\right)_{K} \rightarrow U_{K}$. Morphism $f: L \rightarrow M$ in $\widetilde{X}$ is a collection of morphisms of ind-locally constant sheaves $f_{U}: L_{U} \rightarrow M_{U}$ compatible with the restrictions $r_{U_{1} U_{2}}$.

The category $\widetilde{X}_{\text {ét }}$ is a topos. We will sometimes denote by $\widetilde{X}_{\text {ét }}$ the equivalent topos, where all the $U^{\prime}$ 's are assumed to be affine. The underlying site of $\widetilde{X}_{\text {ét }}$ has for objects pairs $\left(U, U^{\prime}\right)$, where $U$ is an étale $X$-scheme and $U^{\prime} \rightarrow U_{K}$ is a finite étale morphism; morphisms are compatible pairs of maps and coverings are pairs of surjective maps. 
There is a canonical map

$$
\rho: X_{K, \text { ét }} \rightarrow \widetilde{X}_{\text {ét }}
$$

from the étale topos of $X_{K}$ to $\widetilde{X}_{\text {ét }}$. First, one equippes every étale and irreducible $U \rightarrow X$ with a geometric generic point, and every map $U_{1} \rightarrow U_{2}$ between two such étales with a path between the chosen points. Then the inverse image of $L$ by $\rho$ is the direct limit over all tranquated hypercoverings $U_{1} \rightarrow U_{0} \rightarrow X_{K}$ of $\operatorname{Ker}\left(j_{0 *} L_{U_{0}} \rightrightarrows j_{1 *} L_{U_{1}}\right)$; the direct image of $\mathcal{F}$ associates to $U$ the ind-locally constant subsheaf corresponding to the global sections of $\mathcal{F}$ on the universal covering of $U_{K}$. On the level of sites, this map is given by sending $\left(U, U^{\prime}\right)$ to $U^{\prime} \rightarrow X_{K}$. There is also a canonical map from $\widetilde{X}_{\text {ét }}$ to the étale topos of $X$

$$
\psi: \widetilde{X}_{\text {ét }} \rightarrow X_{\text {ét }}
$$

defined by the map of sites sending $U \rightarrow X$ to $\left(U, U_{K}\right)$. The pushforward $\psi_{*}(L)$ is the sheaf $U \mapsto L_{U}\left(U_{K}\right)$. Finally, we have a projection

$$
\widetilde{\pi}: \widetilde{X}_{\text {ét }} \rightarrow X_{K, \text { Fét }}
$$

induced by the map of sites sending $U \rightarrow X_{K}$ to $(X, U)$. The inverse image $\tilde{\pi}^{*} L$ associates to $j: U \rightarrow X$, the local system $j_{K}^{*} L$; the direct image $\widetilde{\pi}_{*} L$ is equal to $L_{X}$.

The above has a geometric version: topos $\widetilde{X}_{\bar{K} \text {,ét }}$ defined by pairs $\left(U, U^{\prime}\right)$ such that $U$ is étale over $X$ and $U^{\prime}$ is finite étale over $X_{\bar{K}}$, and the canonical maps of topoi:

$$
\rho: X_{\bar{K} \text {,ét }} \rightarrow \widetilde{X}_{\bar{K} \text {,ét }}, \quad \psi: \widetilde{X}_{\bar{K} \text {,ét }} \rightarrow X_{\text {ét }}, \quad \widetilde{\pi}: \widetilde{X}_{\bar{K} \text {,ét }} \rightarrow X_{\bar{K}, \text { Fét }} .
$$

Let $X$ be a smooth scheme over $V$. One checks [6, III] that, for an locally constant sheaf $L$ on $X_{K}$, there is an isomorphism

$$
H^{*}\left(\widetilde{X}_{\text {ét }}, \rho_{*} L\right) \simeq H^{*}\left(X_{K}, L\right) .
$$

Indeed, it suffices to show that $R^{k} \rho_{*} L=0$ for $k>0$. But $\left(R^{k} \rho_{*} L\right)_{U}=R^{k} \pi_{*} L_{U_{K}}$, where $\pi: U_{K \text {,ét }} \rightarrow U_{K, \text { Fét }}$. Hence it is trivial in the case $U$ is a $K(\pi, 1)$-space. Since such $U$ 's form a base for the topology of $X$, we are done. It follows that for an locally constant sheaf $L$ on $X_{\bar{K}}$, there is an isomorphism

$$
H^{*}\left(\widetilde{X}_{\bar{K}, \text { ét }}, \rho_{*} L\right) \simeq H^{*}\left(X_{\bar{K}}, L\right) .
$$

Define sheaves $\overline{\mathcal{O}}$ and $B_{\mathrm{cr}, n}^{+}$on the topos $\widetilde{X}_{\text {ét }}$ by associating to étale maps $\operatorname{Spec}(R) \rightarrow X$, the locally constant sheaves on $\operatorname{Spec}\left(R_{K}\right)$ defined by the Galois 
modules $\bar{R}$ and $B_{\mathrm{cr}, n}^{+}(\bar{R})$. Denote by $B_{\mathrm{cr}, n}^{+, 0}$ the subsheaf of $B_{\mathrm{cr}, n}^{+}$of sections mapping to $R_{n}$ via the map $\theta: B_{\mathrm{cr}, n}^{+}(\bar{R}) \rightarrow \bar{R}_{n}$. The last map is a surjection for $R$ small, i.e., admitting an étale map from $V\left[T_{1}^{ \pm}, \ldots, T_{k}^{ \pm}\right]$(since Frobenius is surjective on $\bar{R} / p)$, hence, locally on $\widetilde{X}_{\text {ét }}, B_{\mathrm{cr}, n}^{+, 0}$ is a PD-thickening of $R_{n}$ over $W_{n}(k)$. There is a cohomological, base-point free definition of these sheaves [20, 2.3]. For an element $\left(U=\operatorname{Spec}(H), U^{\prime}\right)$ of the site of $\widetilde{X}_{\text {ét }}$, denote by $H^{\prime}$ the normalization of $H$ in $U^{\prime}$. The sheaf $\overline{\mathcal{O}}$ is defined be sending $\left(U, U^{\prime}\right)$ to $H^{\prime}$. The sheaf $B_{\mathrm{cr}, n}^{+}$is associated to the presheaf sending $\left(U, U^{\prime}\right)$ to the degree zero crystalline cohomology of $H_{n}^{\prime}$ over $W_{n}(k)$ and $B_{\mathrm{cr}, n}^{+, 0}$ is the subsheaf of $B_{\mathrm{cr}, n}^{+}$built from sections that map to $H_{n}$ via the map $B_{\mathrm{cr}, n}^{+} \rightarrow \overline{\mathcal{O}}_{n}$.

For $r \geq 0$, there is a natural morphism

$$
\beta_{i, r}: H_{\mathrm{cr}}^{i}\left(X_{n} / W_{n}(k), J_{X_{n} / W_{n}(k)}^{[r]}\right) \rightarrow H^{i}\left(\widetilde{X}_{\text {ét }}, F^{r} B_{\mathrm{cr}, n}^{+}\right) .
$$

In fact, for any abelian sheaf $\mathcal{F}$ on the crystalline site of $X_{n}$ over $W_{n}(k)$, we have a map $R u_{*} \mathcal{F} \rightarrow R \psi_{*} \mathcal{F}\left(B_{\mathrm{cr}, n}^{+, 0}\right)$. Here, $\mathcal{F}\left(B_{\mathrm{cr}, n}^{+, 0}\right)$ is a sheaf on $\widetilde{X}_{\text {ét }}$ defined in the following way. For $\left(U, U^{\prime}\right)$ such that $B_{\text {cr, }, n}^{+, 0}$ is a PD-thickening of $H_{n}$, set $\mathcal{F}\left(B_{\mathrm{cr}, n}^{+, 0}\right)\left(U, U^{\prime}\right):=\mathcal{F}\left(B_{\mathrm{cr}, n}^{+, 0}\left(U, U^{\prime}\right)\right)$. To define the last map, simply note that we have a natural map on global sections $u_{*} \mathcal{F} \rightarrow \psi_{*} \mathcal{F}\left(B_{\mathrm{cr}, n}^{+, 0}\right)$ and the functor $\mathcal{F} \mapsto \psi_{*} \mathcal{F}\left(B_{\mathrm{cr}, n}^{+, 0}\right)$ is left exact. Taking $\mathcal{F}=J_{X_{n} / W_{n}(k)}^{[r]}$, we get the map $\beta_{i, r}$ from the composition $R u_{*} J_{X_{n} / W_{n}(k)}^{[r]} \rightarrow R \psi_{*} F^{r} B_{\mathrm{cr}, n}^{+, 0} \rightarrow R \psi_{*} F^{r} B_{\mathrm{cr}, n}^{+}$. Similarly we get a map

$$
\beta_{i, r}: H_{\mathrm{cr}}^{i}\left(X_{n} / W_{n}(k), J_{X_{n} / W_{n}(k)}^{[r]}\right) \rightarrow H^{i}\left(\widetilde{X}_{\bar{K}, \text { ét }}, F^{r} B_{\mathrm{cr}, n}^{+}\right) .
$$

The fundamental exact sequence (2.1) yields a short exact sequence of sheaves on $\widetilde{X}_{\text {ét }}$

$$
0 \rightarrow\left(\mathbf{Z} / p^{n} t^{\{r\}}\right)_{s} \rightarrow F_{p}^{r} B_{\mathrm{cr}, n}^{+} \stackrel{p^{-r} \phi-1}{\longrightarrow} B_{\mathrm{cr}, n}^{+} \rightarrow 0 .
$$

Here, for a sheaf $L$ on $\widetilde{X}_{\text {ét }}, L_{s}$ stands for its restriction to the special fiber, i.e., to the complement of the generic fiber (the site consisting of objects with trivial special fiber). For $X$ proper and $L$ torsion, proper base change theorem yields that the cohomologies of $L$ and $L_{s}$ coincide.

For $X$ proper and $r \leq p-2$, we get the long exact sequence

$$
\rightarrow H^{i}\left(\widetilde{X}_{\bar{K}, \text { ét }} \mathbf{Z} / p^{n}(r)\right) \rightarrow H^{i}\left(\widetilde{X}_{\bar{K}, \text { ét }} F^{r} B_{\mathrm{cr}, n}^{+}\right) \stackrel{\phi_{r}-1}{\longrightarrow} H^{i}\left(\widetilde{X}_{\bar{K}, \text { ét }}, B_{\mathrm{cr}, n}^{+}\right) \rightarrow H^{i+1}\left(\widetilde{X}_{\bar{K}, \text { ét }}, \mathbf{Z} / p^{n}(r)\right) \rightarrow
$$


The map $H^{i}\left(\widetilde{X}_{\bar{K} \text {,ét }}, \mathbf{Z} / p^{n}(r)\right) \rightarrow H^{i}\left(\widetilde{X}_{\bar{K} \text {,ét }}, F^{r} B_{\mathrm{cr}, n}^{+}\right)$is injective. This follows [22, Lemma 8.1] from a key theorem of Faltings [6, Th. 3.3] stating that the morphism

$$
H^{i}\left(\widetilde{X}_{\bar{K} \text {,ét }}, \mathbf{Z} / p^{n}\right) \otimes F^{r} B_{\mathrm{cr}}^{+} \rightarrow H^{i}\left(\widetilde{X}_{\bar{K} \text {,ét }}, F^{r} B_{\mathrm{cr}, n}^{+}\right)
$$

is an almost isomorphism, i.e., its kernel and cokernel are killed by a power of $m_{B}$, where $m_{B}$ is the preimage of the maximal ideal of $\bar{V}^{\wedge}$ via the map $B_{\mathrm{cr}}^{+} \rightarrow \bar{V}^{\wedge}$.

It follows that the map $\beta_{i, r}$ induces a morphism

$$
\beta_{i, r}: F^{r}\left(H_{\mathrm{cr}}^{i}\left(X_{n} / W_{n}(k)\right) \otimes B_{\mathrm{cr}, n}^{+}\right)^{\phi_{r}=1} \rightarrow H^{i}\left(\widetilde{X}_{\bar{K}, \text { ét }}, \mathbf{Z} / p^{n}(r)\right) .
$$

Composing it with the isomorphism $\rho^{*}: H^{i}\left(\widetilde{X}_{\bar{K}}\right.$,ét, $\left.\mathbf{Z} / p^{n}(r)\right) \stackrel{\sim}{\rightarrow} H^{i}\left(X_{\bar{K}}, \mathbf{Z} / p^{n}(r)\right)$ we get Faltings period morphism

$$
\alpha_{i, r}^{F}: F^{r}\left(H_{\mathrm{cr}}^{i}\left(X_{n} / W_{n}(k)\right) \otimes B_{\mathrm{cr}, n}^{+}\right)^{\phi_{r}=1} \rightarrow H^{i}\left(X_{\bar{K}}, \mathbf{Z} / p^{n}(r)\right) .
$$

Theorem 5.3. (Faltings [6, Thm. 5.3]) Let $X$ be smooth and proper over $V$, of pure relative dimension $d$. If $r+d \leq p-2$ then the period morphism $\alpha_{i, r}^{F}$ is an isomorphism.

Let now $X^{\times}$be $\log$-smooth over $V^{\times}$. As before we have sheaves $\overline{\mathcal{O}}, B_{\mathrm{cr}, n}^{+}$, and $B_{\mathrm{cr}, n}^{+, 0}$ on $\widetilde{X}_{\bar{K} \text {,ét }}$. The sheaf $B_{\mathrm{cr}, n}^{+}$is equipped with the log-structure $(\mathbf{N} \rightarrow$ $\left.B_{\mathrm{cr}, n}^{+}, 1 \mapsto[\pi]\right)$. Since Frobenius is locally surjective on $\bar{R} / p \bar{R}[7$, Lemma 3.5] $B_{\mathrm{cr}, n}^{+, 0}$ is a PD-thickening of $\mathcal{O}_{n}$ (over $R_{V, n}^{\times}$). Because of singularities it is more difficult here to relate the cohomology of $\widetilde{X}_{\bar{K}}$ to the étale cohomology of $X_{\bar{K}}$. In particular local $K(\pi, 1)$-type arguments as above do not work and one has to argue globally using Poincaré duality and compatibility with cycle classes. Faltings proves the following

Theorem 5.4. Let $X^{\times}$be a saturated, log-smooth, vertical, and proper logscheme over $V^{\times}$. Then

(1) we have an isomorphism [7, Theorem 4.9]

$$
\rho^{*}: H^{i}\left(\widetilde{X}_{\bar{K}, \text { ét }}, \mathbf{Z} / p^{n}(r)\right) \stackrel{\sim}{\rightarrow} H^{i}\left(X_{\bar{K}}, \mathbf{Z} / p^{n}(r)\right) ;
$$

(2) we have an almost isomorphism [7, Cor. 3.1]

$$
v_{i}: H^{i}\left(\widetilde{X}_{\bar{K}, \text { ét }}, \mathbf{Z} / p^{n}\right) \otimes B_{\mathrm{cr}, n}^{+} \stackrel{\sim}{\rightarrow} H^{i}\left(\widetilde{X}_{\bar{K}, \text { ét }}, B_{\mathrm{cr}, n}^{+}\right) .
$$


For $r \geq 0$, there is a natural morphism

$$
\beta_{i, r}: H_{\mathrm{cr}}^{i}\left(X_{n}^{\times} / R_{V, n}^{\times}, J_{X_{n}^{\times} / R_{V, n}^{\times}}^{[r]}\right) \rightarrow H^{i}\left(\widetilde{X}_{\bar{K}, \text { ét }}, F^{r} B_{\mathrm{cr}, n}^{+}\right)
$$

constructed just as above in the good reduction case. More generally, we can construct a morphism

$$
\beta: R \Gamma_{\mathrm{cr}}\left(X_{n}^{\times} / R_{V, n}^{\times}, \mathcal{O}_{X_{n}^{\times} / R_{V, n}^{\times}}\right) \rightarrow R \Gamma\left(\widetilde{X}_{\bar{K}, \mathrm{ét}}, B_{\mathrm{cr}, n}^{+}\right) .
$$

Theorem 5.5. (Faltings [7, Cor. 5.4]) Let $X^{\times}$be a saturated, log-smooth, vertical, and proper log-scheme over $V^{\times}$of relative dimension $d$. The almost morphism

$\alpha: R \Gamma_{\mathrm{cr}}\left(X_{n}^{\times} / R_{V, n}^{\times}, \mathcal{O}_{X_{n}^{\times} / R_{V, n}^{\times}}\right) \otimes_{R_{V, n}}^{L} B_{\mathrm{cr}, n}^{+} \rightarrow R \Gamma\left(X_{\bar{K}}, \mathbf{Z} / p^{n}\right) \otimes^{L} B_{\mathrm{cr}, n}^{+}, \quad \alpha=\rho^{*} v^{-1} \beta$, has an inverse up to $t^{d}$ (that is, composition either way is the multiplication by $\left.t^{d}\right)$. It is Galois equivariant, compatible with Frobenius and filtration.

Passing to the limit over $n$ and tensoring with $\mathbf{Q}$ in the above yields an almost morphism

$$
\alpha: R \Gamma_{\mathrm{cr}}\left(X^{\times} / R_{V}^{\times}, \mathcal{O}_{X^{\times} / R_{V}^{\times}}\right) \otimes_{R_{V}}^{L} B_{\mathrm{cr}}^{+}[1 / p] \rightarrow R \Gamma\left(X_{\bar{K}}, \mathbf{Z}_{p}\right) \otimes^{L} B_{\mathrm{cr}}^{+}[1 / p]
$$

Taking cohomology we get Faltings period isomorphism

$$
\alpha_{i, r}^{F}: H_{\mathrm{cr}}^{i}\left(X^{\times} / R_{V}^{\times}, \mathcal{O}_{X^{\times} / R_{V}^{\times}}\right) \otimes_{R_{V}[1 / p]} B_{\mathrm{cr}} \stackrel{\sim}{\rightarrow} H^{i}\left(X_{\bar{K}}, \mathbf{Q}_{p}\right) \otimes B_{\mathrm{cr}} .
$$

By looking at Frobenius invariants one can check (see $[20,2.6]$ ) that the almost morphism $\alpha_{i, r}^{F}$ is in fact an actual morphism.

5.1. Compatibility with higher Chern classes. Let us look at the integral case first. Let $X$ be a smooth and proper scheme over $W(k)$ and $0 \leq r \leq p-2$. To understand the induced isomorphism between syntomic and étale cohomology notice that we have a commutative diagram

$$
\begin{aligned}
& F^{r}\left(H_{\mathrm{cr}}^{i}\left(X_{n} / W_{n}(k)\right) \otimes B_{\mathrm{cr}, n}^{+}\right) \stackrel{\beta_{i, r}}{\longrightarrow} H^{i}\left(\widetilde{X}_{\bar{K} \text {,ét }}, F^{r} B_{\mathrm{cr}, n}^{+}\right) \\
& \downarrow \imath \text { \a.is. } \\
& H_{\mathrm{cr}}^{i}\left(X_{\bar{V}} / W_{n}(k), J_{X_{n} / W_{n}(k)}^{[r]}\right) \stackrel{\beta_{i, r}}{\longrightarrow} H^{i}\left(\left(\widetilde{X}_{\bar{V}}\right)_{\bar{K}, \text { ét }}, F^{r} B_{\mathrm{cr}, n}^{+}\right) .
\end{aligned}
$$

Here we wrote $H^{i}\left(\left(\widetilde{X}_{\bar{V}}\right)_{\bar{K} \text {,ét }}, F^{r} B_{\mathrm{cr}, n}^{+}\right)$for the limit inj $\lim _{V^{\prime} / V} H^{i}\left(\left(\widetilde{X}_{V^{\prime}}\right)_{\bar{K} \text {,ét }}, F^{r} B_{\mathrm{cr}, n}^{+}\right)$, where $V^{\prime}$ is a finite extension of $V$. The map on the right is an almost isomorphism because both terms are almost isomorphic to $H^{i}\left(X_{\bar{K}}, \mathbf{Z} / p^{n}\right) \otimes F^{r} B_{\mathrm{cr}}^{+}$. Using the 
long exact sequences (5.1), (2.1), and the above diagram we see that Faltings period isomorphism induces an isomorphism

$$
\alpha_{i, r}^{F}: H^{i}\left(X_{\bar{V}}, S_{n}(r)\right) \stackrel{\sim}{\rightarrow} H^{i}\left(X_{\bar{K}}, \mathbf{Z} / p^{n}(r)\right)
$$

as the composition

$$
\begin{aligned}
\alpha_{i, r}^{F}: H^{i}\left(X_{\bar{V}}, S_{n}(r)\right) \stackrel{\beta_{i, r}}{\rightarrow} H^{i}\left(\left(\widetilde{X}_{\bar{V}}\right)_{\bar{K}, \text { ét }}, \mathbf{Z} / p^{n}(r)_{s}\right) \\
\\
\simeq H^{i}\left(\left(\widetilde{X}_{\bar{V}}\right)_{\bar{K}, \text { ét }}, \mathbf{Z} / p^{n}(r)\right) \stackrel{\sim}{\rightarrow} H^{i}\left(X_{\bar{K}}, \mathbf{Z} / p^{n}(r)\right) .
\end{aligned}
$$

Notice that this map is already defined over any finite extension of $V$.

Theorem 5.6. Let $X$ be a smooth and proper scheme over a finite extension $V$ of $W(k)$. For $2 r \geq i+2$ we have the following commutative diagram

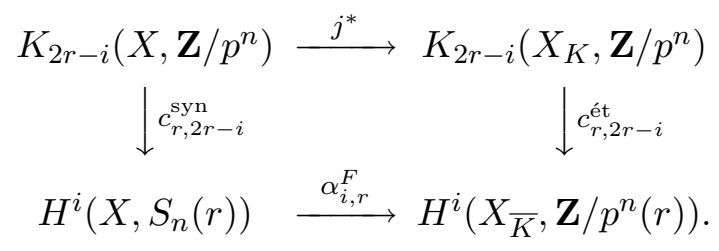

Similarly for integral $K$-theory and $2 r \geq i$.

Proof. Here the period map $\alpha_{i, r}^{F}$ is defined as the composition

$\alpha_{i, r}^{F}: H^{i}\left(X, S_{n}(r)\right) \stackrel{\beta_{i, r}}{\rightarrow} H^{i}\left(\widetilde{X}_{\bar{K}, \text { ét }}, \mathbf{Z} / p^{n}(r)_{s}\right) \stackrel{\sim}{\leftarrow} H^{i}\left(\widetilde{X}_{\bar{K}, \text { ét }}, \mathbf{Z} / p^{n}(r)\right) \stackrel{\sim}{\rightarrow} H^{i}\left(X_{\bar{K}}, \mathbf{Z} / p^{n}(r)\right)$.

First, we need a definition of Chern classes

$$
\begin{aligned}
& c_{i, j}: K_{j}\left(X, \mathbf{Z} / p^{n}\right) \rightarrow H^{2 i-j}\left(\widetilde{X}_{\text {ét }}, \mathbf{Z} / p^{n}(i)\right), \\
& c_{i, j}: K_{j}(X) \rightarrow H^{2 i-j}\left(\widetilde{X}_{\text {ét }}, \mathbf{Z} / p^{n}(i)\right) .
\end{aligned}
$$

For that set $c_{0}: \mathbf{Z} / p^{n} \rightarrow R \psi_{*} \mathbf{Z} / p^{n}$ equal to the canonical map. Set $c_{1}: \mathcal{O}_{X}^{*}[-1] \rightarrow$ $R \psi_{*} \mathbf{Z} / p^{n}(1)$ equal to the composition

$$
\mathcal{O}_{X}^{*}[-1] \rightarrow R \psi_{*} \psi^{*} \mathcal{O}_{X}^{*}[-1] \rightarrow R \psi_{*} \overline{\mathcal{O}}^{*}[-1] \rightarrow R \psi_{*} \mathbf{Z} / p^{n}(1),
$$

where the last map is induced by the Kummer exact sequence

$$
0 \rightarrow \mathbf{Z} / p^{n}(1) \rightarrow \overline{\mathcal{O}}^{*} \stackrel{p^{n}}{\longrightarrow} \overline{\mathcal{O}}^{*} \rightarrow 0
$$

These maps are clearly compatible (via the map $\rho^{*}$ ) with the maps $c_{0}$ and $c_{1}$ in the étale cohomology of $X_{K}$. We have a projective space theorem 
Proposition 5.7. Let $m>0, \xi=c_{1}(\mathcal{O}(1)) \in H^{2}\left(\widetilde{\mathbf{P}_{X \text { ét }}^{m}}, \mathbf{Z} / p^{n}(1)\right)$. Let $\pi_{X}$ be the projection $\mathbf{P}_{X}^{m} \rightarrow X$, then the natural map

$$
\oplus_{i} \xi_{1}^{i} \cup \pi_{X}^{*}: \bigoplus_{i=0}^{m} H^{l-2 i}\left(\widetilde{X}_{\text {ét }}, \mathbf{Z} / p^{n}(r-i)\right) \rightarrow H^{l}\left(\widetilde{\mathbf{P}_{X}^{m}}, \mathbf{Z} / p^{n}(r)\right)
$$

is an isomorphism for all $r$.

Proof. Reduce to the projective space theorem in étale cohomology.

Like in $[13,2.2]$ we can now construct universal classes $C_{i} \in H^{2 i}(\widetilde{B . G L} / W(k)$ ét $\left.\mathbf{Z} / p^{n}(i)\right)$ that are compatible with the étale universal classes $C_{i}^{\text {ét }} \in H^{2 i}(B . G L / K$, $\left.\mathbf{Z} / p^{n}(i)\right)$. We claim that they are also compatible with the syntomic universal classes $C_{i}^{\text {syn }} \in H^{2 i}\left(B . G L / W(k), S_{n}(i)\right)$, for $i \leq p-2$ (via the map $\beta_{*}$ ). Consider the following commutative diagram

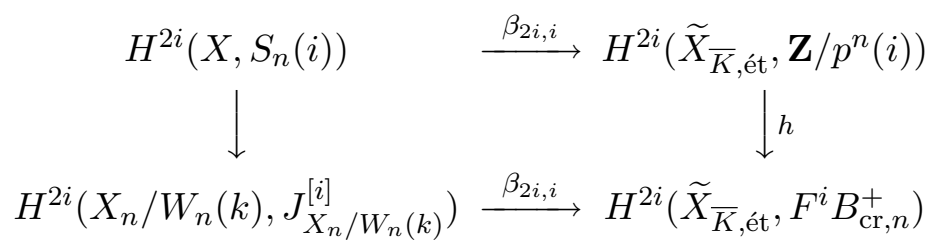

As we have mentioned before the right vertical map is an injection. By compatibility of syntomic and crystalline Chern classes it suffices to show the compatibility of the crystalline universal classes $C_{i}^{\text {cr }}$ with the universal classes $C_{i}$ (via the maps $\beta_{2 i, i}$ and $\left.h\right)$. The classes $C_{i}^{\mathrm{cr}} \in H^{2 i}\left(B . G L / W_{n}(k), J^{[i]}\right)$ are pullbacks of classes $C_{i, m}^{\mathrm{cr}} \in H^{2 i}\left(B . G L_{m} / W_{n}(k), J^{[i]}\right)$, for $i \leq m$, and those can be defined using the projective space theorem in crystalline cohomology and the crystalline Chern classes

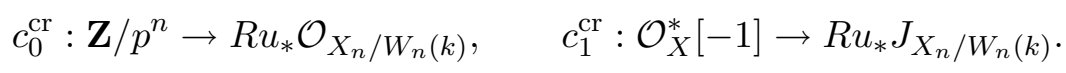

We know that the morphism $\beta_{i}$ is compatible with products. Faltings checked $[7$, Theorem 5.2] that it is also compatible with the Chern classes $c_{0}, c_{1}$ in crystalline and $\widetilde{X}_{\bar{K}}$-cohomology. Comparing the two projective space theorems yields now that $\beta_{2 i, i}\left(C_{i, m}^{\mathrm{cr}}\right)=h\left(C_{i, m}\right)$, for $i \leq m$. By construction of higher Chern classes this suffices to prove our theorem.

Let us look now at the rational semistable case. Recalling how we pass from Faltings period isomorphism to the one of Fontaine-Jannsen and how we then pass to a morphism from syntomic cohomology to étale cohomology (Lemma 
2.5), and inspecting the large diagram in section 1.4, we see that Faltings period map

$$
\alpha_{i, r}: H_{\mathrm{cr}}^{i}\left(X_{n}^{\times} / R_{V, n}^{\times}, \mathcal{O}_{X_{n}^{\times} / R_{V, n}^{\times}}\right) \otimes_{R_{V, n}} B_{\mathrm{cr}, n}^{+} \rightarrow H^{i}\left(X_{\bar{K}}, \mathbf{Z} / p^{n}\right) \otimes B_{\mathrm{cr}, n}^{+}
$$

induces a map on syntomic cohomology via the composition

$$
\begin{aligned}
& H^{i}\left(X_{\bar{V}}^{\times} / W(k)\right)[1 / p] \stackrel{\sim}{\rightarrow}\left(H^{i}\left(X_{\bar{V}}^{\times} / R_{V}^{\times}\right)[1 / p]\right)^{N=0} \underset{\leftarrow}{\leftarrow}\left(H_{\mathrm{cr}}^{i}\left(X^{\times} / R_{V}^{\times}\right) \otimes_{R_{V}} \widehat{B}_{\mathrm{st}}^{+}[1 / p]\right)^{N=0} \\
& \stackrel{\omega}{\simeq} H_{\mathrm{cr}}^{i}\left(X^{\times} / R_{V}^{\times}\right) \otimes_{R_{V}} B_{\mathrm{cr}}^{+}[1 / p] \stackrel{\alpha_{i, r}^{F}}{\rightarrow} H^{i}\left(X_{\bar{K}}, \mathbf{Q}_{p}\right) \otimes B_{\mathrm{cr}}^{+}[1 / p] .
\end{aligned}
$$

Set $\widetilde{X}_{\bar{V} \text {,ét }}=\left(\widetilde{X}_{\bar{V}}\right)_{\bar{K} \text {,ét }}$. We have the commutative diagram

$$
\begin{aligned}
& H^{i}\left(X_{\bar{V}, n}^{\times} / W_{n}(k)\right) \longrightarrow H^{i}\left(X_{\bar{V}, n}^{\times} / R_{V, n}^{\times}\right) \stackrel{\sim}{\longleftarrow} H_{\mathrm{cr}}^{i}\left(X_{n}^{\times} / R_{V, n}^{\times}\right) \otimes_{R_{V, n}} \widehat{B}_{\mathrm{st}, n}^{+} \\
& H^{i}\left(\widetilde{X}_{\bar{V}, \text { ét }}, F^{r} B_{\mathrm{cr}, n}^{+}\right)=H^{i}\left(\widetilde{X}_{\bar{V}, \text { ét }}, F^{r} B_{\mathrm{cr}, n}^{+}\right)=H^{i}\left(\widetilde{X}_{\bar{V}, \text { ét }}, F^{r} B_{\mathrm{cr}, n}^{+}\right) \\
& H_{\mathrm{cr}}^{i}\left(X_{n}^{\times} / R_{V, n}^{\times}\right) \otimes_{R_{V, n}} \widehat{B}_{\mathrm{st}, n}^{+} \stackrel{p_{1}}{\longrightarrow} H_{\mathrm{cr}}^{i}\left(X_{n}^{\times} / R_{V, n}^{\times}\right) \otimes_{R_{V, n}} B_{\mathrm{cr}, n}^{+} \\
& \downarrow \beta_{i} \otimes \beta_{0} \quad \downarrow \beta_{i} \otimes \mathrm{Id} \\
& H^{i}\left(\widetilde{X}_{\bar{V}, \text { ét }}, F^{r} B_{\mathrm{cr}, n}^{+}\right) \quad=\quad H^{i}\left(\widetilde{X}_{\bar{V}, \text { ét }}, F^{r} B_{\mathrm{cr}, n}^{+}\right)
\end{aligned}
$$

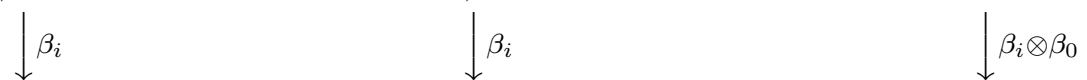

All the maps $\beta_{*}$ are defined as above. The map $\beta_{0}$ factors as the composition of the projection $p_{1}: \widehat{B}_{\mathrm{st}, n}^{+} \rightarrow B_{\mathrm{cr}, n}^{+}$and the natural map $B_{\mathrm{cr}, n}^{+} \rightarrow H^{i}\left(\widetilde{X}_{\bar{V} \text {,ét }}, F^{r} B_{\mathrm{cr}, n}^{+}\right)$. This is so because $\widehat{B}_{\mathrm{st}, n}^{+}$is the final object in the crystalline site of $\bar{V}_{n}^{\times}$over $R_{V, n}^{\times}$. Notice that from the definition of the map $\omega$ it follows that $p_{1}$ is the inverse of

$$
\omega: H_{\mathrm{cr}}^{i}\left(X^{\times} / R_{V}^{\times}\right) \otimes_{R_{V}} B_{\mathrm{cr}}^{+}[1 / p] \stackrel{\sim}{\rightarrow}\left(H_{\mathrm{cr}}^{i}\left(X^{\times} / R_{V}^{\times}\right) \otimes_{R_{V}} \widehat{B}_{\mathrm{st}}^{+}[1 / p]\right)^{N=0}
$$

We computed that Faltings period morphism induces the map

$$
\beta_{i, r}: H^{i}\left(X_{\bar{V}, n}^{\times} / W_{n}(k), J_{X_{\bar{V}, n}^{\times} / W_{n}(k)}^{[r]}\right) \rightarrow H^{i}\left(\widetilde{X}_{\bar{V}, \mathrm{ét}}, F^{r} B_{\mathrm{cr}, n}^{+}\right) .
$$

Since all the maps in the above diagram are compatible with filtration and Frobenius, we obtain a canonical map

$$
\beta_{i, r}: H^{i}\left(X^{\times}, S_{n}^{\prime}(r)\right) \rightarrow H^{i}\left(X_{\bar{K}}, \mathbf{Z} / p^{n}(r)_{s}^{\prime}\right) .
$$

More precisely, we get a canonical map from $H^{i}\left(X^{\times}, S_{n}^{\prime}(r)\right)$ to the $\widetilde{X}_{\bar{K}^{-}}$-cohomology of the cone of $\phi-p^{r}: F^{r} B_{\mathrm{cr}, n}^{+} \rightarrow B_{\mathrm{cr}, n}^{+}$, which in turn maps via multiplication by $p^{r}$ on $F^{r} B_{\mathrm{cr}, n}^{+}$to the $\widetilde{X}_{\bar{K}^{-}}$cohomology of the cone of $\phi_{r}-1: F_{p}^{r} B_{\mathrm{cr}, n}^{+} \rightarrow B_{\mathrm{cr}, n}^{+}$. 
But the last cone by the fundamental exact sequence (2.1) is quasi-isomorphic to $\mathbf{Z} / p^{n}(r)_{s}^{\prime}$. Hence Faltings period isomorphism induces a morphism

$$
\beta_{i, r}: H^{i}\left(X_{\bar{V}}^{\times}, S_{n}^{\prime}(r)\right) \rightarrow H^{i}\left(X_{\bar{K}}, \mathbf{Z} / p^{n}(r)^{\prime}\right)
$$

as the composition

$\beta_{i, r}: H^{i}\left(X_{\bar{V}}^{\times}, S_{n}(r)\right) \stackrel{\beta_{i, r}}{\rightarrow} H^{i}\left(\widetilde{X}_{\bar{V}, \text { ét }}, \mathbf{Z} / p^{n}(r)_{s}^{\prime}\right) \stackrel{\sim}{\leftarrow} H^{i}\left(\widetilde{X}_{\bar{V} \text {,ét }}, \mathbf{Z} / p^{n}(r)^{\prime}\right) \stackrel{\sim}{\rightarrow} H^{i}\left(X_{\bar{K}}, \mathbf{Z} / p^{n}(r)^{\prime}\right)$.

It follows that the induced morphism $\alpha_{i, r}^{F}: H^{i}\left(X_{\bar{V}}^{\times}, S_{\mathbf{Q}_{p}}^{\prime}(r)\right) \rightarrow H^{i}\left(X_{\bar{K}}, \mathbf{Q}_{p}(r)\right)$ is equal to the composition

$$
H^{i}\left(X_{\bar{V}}^{\times}, S_{\mathbf{Q}_{p}}^{\prime}(r)\right) \stackrel{\alpha_{i, r}^{F}}{\rightarrow} H^{i}\left(X_{\bar{K}}, \mathbf{Q}_{p}(r)\right) \stackrel{p^{-r}}{\rightarrow} H^{i}\left(X_{\bar{K}}, \mathbf{Q}_{p}(r)\right) .
$$

Theorem 5.8. Let $X^{\times}$be saturated, log-smooth, vertical and proper over $V^{\times}$. For $2 r \geq i+2$ we have the following commutative diagram

$$
\begin{array}{rrr}
K_{2 r-i}\left(X, \mathbf{Z} / p^{n}\right) & j^{*} & K_{2 r-i}\left(X_{K}, \mathbf{Z} / p^{n}\right) \\
\downarrow c_{r, 2 r-i}^{\mathrm{syn}} & & \\
H^{i}\left(X^{\times}, S_{n}^{\prime}(r)\right) \stackrel{\beta^{r} c_{r, 2 r-i}^{\text {ét }}}{\longrightarrow} & H^{i}\left(X_{\bar{K}}, \mathbf{Z} / p^{n}(r)^{\prime}\right) .
\end{array}
$$

Similarly for integral $K$-theory and $2 r \geq i$.

Proof. The period morphism $\beta_{i, r}$ is defined as the composition

$$
H^{i}\left(X^{\times}, S_{n}^{\prime}(r)\right) \stackrel{\beta_{i, r}}{\rightarrow} H^{i}\left(\widetilde{X}_{\bar{K}, \text { ét }}, \mathbf{Z} / p^{n}(r)_{s}^{\prime}\right) \stackrel{\sim}{\leftarrow} H^{i}\left(\widetilde{X}_{\bar{K}, \text { ét }}, \mathbf{Z} / p^{n}(r)^{\prime}\right) \stackrel{\sim}{\rightarrow} H^{i}\left(X_{\bar{K}}, \mathbf{Z} / p^{n}(r)^{\prime}\right) .
$$

Arguing as in the proof of Theorem 5.6, we get Chern classes into the cohomology $H^{i}\left(\widetilde{X}_{\bar{K} \text {,ét }}, \mathbf{Z} / p^{n}(r)^{\prime}\right)$ that are compatible with the étale Chern classes. We need to show that they are also compatible with the syntomic Chern classes. For that it is enough to look at the universal classes. We have the universal classes $C_{i} \in H^{2 i}\left(\widetilde{B . G L} / W(k)_{\text {ét }}, \mathbf{Z} / p^{n}(i)\right)$ that are compatible with the étale universal classes $C_{i}^{\text {ét }} \in H^{2 i}\left(B . G L / K, \mathbf{Z} / p^{n}(i)\right)$. We claim that the classes $p^{i} C_{i}$ are also compatible with the syntomic universal classes $C_{i}^{\text {syn }} \in H^{2 i}\left(B . G L / W(k), S_{n}^{\prime}(i)\right)$ (via the map $\beta_{*}$ ). Consider the following commutative diagram

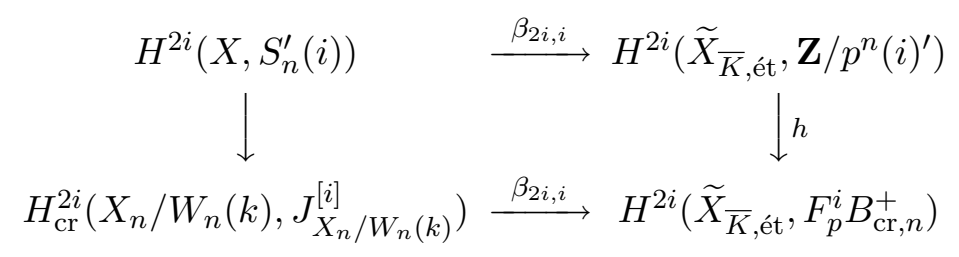


As before the right vertical map is an injection. By compatibility of syntomic and crystalline Chern classes it suffices to show the compatibility of the crystalline universal classes $C_{i}^{\mathrm{cr}}$ with the universal classes $p^{i} C_{i}$ via the maps $\beta_{2 i, i}$ and $h$. Untwisting multiplication by $p^{i}$ from the definition of $\beta_{2 i, i}$ we see that we need to show that the classes $C_{i}^{\mathrm{cr}}$ and $C_{i}$ map to the same class in $H^{2 i}\left((\widetilde{B \cdot G L} / W(k))_{\bar{K} \text {,ét }}, F^{i} B_{\mathrm{cr}, n}^{+}\right)$. The classes $C_{i}^{\text {cr }} \in H^{2 i}\left(B . G L / W_{n}(k), J^{[i]}\right)$ are pullbacks of classes $C_{i, m}^{\mathrm{cr}} \in H^{2 i}(B . G$ $\left.L_{m} / W_{n}(k), J^{[i]}\right)$, for $i \leq m$, and those can be defined using the projective space theorem in filtered crystalline cohomology. From here we argue exactly as in the proof of Theorem 5.6.

The following corollary follows immediately from that and Lemma 2.7.

Corollary 5.9. Let $X^{\times}$be saturated, log-smooth, vertical and proper over $V^{\times}$. For $2 r \geq i+2$ we have the following commutative diagram

$$
\begin{aligned}
& \mathbf{Q}_{p} \otimes \operatorname{proj} \lim _{n} K_{2 r-i}\left(X_{\bar{V}}^{\times}, \mathbf{Z} / p^{n}\right) \stackrel{j^{*}}{\longrightarrow} \mathbf{Q}_{p} \otimes \operatorname{proj} \lim _{n} K_{2 r-i}\left(X_{\bar{K}}, \mathbf{Z} / p^{n}\right) \\
& \downarrow c_{r, 2 r-i}^{\mathrm{syn}} \quad \downarrow c_{r, 2 r-i}^{e^{e t}} \\
& H^{i}\left(X_{\bar{V}}^{\times}, \mathcal{S}_{\mathbf{Q}_{p}}^{\prime}(r)\right) \quad \stackrel{\alpha_{i, r}^{F}}{\longrightarrow} \quad H^{i}\left(X_{\bar{K}}, \mathbf{Q}_{p}(r)\right) .
\end{aligned}
$$

Similarly for integral $K$-theory and $2 r \geq i$.

Let now $X$ be a smooth and proper scheme over $V$. The above gives us Faltings rational crystalline period isomorphism

$$
\alpha_{i}^{F}: H_{\mathrm{cr}}^{i}\left(X_{0} / W(k)\right) \otimes B_{\mathrm{cr}} \stackrel{\sim}{\rightarrow} H^{i}\left(X_{\bar{K}}, \mathbf{Q}_{p}\right) \otimes B_{\mathrm{cr}}
$$

as the composition

$$
\begin{aligned}
H_{\mathrm{cr}}^{i}\left(X_{0} / W(k)\right) \otimes B_{\mathrm{cr}} & \stackrel{\sim}{\rightarrow} H_{\mathrm{cr}}^{i}\left(X_{0}^{\times} / W(k)^{0}\right) \otimes B_{\mathrm{cr}} \simeq H_{\mathrm{cr}}^{i}\left(X^{\times} / R_{V}^{\times}\right) \otimes_{R_{V}^{\times}} B_{\mathrm{cr}} \\
& \stackrel{\alpha_{i}^{F}}{\simeq} H^{i}\left(X_{\bar{K}}, \mathbf{Q}_{p}\right) \otimes B_{\mathrm{cr}} .
\end{aligned}
$$

It induces the following map on syntomic cohomology

$$
\alpha_{i, r}^{F}: \quad H^{i}\left(X_{\bar{V}}, \mathcal{S}_{\mathbf{Q}_{p}}^{\prime}(r)\right) \rightarrow H^{i}\left(X_{\bar{V}}^{\times}, \mathcal{S}_{\mathbf{Q}_{p}}^{\prime}(r)\right) \stackrel{\alpha_{i, r}^{F}}{\rightarrow} H^{i}\left(X_{\bar{K}}, \mathbf{Q}_{p}(r)\right) .
$$


Corollary 5.10. For $2 r \geq i+2$ we have the following commutative diagram

$$
\begin{aligned}
& \mathbf{Q}_{p} \otimes \operatorname{proj} \lim _{n} K_{2 r-i}\left(X_{\bar{V}}, \mathbf{Z} / p^{n}\right) \stackrel{j^{*}}{\longrightarrow} \mathbf{Q}_{p} \otimes \operatorname{proj} \lim _{n} K_{2 r-i}\left(X_{\bar{K}}, \mathbf{Z} / p^{n}\right) \\
& \downarrow c_{r, 2 r-i}^{\mathrm{syn}} \quad \downarrow c_{r, 2 r-i}^{\text {et }} \\
& H^{i}\left(X_{\bar{V}}, \mathcal{S}_{\mathbf{Q}_{p}}^{\prime}(r)\right) \quad \stackrel{\alpha_{i, r}^{F}}{\longrightarrow} \quad H^{i}\left(X_{\bar{K}}, \mathbf{Q}_{p}(r)\right) .
\end{aligned}
$$

Similarly for integral $K$-theory and $2 r \geq i$.

Proof. Since $K_{2 r-i}\left(X_{\bar{V}}, \mathbf{Z} / p^{n}\right) \stackrel{\sim}{\rightarrow} K_{2 r-i}\left(X_{\bar{V}}^{\times}, \mathbf{Z} / p^{n}\right)$ in this case and log-Chern classes are defined via the classical ones this is just a restatement of the above corollary.

\section{COMPARISON OF $p$-ADIC PERIOD MORPHISMS}

We conclude with the promised comparison of described here $p$-adic period morphisms. Assume first that $X$ is smooth and proper over $W(k)$. Let $d$ denote the dimension of $X_{K}$.

Corollary 6.1. Let $p-2 \geq \max \{r, d+2 r-i\}, 2 r-i \geq \max \{2 d+1,2\}$, and $r \geq i$. Then the integral crystalline period morphisms $\alpha_{i, r}^{K}, \alpha_{i, r}^{F}$, and $\alpha_{i, r}^{N}$

$$
\alpha_{i, r}^{*}: \quad H^{i}\left(X_{\bar{K}}, \mathbf{Z} / p^{n}(r)\right) \simeq F^{r}\left(H_{\mathrm{cr}}^{i}(X / W(k)) \otimes B_{\mathrm{cr}, n}^{+}\right)^{\phi_{r}=1}
$$

are equal.

Proof. Apply the uniqueness criterium from Theorem 3.4. The needed compatibility of the period morphism with higher Chern classes is clear in the case of the map $\alpha_{i, r}^{N}$ and was proved in Theorem 4.7 and Theorem 5.6 for the other two maps.

Let now $X$ be smooth and proper over a finite extension $V$ of $W(k)$.

Corollary 6.2. The rational crystalline period morphisms $\alpha_{i, 0}^{T}, \alpha_{i, 0}^{F}$, and $\alpha_{i, 0}^{N}$

$$
\alpha_{i, 0}^{*}: \quad H^{i}\left(X_{\bar{K}}, \mathbf{Q}_{p}\right) \otimes B_{\mathrm{cr}} \simeq H_{\mathrm{cr}}^{i}\left(X_{0} / W(k)\right) \otimes B_{\mathrm{cr}}
$$

are equal. 
Proof. Take $r$ such that $2 r-i \geq \max \{2 d+1,2\}, 2 r-i \geq 3$ for $p=2, d=0$, and $r \geq i$. It suffices to show that the crystalline period morphisms $\alpha_{i, r}^{T}, \alpha_{i, r}^{F}$, and $\alpha_{i, r}^{N}$

$$
\alpha_{i, r}^{*}: \quad H^{i}\left(X_{\bar{K}}, \mathbf{Q}_{p}(r)\right) \otimes B_{\mathrm{cr}} \simeq H_{\mathrm{cr}}^{i}\left(X_{0} / W(k)\right) \otimes B_{\mathrm{cr}}\{-r\}
$$

are equal. For that apply the uniqueness criterium from Theorem 3.8. The needed compatibility of the period morphism with higher Chern classes is clear in the case of the map $\alpha_{i, r}^{N}$ and was proved in Theorem 4.3 and Corollary 5.10 for the other two maps.

Let $X^{\times}$be a fine and saturated, log-smooth scheme over $V^{\times}$with Cartier type reduction.

Corollary 6.3. The period morphisms $\alpha_{i, 0}^{T}, \alpha_{i, 0}^{F}$, and $\alpha_{i, 0}^{N}$

$$
\alpha_{i, 0}^{*}: \quad H^{i}\left(X_{\bar{K}}, \mathbf{Q}_{p}\right) \otimes B_{\mathrm{st}} \simeq H_{\mathrm{cr}}^{i}\left(X_{0}^{\times} / W(k)^{0}\right) \otimes B_{\mathrm{st}}
$$

are equal.

Proof. Choose $r$ such that $2 r-i \geq \max \{2 d+1,2\}, 2 r-i \geq 3$ for $p=2, d=0$, and $r \geq i$. It suffices to show that the period morphisms $\alpha_{i, r}^{T}, \alpha_{i, r}^{F}$, and $\alpha_{i, r}^{N}$

$$
\alpha_{i, r}^{*}: \quad H^{i}\left(X_{\bar{K}}, \mathbf{Q}_{p}(r)\right) \otimes B_{\mathrm{st}} \simeq H_{\mathrm{cr}}^{i}\left(X_{0}^{\times} / W(k)^{0}\right) \otimes B_{\mathrm{st}}\{-r\}
$$

are equal. For that apply the uniqueness criterium from Theorem 3.11. The needed compatibility of the period morphism with higher Chern classes is clear in the case of the map $\alpha_{i, r}^{N}$ and was proved in Corollary 4.6 and Corollary 5.9 for the other two maps.

\section{REFERENCES}

[1] S. Bloch, Algebraic cycles and higher K-theory, Adv. in Math. 61 (1986), no. 3, 267-304.

[2] Ch. Breuil, Représentations p-adiques semi-stables et transversalité de Griffiths. [Semistable $p$-adic representations and Griffiths transversality] Math. Ann. 307 (1997), no. 2, 191-224.

[3] Ch. Breuil, Cohomologie étale de p-torsion et cohomologie cristalline en réduction semistable, Duke Math. J. 95 (1998), no. 3, 523-620.

[4] Ch. Breuil, W. Messing, Torsion étale and crystalline cohomologies, Cohomologies padiques et applications arithmétiques, II. Astérisque No. 279 (2002), 81-124.

[5] G. Faltings, p-adic Hodge theory, J. Amer. Math. Soc. 1 (1988), no. 1, 255-299. 
[6] G. Faltings, Crystalline cohomology and p-adic Galois representations, Algebraic analysis, geometry and number theory (J. I. Igusa ed.), Johns Hopkins University Press, Baltimore, 1989, 25-80.

[7] G. Faltings, Almost étale extensions, Cohomologies p-adiques et applications arithmétiques, II. Astérisque No. 279 (2002), 185-270.

[8] J.-M. Fontaine, Sur certains types de représentations p-adiques du groupe de Galois d'un corps local, construction d'un anneau de Barsotti-Tate, Ann. of Math. 115 (1982), 529577.

[9] J.-M. Fontaine, Le corp des périodes p-adiques, Astérisque 223, Soc. Math. de France, 1994, 59-111.

[10] J.-M. Fontaine and G. Lafaille, Construction de représentations p-adiques, Ann. Sci. Ec. Norm. Sup., IV. Ser., 15 (1982), 547-608.

[11] J.-M. Fontaine and W. Messing, p-adic periods and p-adic étale cohomology, Current Trends in Arithmetical Algebraic Geometry (K. Ribet, ed.), Contemporary Math., vol. 67, Amer. Math. Soc., Providence, 1987, 179-207.

[12] T. Geisser, M. Levine, The K-theory of fields in characteristic $p$, Invent. Math. 139 (2000), no. 3, 459-493.

[13] H. Gillet, Riemann-Roch theorems for higher algebraic K-theory, Adv. Math. 40 (1981), $203-289$.

[14] M. Gros, Régulateurs syntomiques et valeurs de fonctions L p-adiques I, Invent. Math. 99 (1990), 293-320.

[15] O. Hyodo, K. Kato, Semistable reduction and crystalline cohomology with logarithmic poles, Astérisque 223, Soc. Math. de France, 1994, 221-268.

[16] K. Kato, On p-adic vanishing cycles (application of ideas of Fontaine-Messing), Algebraic Geometry, Sendai, 1985, Adv. Stud. Pure Math. 10, North-Holland, Amsterdam-New York, 1987, 207-251.

[17] K. Kato, Logarithmic structures of Fontaine-Illusie, Algebraic analysis, geometry, and number theory (Baltimore, MD, 1988), 191-224, Johns Hopkins Univ. Press, Baltimore, MD, 1989.

[18] K. Kato, Semistable reduction and p-adic étale cohomology, Astérisque 223, Soc. Math. de France, 1994, 269-293.

[19] K. Kato, W. Messing, Syntomic cohomology and p-adic étale cohomology, Tôhoku Math. J. 44 (1992), 1-9.

[20] M. Kisin, Potential semi-stability of p-adic étale cohomology, Israel J. Math. 129 (2002), 157-173.

[21] M. Kurihara, A note on p-adic étale cohomology, Proc. Japan Acad. Ser. A Math. Sci. 63 (1987), no. 7, 275-278.

[22] W. Niziol, Duality in the cohomology of crystalline local systems, Compositio Math. 109 (1997), no. 1, 67-97.

[23] W. Nizioł, Crystalline Conjecture via K-theory, Ann. Sci. École Norm. Sup. (4) 31 (1998), no. $5,659-681$. 
[24] W. Niziol, Toric singularities: log-blow-ups and global resolutions, J. Algebraic Geom. 15 (2006), 1-29.

[25] W. Nizioł, Semistable Conjecture via K-theory, Duke Math. J. 141 (2008), no. 1, 151-178.

[26] W. Niziol, p-adic motivic cohomology and arithmetic, International Congress of Mathematicians. Vol. II, 459-472, Eur. Math. Soc., Zürich, 2006.

[27] T. Saito, Log smooth extension of a family of curves and semi-stable reduction. J. Algebraic Geom. 13 (2004), no. 2, 287-321.

[28] C. Soulé, Operations on étale K-theory. Applications., Algebraic K-theory I, Lect. Notes Math. 966, Springer-Verlag, Berlin, Heidelberg and New York, 1982, 271-303.

[29] A. Suslin, Higher Chow groups and étale cohomology, Cycles, transfers, and motivic homology theories, 239-254, Ann. of Math. Stud., 143, Princeton Univ. Press, Princeton, NJ, 2000 .

[30] T. Tsuji, p-adic Hodge theory in the semi-stable reduction case, Proceedings of the International Congress of Mathematicians, Vol. II (Berlin, 1998). Doc. Math. 1998, Extra Vol. II, 207-216 (electronic).

[31] T. Tsuji, p-adic étale and crystalline cohomology in the semistable reduction case, Invent. Math. 137 (1999), no. 2, 233-411.

[32] T. Tsuji, On p-adic nearby cycles of log smooth families, Bull. Soc. Math. France 128 (2000), no. 4, 529-575.

[33] V. Voevodsky, Motivic cohomology with Z/2-coefficients, Publ. Math. Inst. Hautes Études Sci. No. 98 (2003), 59-104.

[34] V. Voevodsky, On motivic cohomology with Z/l-coefficients, preprint, 2003.

Wieslawa Niziol Department of Mathematics

College of Science, University of Utah, Salt Lake City

E-mail: niziol@math.utah.edu 